UNIVERSIDADE DE BRASÍLIA - FACULDADE DE CEILÂDIA PROGRAMA DE PÓS-GRADUAÇÃO STRICTU-SENSU EM CIÊNCIAS E TECNOLOGIAS EM SAÚDE

EFEITOS DO TRATAMENTO MANIPULATIVO OSTEOPÁTICO NA FUNÇÃO CARDIOVASCULAR EM INDIVÍDUOS SAUDÁVEIS E COM INSUFICIÊNCIA CARDÍACA

FELLIPE AMATUZZI TEIXEIRA

ORIENTADOR: Prof. Dr. GERSON CIPRIANO JUNIOR 
UNIVERSIDADE DE BRASÍLIA - FACULDADE DE CEILÂDIA PROGRAMA DE PÓS-GRADUAÇÃO STRICTO-SENSU EM CIÊNCIAS E TECNOLOGIAS EM SAÚDE

\section{EFEITOS DO TRATAMENTO MANIPULATIVO OSTEOPÁTICO NA FUNÇÃO CARDIOVASCULAR EM INDIVÍDUOS SAUDÁVEIS E COM INSUFICIÊNCIA CARDÍACA}

FELLIPE AMATUZZI TEIXEIRA

Tese apresentada à Faculdade da
Ceilândia da Universidade de Brasília
como requisito parcial para obtenção do
título de Doutor em Ciências e
Tecnologias em Saúde

ORIENTADOR: Prof. Dr. GERSON CIPRIANO JUNIOR

BRASÍLIA, 2015 
UNIVERSIDADE DE BRASÍLIA

FACULDADE DE CEILÂNDIA

PROGRAMA DE PÓS-GRADUAÇÃO EM CIÊNCIAS E TECNOLOGIAS EM SAÚDE

\section{EFEITOS DO TRATAMENTO MANIPULATIVO OSTEOPÁTICO NA FUNÇÃO CARDIOVASCULAR EM INDIVÍDUOS SAUDÁVEIS E COM INSUFICIÊNCIA CARDÍACA}

\section{FELLIPE AMATUZZI TEIXEIRA}

Área de concentração: Promoção, prevenção e intervenção em saúde.

Linha de pesquisa: saúde, funcionalidade, ocupação e trabalho.

Tese de doutorado submetida ao programa de Pós-Graduação em Ciências e Tecnologias da Saúde da Universidade de Brasília como parte dos requisitos necessários para a obtenção do grau de doutor.

APROVADA POR:

GERSON CIPRIANO JÚNIOR (UnB) - Orientador

GUILHERME ECKHARDT MOLINA (UnB) - Examinador externo 1

GUSTAVO DE AZEVEDO CARVALHO (UCB) - Examinador externo 2

WAGNER RODRIGUES MARTINS (UnB) - Examinador externo 3

JOÃO PAULO CHIEREGATO MATHEUS (UnB) - Examinador interno

DATA: BRASÍLIA/DF: 01 DE DEZEMBRO DE 2015 


\section{DEDICATÓRIA}

O doutorado é um curso em que podemos exercer uma contribuição a ciência. Ter como inspiração alguns dos pesquisadores mais lembrados pela história da osteopatia tal como o Denslow, $\mathrm{DO}, \mathrm{PhD}$, que pesquisava a fisiologia humana e tentava explicar por meio da luz da ciência o que acontecia aos pacientes que sofriam intervenção osteopática é de fato algo que me fez seguir adiante com as idéias apresentadas nessa tese. Osteopatas clínicos que fazem o seu trabalho no dia a dia tentando amenizar o sofrimento das pessoas também são importantes como inspiração desse trabalho. Paulo Roberto Dobruski (Curitiba - PR), Carlos Augusto Pimpão Valente (Brasília - DF) e Luis Rivas Cano (Espanha) são os nomes que escolhi para representar todos esses profissionais que são a via final do nosso trabalho em ciência. As pessoas que idealizaram uma escola de osteopatia que deveria espalhar a filosofia e ciência osteopática pelo mundo e, com esse pensamento expansionista, possibilitou a chegada desse ensino ao Brasil, professores Gines Almazán e François Ricard, idealizadores da Escola de Osteopatia de Madrid e o professor Rogério Augusto Queiroz que ajudou de forma exponencial o crescimento da osteopatia no Brasil. Aos meus queridos professores de osteopatia que da sua forma fizeram com que a minha curiosidade de cientista pudesse voltar os olhos para os fenômenos osteopáticos: Rogério Queiroz, Paulo Dobruski, André Pêgas, Luis Rivas Cano, Juan José Boscá Gandiá, Pablo Escribá, Pablo Lanes, Rômulo Ordini, François Ricard, Luis Palomeque, Elena Martinez, Carlos Galcerán, Ana Paula Cardoso de Mello e Mello Ribeiro meu muito obrigado pela inspiração. Aos meus amigos feito ao longo dos anos de osteopatia. Andréa Laporta (in memorian), Vitor Hugo Martins, Leonardo Nascimento, Fernando Pucciarelli, Pedro Vidinha, Douglas Lima, Raphael Milagres que representam todas as amizades osteopáticas construídas. Um agradecimento especial ao Velho Doutor (Old Doc - Andrew Taylor Still), sem ele, nada disso estaria acontecendo. Aos meus pais pela oportunidade de estudar e incentivo a continuar estudando.

Mas esse doutorado é dedicado de fato a minha família. Minha esposa Raquel Henriques Jácomo que é a minha inspiração maior e a minha filha Júlia Jácomo Teixeira meu amor incondicional a vocês que me apoiam, me entendem e me amam como ninguém. A minha eterna gratidão a vocês meus amores. 


\section{AGRADECIMENTOS}

Agradeço em primeiro lugar a minha família, Raquel Henriques Jácomo e Júlia Jácomo Teixeira. Sem vocês nada na minha vida teria tanta doçura e amor. Amo vocês. Minha eterna gratidão.

Agradeço aos meus pais pela dedicação, por ensinar um caminho e me dar liberdade de escolha. Pude ser a pessoa que sou hoje por vocês me ensinarem como andar, me darem liberdade de ser quem eu sou. Meu eterno carinho e amor a vocês.

Aos meus grandes amigos do grupo de pesquisa GPRC: Vinícius Maldaner, Laura Neves, Luciana Vieira Tavernard, Marianne Lucena, Alexandra Lima, Fabíola, Sérgio Thomaz, Priscila Flávia, Cláudio Nakata, Sérgio Ramalho, Robson Borges, Luis Melo e todos os alunos de graduação que acompanharam todo esse processo: Isabelle Barreira, Ana Paula Xavier, Emilly Gomes, Gabriela Mota, Daniele de Freitas, Camila Cristina e que acreditaram em pesquisar osteopatia mesmo sem saber muito o que fosse e o grupo de alunos do programa de extensão em reabilitação cardíaca: Gabriel Henrique, Vitória Soares Pinho, Bruna Urueña, Aliny Missias, Geovana Giardini, Gabriela Pagidis, Gabriela Souza que ajudou a tornar nosso programa e o laboratório uma realidade.

Aos professores que ajudaram e apoiaram este estudo: Maria Aparecida Catai pela prontidão e acolhimento na UFSCar, Gaspar Chiappa, Sérgio Mateus, Graziella Cipriano, Vera Paz, Kelb Bousquet, Guilherme Molina, Ricardo Jacó, Marisete Safons, Martim Bottaro, João Paulo Matheus, Rodrigo Carregaro, João Durigan.

Ao Colegiado do Curso de Fisioterapia da Universidade de Brasília por me proporcionar e me apoiar no afastamento para conclusão do doutorado. Agradeço a cada um pela ajuda.

Em especial e, por fim, ao meu orientador Prof. Gerson Cipriano Júnior. Primeiramente por acreditar nas minhas idéias, que, para quem não é osteopata, podem soar estranhas. Por passar o ideal de um trabalho em grupo de pesquisa, poder proporcionar ao longo do curso formação de pesquisa: como procurar, como interpretar, como tabular, planilhar, interpretar e escrever, deixando da forma mais simples possível. Por ajudar a proporcionar os recursos de pesquisa e nos estimular a essa busca. Ao longo desses 4 anos de curso eu falava que queria aprender começando do zero e tentar absorver o máximo possível, sem tentar queimar etapas, e ele com muito zelo, atenção, carinho e paciência se dedicou a cada passo. Espero poder sido 
um bom aluno da mesma maneira como ele foi um bom orientador. Aprendi muito como aluno, mas muito mais como pessoa. Fazer as coisas da melhor forma que você puder. Essa é a grande lição que o meu querido orientador e amigo me deixou. Minha eterna gratidão Professor.... 
"E no final, o amor que você receberá, Será igual àquele amor que você ofereceu" Lennon/McCartney 


\section{SUMÁRIO}

CAPÍTULOS

PÁGINA

1. LISTA DE TABELAS..................................................................... IX

2. LISTA DE FIGURAS …........................................................................ X

3. LISTA DE ABREVIAÇÕES, NOMENCLATURAS E SÍMBOLOS.............. XI

4. RESUMO ….................................................................................... XIII

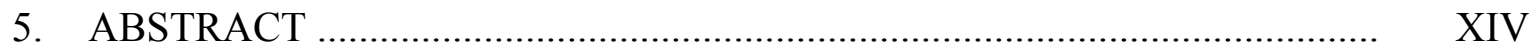

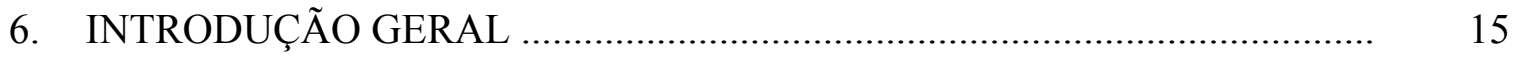

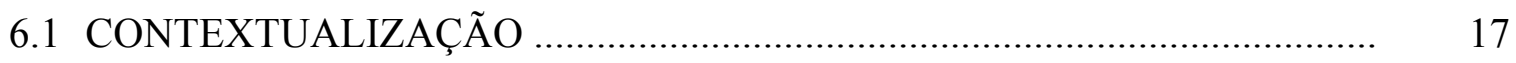

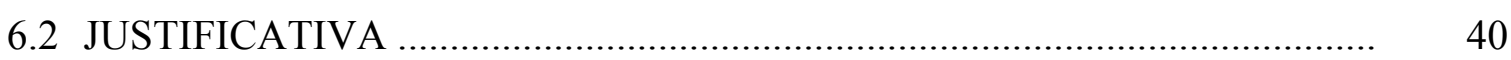

6.3 OBJETIVOS PROPOSTOS _................................................................ 40

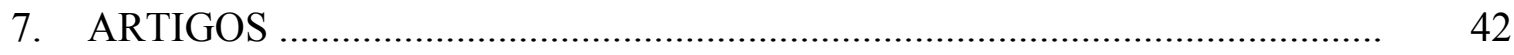

8. DISCUSSÃO GERAL E CONCLUSÕES ….................................................. 46

8.1 INTEGRAÇÃO DAS PARTES DO PROJETO ................................................. 46

8.2 DETALHES DA EXECUÇÃO DO PROJETO ……...................................... 49

8.3 CONCLUSÕES _............................................................................... 54

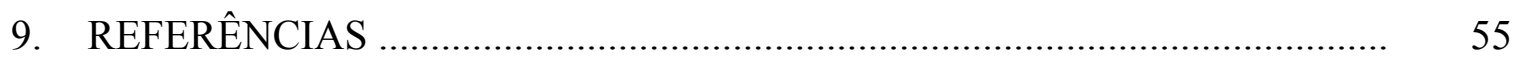

ANEXO 1 - PRODUÇÕES DURANTE O DOUTORADO ........................... 64

ANEXO 2 - PARECER DO COMITÊ DE ÉTICA E PESQUISA ................... 68

ANEXO 3 - REGISTRO BRASILEIRO DE ENSAIOS CLÍNICOS .............. $\quad 70$

ANEXO 4 - NORMAS DA REVISTA E QUALIS - MANUSCRITO 1 ........ 76

ANEXO 5 - NORMAS DA REVISTA E QUALIS - MANUSCRITO 2 ....... 83

ANEXO 6 - NORMAS DA REVISTA E QUALIS - MANUSCRITO 3 ........ 101

ANEXO 7 - COMPROVANTE DE SUBMISSÃO MANUSCRITO 3 .......... 115 
1. LISTA DE TABELAS

PÁGINA

1. VALORES DE NORMALIDADE DA VELOCIDADE VASCULAR ........... 28

2. VARIÁVEIS DESFECHO DA VFC E SUAS EXPLICAÇÕES .................... 39 
FIGURAS

PÁGINA

1. ESQUEMA DA FISIOLOGIA DA DILATAÇÃO MEDIADA PELO FLUXO 20

2. ELEMENTOS ESSENCIAIS PARA ANÁLISE DA DMF .............................. 21

3. ULTRASSOM PERPENDICULAR E NÃO PERPENDICULAR .................... 26

4. DIAGRAMA DA ANÁLISE ESPECTRAL DO ULTRASSOM COM DOPPLER

5. ESQUEMA REPRESENTATIVO DO SISTEMA NERVOSO AUTONÔMICO SIMPÁTICO E PARASSIMPÁTICO

6. TRAÇADO DO ELETROCARDIOGRAMA E O INTERVALO R-R.

7. COMPORTAMENTO DA VELOCIDADE MÉDIA DO VASO E ÍNDICE DE RESISTÊNCIA PRÉ, PÓS IMEDIATO E 10 MINUTOS DE PROSSEGUIMENTO.

8. TÉCNICA DE MANIPULAÇÃO CERVICAL EM ROTAÇÃO PARA A ESQUERDA E MANIPULAÇÃO DA PRIMEIRA COSTELA EM POSTERIORIDADE

9. TÉCNICA DE DESCOMPRESSÃO DA BASE DO CRÂNIO SEGUNDO MAGOUN

10. TÉCNICA DE ESTIRAMENTO DA PLEURA A ESQUERDA E TÉCNICA FUNCIONAL PARA O DIAFRAGMA

11. TÉCNICA DE DESCOMPRESSÃO CARDÍACA. PRIMEIRA FASE DE COMPRESSÃO E SEGUNDA FASE DE DESCOMPRESSÃO.

12. TÉCNICA DE BOMBEIO DO DUCTO TORÁCICO..

13. EQUIPAMENTO DE ULTRASSOM UTILIZADO NO ESTUDO

14. EXEMPLO DA COLETA DE DADOS EM VOLUNTÁRIO......

15. EXEMPLO DE ANÁLISE COM SOFTWARE DE DETECÇÃO DE BORDAS PARA A DMF

16. EXEMPLO DE ANÁLISE DE DOPPLER

17. EXEMPLO DE TACOGRAMA DE VARIABILIDADE DE FREQUÊNCIA CARDÍACA 


\section{LISTA DE ABREVIAÇÕES, NOMENCLATURAS E SÍMBOLOS}

\begin{tabular}{|c|c|}
\hline AR & Auto-Regressivo \\
\hline AUC & Área Abaixo da Curva \\
\hline AVBA & Alta velocidade e baixa amplitude \\
\hline $\mathrm{CP}$ & Condutância da Pele \\
\hline CTG & Grupo controle \\
\hline DMF & Dilatação mediada pelo fluxo \\
\hline DPGA & Área Cinzenta Dorsal Periaquedutal \\
\hline ECG & Eletrocardiograma \\
\hline EDV & Velocidade Diastólica Final \\
\hline eNO & Óxido nítrico sintetase endotelial \\
\hline eNOS & Óxido Nítrico Sintetase Endotelial \\
\hline $\mathrm{FC}$ & Frequência cardíaca \\
\hline FFT & Fast Fourrier Transform \\
\hline $\mathrm{HF}$ & Espectral Absoluta de Alta Frequência \\
\hline HMGB1 & High mobility group protein B1 \\
\hline HVLA & Alta Velocidade Baixa Amplitude \\
\hline $\mathrm{IC}$ & Insuficiência Cardíaca \\
\hline IP & Índice de Pulsabilidade \\
\hline IR & Índice de Resistência \\
\hline LF & Espectral absoluta de Baixa Frequência \\
\hline $\mathrm{LF} / \mathrm{HF}$ & Balanço autonômico \\
\hline MANOVA & Análise de variância múltipla \\
\hline MCA & Métodos Complementares Alternativos \\
\hline $\mathrm{MHz}$ & Mega Hertz \\
\hline MIA & Mobilização intra articular \\
\hline NHIS & National Health \\
\hline NO & Óxido Nítrico \\
\hline PA & Pressão Arterial \\
\hline $\mathrm{PCO}_{2}$ & Pressão de Gás Carbônico \\
\hline $\mathrm{pH}$ & Potencial de Hidrogênio \\
\hline $\mathrm{PO}_{2}$ & Pressão de Oxigênio \\
\hline PVS & Pico de Velocidade Sistólica \\
\hline RMSSD & Rooth Mean Square Standard Deviation \\
\hline SD1 & Desvio vertical \\
\hline SD2 & Desvio horizontal \\
\hline SDNN & Standard Deviation N-N \\
\hline SHG & Grupo sham \\
\hline SNA & Sistema Nervoso Autonômo \\
\hline SNP & Sistema Nervoso Parassimpático \\
\hline SNS & Sistema Nervoso Simpático \\
\hline SUS & Sistema Único de Saúde \\
\hline TART & Tensão, Assimetria, Restrição e Dor \\
\hline TENS & Transcutaneal Eletrical Neural Stimul \\
\hline
\end{tabular}


TMOG Grupo tratamento manipulativo osteopático

ULF Espectral Absoluta de Ultra Baixa Frequência

VDF Velocidade diastólica final

VFC Variabilidade da Frequência Cardíaca

VLF Espectral Absoluta de Frequência muito baixa 


\section{RESUMO}

Introdução: O Tratamento Manipulativo Osteopático (TMO) é dito capaz de modular o sistema vascular e o sistema nervoso autônomo (SNA). No entanto, do que é do nosso conhecimento, ainda não existe na literatura estudos associando as respostas hemodinâmicas e autonômicas pós TMO (lacuna 1). Contudo, ainda é controverso quais técnicas de TMO seriam melhores para modular o SNA (lacuna 2) e se é seguro receber o TMO sob o ponto de vista cardiovascular (lacuna 3).

Objetivo: Estudar as lacunas supracitadas.

Método: Lacuna 1: Ensaio clínico randomizado (ECR) comparando os efeitos do TMO na função vascular e autonômica - análise do dilatação fluxo mediada (DFM) e variabilidade da frequência cardíaca (VFC) - em cardiopatas. Lacuna 2: Revisão sistemática com metanálise (RSMA) acerca dos efeitos da tratamento manual da coluna vertebral (TMCV) no SNA (medidos por VFC e condutância da pele). Lacuna 3: ECR comparando a influência do TMO cervical em indivíduos saudáveis na pressão arterial (PA) e frequência cardíaca (FC) verificando a segurança de receber TMO.

Resultados: Lacuna 1: Verificou-se um aumento da DMF (pre $\mathrm{x}$ time course $14 \% \pm 2,29$ para $23,5 \% \pm 6,87 \mathrm{p}=0,027$ ), diferença absoluta (pre $\mathrm{x}$ time course $0,6 \mathrm{~mm}$ $\pm 0,159$ para $1,07 \pm 0,29 \mathrm{p}=0,03$ ) e diâmetro de pico (pre $\mathrm{x}$ time course $4,9 \mathrm{~mm} \pm 0,74$ para $5,67 \pm 0,76 \mathrm{p}=0,003$ and post $\mathrm{x}$ time course $5,2 \mathrm{~mm} \pm 0,58$ para $5,67 \pm 0,76$ $\mathrm{p}=0,02)$. Todavia, na função autonômica houve aumento dos índices vagais imediatamente após a técnica (RMSSD, SDNN e SD1) e após 10 minutos de followup a manutenção do aumento vagal além de aumento de índice simpático (LF). Lacuna 2: RSMA: A manipulação promove aumento da $L_{\text {norm }}$ e LF/HF. A mobilização intra articular aumenta a condutância da pele (durante a aplicação). Não há alteração do sistema parassimpático e FC após a manipulação. Supreendentemente, após a execução da mobilização intra articular, condutância da pele também não alterou. Lacuna 3: ECR: Sem alterações relevantes na PA e FC após TMO cervical em nenhum dos grupos imediatamente nem após 24 horas.

Conclusão: Lacuna 1: O TMO foi capaz de modular o sistema vascular e autonômico em pacientes com insuficiência cardíaca. Supreendentemente, os ajustes vasculares parecem ocorrer imediatamente após a aplicação da técnica, independente da modulação simpática. Lacuna 2: RSMA: As TMCV aumentam o SNA simpático, porém não altera o parassimpático nem a FC. Lacuna 3: ECR: O TMO cervical não produz alteração imediata e 24 horas significativas na PA e FC, mostrando ser seguro do ponto de vista cardiovascular.

Palavras-chaves: Tratamento manipulativo osteopático, variabilidade da frequência cardíaca, Sistema nervoso autônomo, insuficiência cardíaca e DMF. 


\section{ABSTRACT}

Introduction: Osteopathic Manipulative treatment (OMT) is said to be able to modulate the vascular system and the autonomic nervous system (ANS). However, what we know, does not yet exist in the literature studies associating the hemodynamic responses and post OMT autonomic (gap 1). However, it's still controversial which OMT techniques would be best to modulate the ANS (gap 2) and if it is safe to receive the OMT cardiovascular point of view (gap 3).

Objective: Study the above-mentioned gaps.

Method: Gap 1: randomized clinical trial (RCT) comparing the effects of OMT on vascular and Autonomic function-analysis of flow mediated dilation (FMD) and heart rate variability (HRV)-in cardiac patients. 2 gap: systematic review with metaanalysis (SRMA) about the effects of manual treatment of the spine (MTS) in ANS (measured by HRV and skin conductance). Gap 3: RCT comparing the influence of cervical OMT in healthy individuals in blood pressure (BP) and heart rate (HR) checking the safety of receiving OMT.

Results: Gap 1: FMD incresead (pre x time course $14 \% \pm 2.29$ to $23.5 \% \pm 6.87 \mathrm{p}=$ 0.027 ), absolute difference (pre $\mathrm{x}$ time course $0,6 \mathrm{~mm} \pm 1.07 \pm 0.29$ for $0.159 \mathrm{p}=$ 0.03 ) and diameter of peak (pre $\mathrm{x}$ time course $4,9 \mathrm{~mm} \pm 0.74$ to $5.67 \pm 0.76 \mathrm{p}=0.003$ and post $x$ time course $5 \mathrm{~mm} \pm 0.58$ to $5.67 \pm 0.76 \mathrm{p}=0.02$ ) also. However, vagal autonomic function indices increased immediately after the technique (RMSSD, SDNN and SD1) and after 10 minutes of follow-up increased vagal maintenance in addition to increased sympathetic (LF) index. 2 Gap: RSMA: Manipulation promotes increased $\mathrm{LF}_{\text {norm }}$ and $\mathrm{LF} / \mathrm{HF}$. Intra articular mobilization improves skin conductance (during the application). No change of the parasympathetic system and HR after the manipulation. Amazingly, after the execution of the intra articular mobilization, skin conductance also did not change. Gap 3: RCT: Without relevant changes in BP and HR after cervical OMT in any of the groups immediately or after 24 hours.

Conclusion: Gap 1: the OMT was able to modulate the vascular and Autonomic system in patients with heart failure. Amazingly, the vascular adjustments seem to occur immediately after the application of the technique, independent of the sympathetic modulation. 2 gap: SRMA: MTS increase sympathetic ANS, but does not change the parasympathetic or HR. Gap 3: RCT: The cervical OMT does not produce immediate change and 24 hours in BP and HR significant showing be safe on cardiovascular point of view.

Keywords: Osteopathic manipulative Treatment, heart rate variability, autonomic nervous System, heart failure and DMF. 


\section{INTRODUÇÃO}

A Osteopatia reconhece a importância do sistema nervoso autônomo (SNA) na função corporal e suas relações com a saúde e a doença. O tratamento manipulativo osteopático foi desenvolvido em 1874 pelo médico americano Andrew Taylor Still, o qual se tornou uma medicina alternativa àquela convencional. Enquanto os médicos alopatas buscam novas medicações para tratar patógenos específicos, os osteopatas desenvolvem técnicas e estratégias manuais para tratar as causas funcionais das patologias. Várias pessoas são submetidas, todos os dias, ao tratamento manipulativo osteopático por todo o mundo. Pessoas fisicamente ativas, idosos e cardiopatas são alguns exemplos (1-4).

Existem inúmeras opções de técnicas de tratamento manipulativo osteopático. As técnicas de alta velocidade e baixa amplitude (AVBA) - manipulação da coluna, técnicas de mobilização intra articular (MIA), técnicas miofasciais, além das técnicas viscerais e cranianas estão dentro do escopo da osteopatia. Dentre elas, as técnicas miofasciais realizadas na próximas a base do crânio e na coluna vertebral parecem produzir efeitos no grau de modulação autonômica. Giles e cols demonstraram aumento do grau de modulação vagal em 23 indivíduos jovens e saudáveis na posição supina. Da mesma forma, Henley e cols também verificaram o aumento do grau de modulação vagal após a técnica para liberação miofascial cervical no tilt test $(5,6)$. Ruffini e cols verificaram o mesmo resultado parassimpático após o tratamento manipulativo osteopático (7). Entretanto, as técnicas de AVBA cervical e torácica em indivíduos saudáveis parece levar a um aumento do balanço autonômico - com aumento da atividade simpática $(8,9)$. Em contraste, as técnicas de AVBA lombares em indivíduos sem dor pode levar a um aumento da atividade parassimpática $(10,11)$. As técnicas de MIA promovem aumento da atividade simpática durante a técnica, além de aumentar a frequência cardíaca (FC) e pressão arterial (PA), contudo a manutenção desses estímulos após o tratamento ainda é questionável (12).

A capacidade de provocar modulação autonômica após o tratamento manipulativo osteopático pode provocar secundariamente alterações vasculares. $\mathrm{O}$ uso de técnica AVBA na coluna lombar em sujeitos saudáveis proporcionou um aumento do fluxo sanguíneo cutâneo no dermátomo correspondente ao local da manipulação. Tal modulação vascular foi atribuída as mudanças autonômicas geradas pela técnica 
AVBA (13). Em sujeitos com doença arterial periférica, tratados por 2 meses com sessões de tratamento manipulativo osteopático a cada 15 dias com técnicas de liberação miofascial, técnicas de músculo energia, técnicas de AVBA (geralmente em T10 a L1), técnicas de bombeio linfático e técnicas craniana, foi verificado que ao final do tratamento houve melhora em medidas inflamatórias vasculares, na resposta da dilatação mediada pelo fluxo (DMF) da artéria braquial mantida por 6 meses (14). Entretanto, ainda existe dúvida acerca da segurança da técnica de AVBA e MIA nas respostas cardiovasculares (FC e PA) (12). Em indivíduos com patologias vasculares e cardíacas o benefício do tratamento manipulativo osteopático ainda é questionável.

Dentre os estudos de osteopatia na área da cardiologia, em pessoas hipertensas tratadas com tratamento manipulativo osteopático e acompanhadas por 1 ano houve diminuição da PA sistólica e da camada íntima média arterial da carótida. Tais mudanças podem ser explicadas por modulação autonômica e/ou diminuição das citocinas inflamatórias locais (15). Contudo, a maioria dos estudos ainda apresentam baixa qualidade metodológica. Os trabalhos com menor risco de viés demonstraram pequenas mudanças da PA depois do tratamento manipulativo osteopático (16). Em pacientes com problemas cardíacos mais graves como a insuficiência cardíaca (IC), o tratamento manipulativo osteopático poderia ser benéfico, porém a literatura é escassa nesse assunto.

Os benefícios que o tratamento manipulativo osteopático pode promover aos pacientes com IC podem surgir por conta da gênese dessa patologia. As anormalidades do sistema hemodinâmico central e as alterações na função muscular esquelética são as principais, porém alterações reflexas no SNA também desempenham um papel importante não só na origem dos sintomas limitantes do exercício como também na progressão da IC. Tais pacientes apresentam a atividade simpática aumentada e parecem ter também a diminuição da atividade parassimpática vagal (17-19). O sistema parassimpático tem efeito anti-inflamatório mediado pela ativação do alfa7 receptor nicotínico da acetilcolina. Essa ativação inibe a liberação de citocinas e da HMGB1, mediador da inflamação dos macrófagos que, de forma persistente, pode levar a progressiva disfunção ventricular esquerda, contribuindo na sua remodelação e na apoptose de suas células (19-21). 
Diversas formas de tratamento têm sido utilizadas na tentativa de regular a atividade autônoma nos pacientes com IC. Sabe-se que o aumento do grau de modulação do SNA simpático é capaz de produzir diversos efeitos fisiológicos, tais como: ajuste da frequência cardíaca, regulação do sistema renina-angiotensinaaldosterona, melhora da sensibilidade baroreflexa, supressão de citocinas pró inflamatórias, normalização da via de sinalização do óxido nítrico e supressão do gap de remodelação ventricular. Esses efeitos têm sido relacionados a alguns benefícios clínicos nos pacientes de IC, tais como: melhora da capacidade funcional, melhora da qualidade de vida e morbi mortalidade (19).

Encontra-se na literatura distintas formas de tratamento para a IC, entre elas modalidades clínicas e as modalidades complementares e alternativas (MCA). Destaca-se desde os anos 1970 o uso da terapia betabloqueadora com o propanolol e o aprenolol na modalidade clínica. Nos anos 1990 surgiram os estudos randomizados prospectivos demostrando os benefícios dessa terapia na morbi mortalidade para esses pacientes (22). Entretanto, o National Health Interview Survey listou as mais frequentes terapias de MCA para tratamento da IC: uso de óleo de peixe / ômega 3, glucosamina, equinácea e linhaça $(17,7 \%)$, respiração profunda $(12,7 \%)$, meditação $(9,4 \%)$, quiropraxia e osteopatia (8,6\%), e massagem (8,3\%). O uso das MCA entre os adultos é maior entre as mulheres e os de meia-idade que são mais instruídas e têm rendimentos mais elevados. A osteopatia ainda tem sido usada para tratar as dores musculoesqueléticas associadas a condição da doença cardiovascular (23).

\subsection{CONTEXTUALIZAÇÃO}

A possibilidade de modulações vasculares que são atribuídas ao tratamento manipulativo osteopático e outros tratamentos manipulativos da coluna vertebral se baseiam em duas teorias: 1 - por meio de possíveis mudanças autonômicas, que por mecanismo reflexo do sistema autônomo simpático no vaso levará a alteração do diâmetro da artéria promovendo mudanças na DMF e características do fluxo e 2 por conta das alterações intrínsecas no sistema vascular pós tratamento manipulativo osteopático, pela diminuição dos mediadores inflamatórios do vaso e aumento da oferta de óxido nítrico (NO) vascular. As alterações do grau de modulação autonômica se baseiam na proximidade anatômica dos gânglios vertebrais 
autonômicos (cadeia latero vertebral simpática) com a coluna vertebral e/ou por conta da neuromodulação eferente oriundo do tronco cerebral que é estimulado pelas aferências somáticas das manipulações.

Nesta revisão serão apresentados os mecanismos de DMF e doppler assim como a anatomia e fisiologia do sistema nervoso autônomo. Incluindo a relação do SNA com o sistema cardiovascular e com a insuficiência cardíaca. Além disso, uma das suas principais formas de mensuração e a Variabilidade da Frequência Cardíaca.

\subsubsection{OSTEOPATIA NO TRATAMENTO CARDIOVASCULAR}

A osteopatia é um sistema de avaliação e tratamento com metodologia e filosofia própria baseado na interdependência da estrutura e função do organismo. Foi desenvolvida pelo médico americano Andrew Taylor Still em 1874. Desde então, a osteopatia baseia-se em um forte racional anatômico para justificar e tratar os sintomas e patologias. A abordagem do paciente para o osteopata é global, em uma visão holística de funcionamento corporal. Várias possibilidades de tratamento são atribuídos a osteopatia desde dores músculo esqueléticas até doenças viscerais, entre elas problemas ginecológicos, intestinais, estomacais, pulmonares e cardiovasculares e do sistema nervoso autônomo. $(4,24)$

A intervenção do osteopata para as doenças cardíacas e do sistema nervoso autonômico baseiam-se na estrutura e função cardiovascular e autonômica. As disfunções somáticas do sistema músculo esquelético podem gerar alteração do tônus autonômico de base e influenciar o funcionamento visceral de forma geral. Para o sistema cardiovascular, tais disfunções na região da coluna cervical e torácica, responsáveis pela inervação simpática do coração, e na base do crânio (passagem da inervação parassimpática cardíaca) podem influenciar de forma negativa o funcionamento cardiovascular. Além disso, todo o sistema arterial está em íntima relação com o sistema nervoso autônomo simpático, visto que este inerva e é uma das formas de controle do vaso. $(2,24)$

Para se influenciar esse mecanismo fisiológico descrito, o osteopata utiliza de técnicas que possa melhorar essa disfunção somática melhorando essa alteração de tônus autonômico. As manipulações articulares da coluna de alta velocidade e baixa amplitude (AVBA), as técnicas de mobilização intra articular (MIA), técnicas 
cranianas, miofasciais, técnicas fasciais estruturais e funcionais e viscerais são algumas das possibilidades terapêuticas. As técnicas para tratamento da função cardiovascular incluem técnicas na coluna cervical e torácica, base do crânio, diafragma, ducto torácico, relação com as pleuras pulmonares e das fáscias cardíacas. De forma geral, melhorar a mecânica envolvida no movimento do diafragma, do deslizamento do coração entre as fáscias pulmonares, da relação com o esterno e seus ligamentos com o coração e da inervação autonômica são as bases do tratamento osteopático para a patologia cardíaca de forma geral. (25)

\subsubsection{DILATAÇÃO MEDIADA PELO FLUXO - DMF}

\subsubsection{PERSPECTIVA HISTÓRICA}

Celermjer e colaboradores (1992) desenvolveram a técnica de DMF. Um método não invasivo sendo dito capaz de medir a função do endotélio vascular realizada por meio do uso de ultrassom em resposta a hiperemia induzida por oclusão. Apresenta-se como medição fiável, e se correlaciona com a função endotelial invasiva avaliada nas artérias coronárias. Num esforço de padronização Corretti e cols em 2002 publicaram as primeiras orientações da metodologia de DMF para a artéria braquial, que até agora têm sido referenciadas mais de 1.000 vezes. Pyke e Tschakovsky sugeriram atualizar as diretrizes apresentadas por Corretti e cols, desde então essas seriam as principais referências acerca das recomendações de uso $(26,27)$.

\subsubsection{FISIOLOGIA DA DMF}

Durante um teste de DMF, a vasodilatação ocorre em seguida de um aumento agudo no fluxo sanguíneo tipicamente induzida pela paragem circulatória do braço, com um manguito com oclusão supra-sistólica, durante um período de tempo. Tal situação aumenta a hiperemia laminar com forças de corte paralelos ao eixo longitudinal do vaso, que é traduzido via mecanorreceptores luminal da célula endotelial. Em seguida há um aumento da expressão de proteína $G$, de fosfoquinase A, um aumento de sinalização de óxido nítrico sintetase endotelial (eNOS), o que promove atividade catalisadora na conversão da L-arginina em NO. O NO difunde-se para a camada média ativando a guanilato ciclase solúvel que converte guanosina trifosfato em guanosina monofosfato para induzir o relaxamento do músculo liso por conta da diminuição da concentração de cálcio e vasodilatação subsequente. Na sua 
forma mais tradicional, o aumento do diâmetro arterial, como consequência da hiperemia reativa, é comparado com o diâmetro basal e expresso simplesmente como percentagem deste diâmetro basal (\% DMF) $(27,28)$. A Figura 1 mostra o esquema dessa fisiologia.

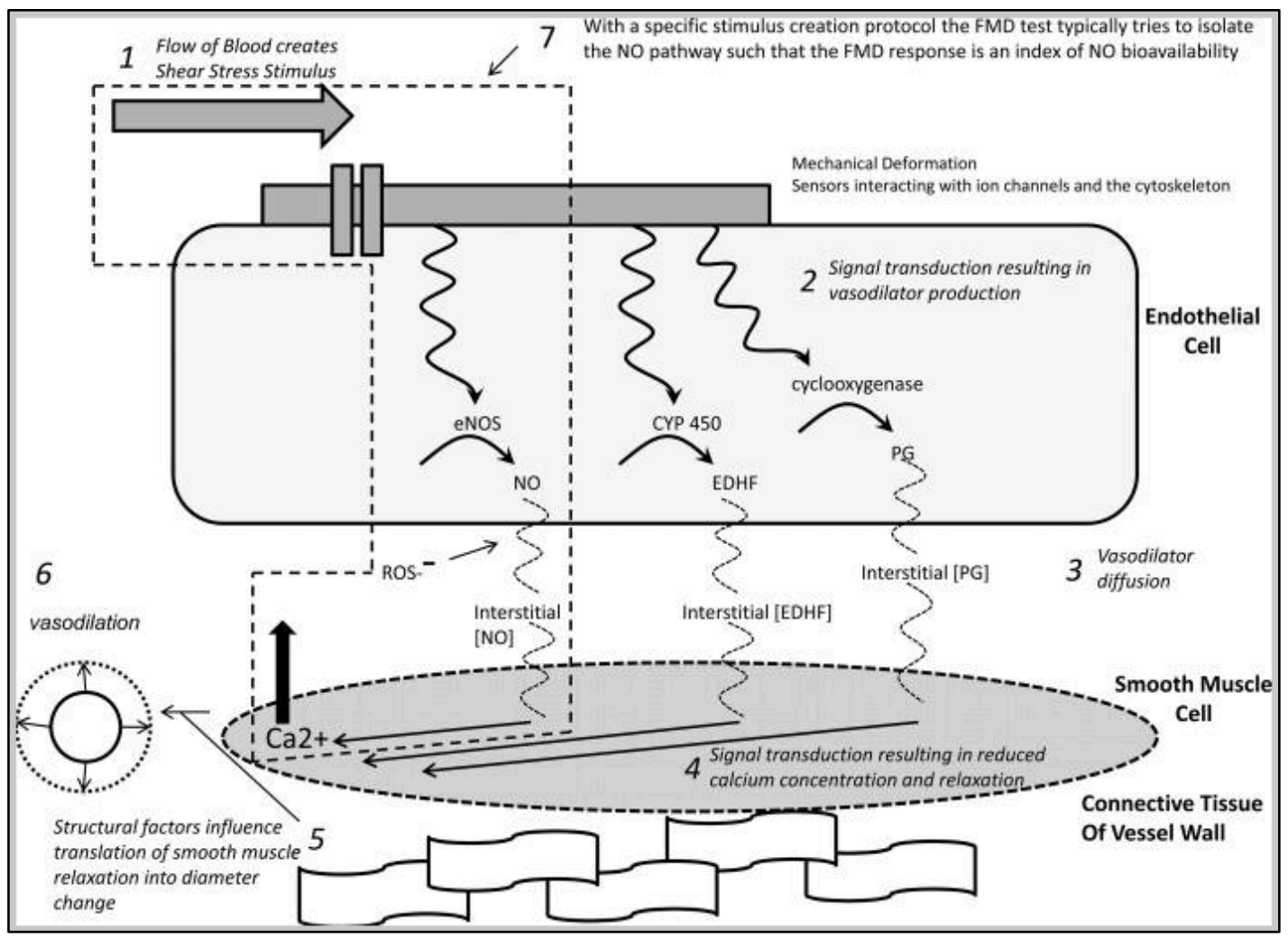

Figura 1. Esquema da fisiologia da dilatação mediada pelo fluxo.

Legenda: 1 - fluxo de sangue cria um estímulo de estresse de cisalhamento no vaso. 2 - A transdução do sinal resultando em produção de vasodilatação. 3 - Difusão vasodilatadora. 4 - O sinal de transdução resultando em redução da concentração de cálcio e relaxamento. 5 - Fatores estruturais influenciam o relaxamento muscular para a mudança do diâmetro do vaso.

Fonte: Modificado de Thijssen et al. 2011.

\subsubsection{ELEMENTOS ESSENCIAIS NA MEDIDA DA DMF}

A figura 2 demonstra os elementos primordiais para a medida da DMF de maneira esquemática. 


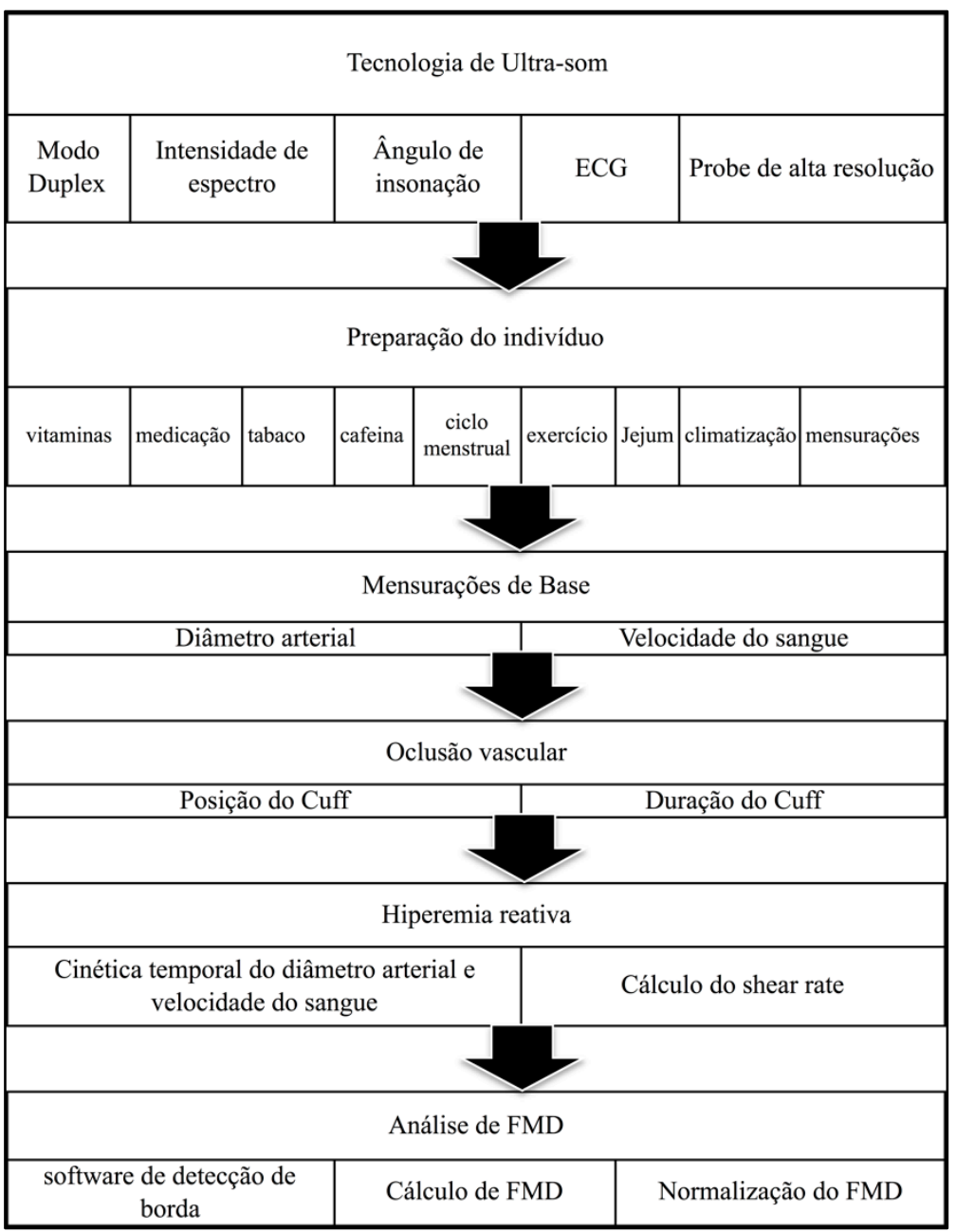

Figura 2. Elementos essenciais para análise da DMF.

Fonte: adaptado de Harris et al., 2010.

\subsubsection{ULTRASSOM}

A medição precisa da DMF é altamente dependente da identificação da parede arterial definida, o que requer imagem de ultrassom de alta resolução. Cada sonda de ultrassom é classificada de acordo com a gama de frequências em megahertz $(\mathrm{MHz})$, que é inversamente proporcional à profundidade da captação da imagem. Para os vasos mais superficiais seria ideal transdutores lineares variantes de 10-14 MHz, atualmente considerada "alta resolução". O modo duplex permite a aquisição simultânea do diâmetro do vaso e velocidade do sangue, respectivamente. Isto permite o cálculo da velocidade de corte, para qualquer período de tempo determinado a partir dos quais a integral de cisalhamento ao longo do tempo, isto é, a área de taxa de cisalhamento sob a curva (AUC - area under the curve) pode ser calculada (29). 
O ângulo de insonação para o vaso sanguíneo que corre paralelo à superfície cutânea (por exemplo, da artéria braquial) é de 90 graus, porém este é o pior cenário para a avaliação doppler da velocidade do sangue. Alguns comprometimentos da qualidade da imagem, balanço do transdutor para cima em uma extremidade mais do que o outro (conhecido como ajustes de calcanhar/dedos) fará com que o vaso pareça mover na diagonal do monitor a um ângulo inferior a 90 graus. Por vezes essa angulação não fica disponível na coleta, então verificou-se que, embora seja recomendado o ângulo de insonação de $60^{\circ}$, o maior ângulo normalmente aceito na literatura é de $70^{\circ}(30,31)$.

Dependendo da rigidez do vaso e da pressão da pulsação o diâmetro arterial pode variar dentro de um único ciclo cardíaco e isso pode confundir completamente a avaliação da DMF. Os sistemas de ultrassom Doppler que possuem um eletrocardiograma (ECG) integrado facilita a avaliação do diâmetro de acordo com o ciclo cardíaco (por exemplo diástole final). Caso não esteja disponível no próprio sistema de ultrassom, um ECG externo pode ser utilizado (32).

Existem inúmeros métodos de cálculos para a velocidade do sangue. A maneira mais simples utiliza o envelope exterior do espectro Doppler para determinar a velocidade média de pico, enquanto que a abordagem um pouco mais complexa integra a área sob este envelope para calcular a velocidade média de pico. No entanto, nenhum desses cálculos reflete com precisão a gama complexa e variável de velocidades e as suas distribuições relativas dentro dos espectros de Doppler. Portanto, para avaliar com precisão a velocidade do sangue, recomenda-se que a intensidade ponderada dos cálculos do tempo médio sejam realizadas para refletir com maior acurácia a contribuição de células vermelhas que se deslocam a velocidades diferentes no interior do vaso (33).

\subsubsection{PREPARAÇÃO DO SUJEITO}

O exercício prévio ou o estado descansado pode influenciar nos resultados, portanto recomenda-se que os voluntários não realizem atividade física por pelo menos 12 horas antes da medição de DMF. Uma única sessão de exercício foi documentada para melhorar a DMF em adultos saudáveis, nos homens com excesso de peso e nas mulheres pós-menopausa (34-36). Além disso, as avaliações da DMF devem ser realizadas sob condições de jejum; no entanto, se o jejum não é possível, 
uma refeição padronizada baixo teor de gordura pode ser consumida antes da medição da DMF. O consumo de uma única refeição rica em gorduras e carboidratos é capaz de atenuar a DMF em indivíduos aparentemente saudáveis e diabéticos do tipo II $(37,38)$.

A climatização adequada também se faz importante. Recomenda-se que os indivíduos permanecem na posição em que o estudo será efetuado durante pelo menos 10 minutos em ambiente controlado silencioso com temperatura de 22 a $24^{\circ}$ celsius). Além disso, uma visita de familiarização separada dos procedimentos é recomendada para limitar a atividade simpática induzida pelo stress no dia de medição real (39). Dentre as medidas repetidas, vários testes de DMF pode ser realizados com intervalo de 30 minutos entre cada medida. As medidas de DMF apresentam variação diurna, portanto, devem ser realizadas na mesma hora do dia (40).

A suplementação vitamínica pode ser outro fator complicador. Os indivíduos devem se abster de suplementação de vitamina para até 72 horas antes da DMF. Existe evidência direta da redução na circulação de radicais livres após a suplementação antioxidante oral (vitamina C, vitamina E entre outras) (37,38). Assim como as medicações que recomenda-se ser descontinuada de 1 e 3 dias antes de uma medição da DMF, porém essa cessão da medicação pode não ser viável, portanto devendo ser reconhecida e documentada (41).

Para aqueles indivíduos que fumam, recomenda-se que não fumem nem se exponham a fumaça do cigarro por pelo menos 12 horas antes das medições de DMF (41). A mesma recomendação vale para a cafeína, pois ela inibe a guanilato ciclase solúvel $(42,43)$. Para as voluntárias do gênero feminino, o ciclo menstrual deve estar no mesmo período para a realização da DMF. A produção endógena de estrógeno e progesterona aumenta a atividade da eNOS e capacidade antioxidante influenciando a resposta vasodilatadora (44).

\subsubsection{AS MEDIDAS NA OCLUSÃO E NA LIBERAÇÃO DO}

\section{MANGUITO}

Para a mensuração das medidas basais e prévias da DMF, a avaliação precisa de um diâmetro médio e das velocidades de sangue simultâneas, com volume de amostra adequada, por pelo menos 10 ciclos cardíacos, e é recomendada antes de 
oclusão vascular. Nas medições da oclusão vascular recomenda-se que tanto os dados de diâmetro e velocidade devem ser adquiridos por pelo menos 10 segundos antes de soltar a braçadeira e continuar por pelo menos 2 minutos. Isso permitirá a documentação do diâmetro de pico, análise quantitativa das AUC de cisalhamento. Relatar o momento de pico de vasodilatação pode avaliar a função endotelial mais adequadamente ao se comparar diferentes grupos $(39,45)$.

O software de detecção de borda foi validado, de maneira independente entre dois avaliadores, e é recomendado para a medição do diâmetro arterial. Para fins de padronização, a obtenção de diâmetros deve ser feita por meio de gravação digital de dados contínua e análise não sequencial utilizando software de detecção de bordas. $\mathrm{O}$ cálculo de resposta a DMF, com o diâmetro "verdadeiro" de pico, é obtido e expresso como um aumento na vasodilatação acima de valores de referência. A normalização da DMF pelo cisalhamento (AUC) ainda é incerta. Além disso, o tempo para se obter o pico de vasodilatação pode ser um indicador importante (46). Se o software de detecção de borda não está disponível, recomenda-se que os dados (diâmetro e velocidade) sejam coletados a cada 4 segundos para os primeiros 20 segundos após liberação do manguito, seguido por cada 10 segundos para o período de coleta de dados dois minutos restantes $(47,48)$.

O cálculo da DMF como variação percentual utiliza a seguinte fórmula:

$$
\operatorname{DMF}(\%)=(\text { Diâmetro de pico }- \text { Diâmetro de base }) \times 100
$$

Diâmetro de base 


\subsubsection{DOPPLER}

\subsubsection{INTRODUÇÃO A ULTRASSONOGRAFIA VASCULAR}

O fluxo de sangue através de um vaso é aferido pela diferença de pressão entre as extremidades do vaso e pela resistência apresentada pela parede do vaso. Cada vaso normal no corpo humano tem padrão de fluxo característico representável em formas de onda espectrais obtidos com a ultrassonografia doppler (49). Os sinais de ultrassom refletidos em superfícies fixas tendem a manter a mesma frequência com que foram transmitidos, porém a ultrassonografia com doppler reflete sinais de objetos em movimento tais como os glóbulos vermelhos e suas mudanças de velocidade. A saída de uma ultrassonografia Doppler é por uma onda contínua e, normalmente, apresenta um sinal sonoro sempre que se tenha circulação na artéria examinada $(39,45)$.

O scanner duplex faz análise espectral, delimita a gama de frequências, que são as velocidades do fluxo sanguíneo encontradas na forma de onda arterial durante um ciclo cardíaco. A forma de onda normal de velocidade doppler é composta de três componentes que correspondem a diferentes fases do fluxo arterial: fluxo anterógrado rápido, atingindo um pico durante a sístole, uma reversão transitória do fluxo durante a diástole precoce, e fluxo anterógrado mais lento durante o final de diástole (29).

O feixe de som dirigido perpendicularmente à superfície alvo de interesse para a obtenção da eco imagem, aparece mais brilhante com escalas cinzentas. Com angulação perpendicular, as imagens são mais facilmente obtidas porque as artérias são, geralmente, paralelas ao transdutor. Para o componente doppler duplex, um ângulo de 60 graus entre o feixe de insonação e a parede do vaso deve ser mantida. Isto torna o ângulo de Doppler de importante consideração, pois os dados de velocidade podem também ser utilizados para classificar doenças e ângulos acima de 60 graus pode resultar em medidas superestimadas da velocidade e, por isso, devem ser evitados conforme mostrado na figura 3 (50). 


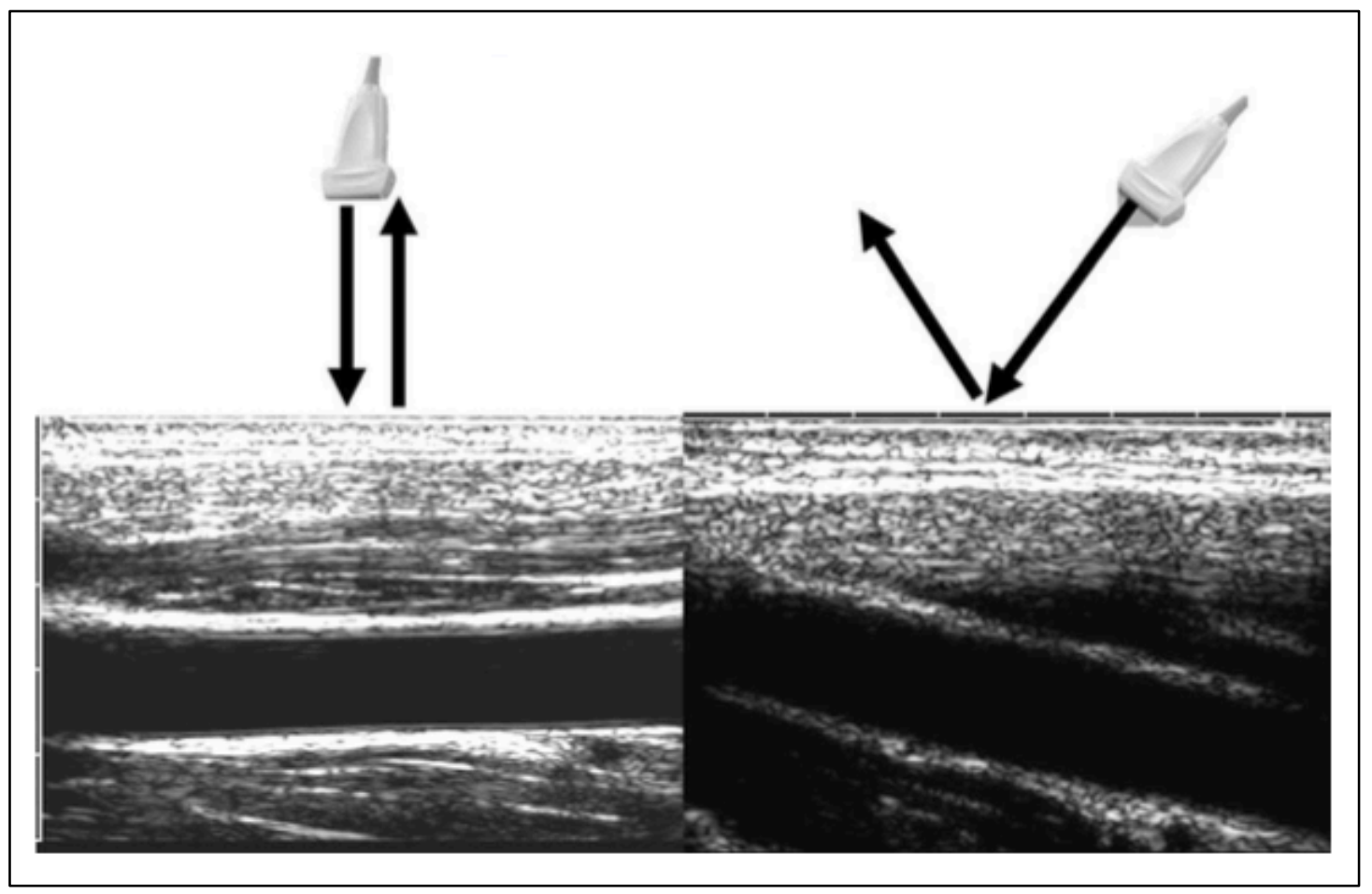

Figura 3. Ultrassom perpendicular e não perpendicular.

Fonte: modificado de Gerhard-Herman et al. 2006

\subsubsection{CÁLCULO DOS ÍNDICES DE FLUXO ARTERIAL}

O índice de resistência (IR) pode ser calculada a partir de medições espectrais usando a equação IR = (PVS - VDF) / PVS, sendo que PVS é o pico de velocidade sistólica e VDF é a velocidade diastólica final. O índice de pulsatilidade (IP) pode ser calculada usando a equação IP $=(P V S-V D F) / V M$, onde VM é a velocidade média de fluxo durante o ciclo cardíaco (33). Figura 4 ilustra a onda espectral.

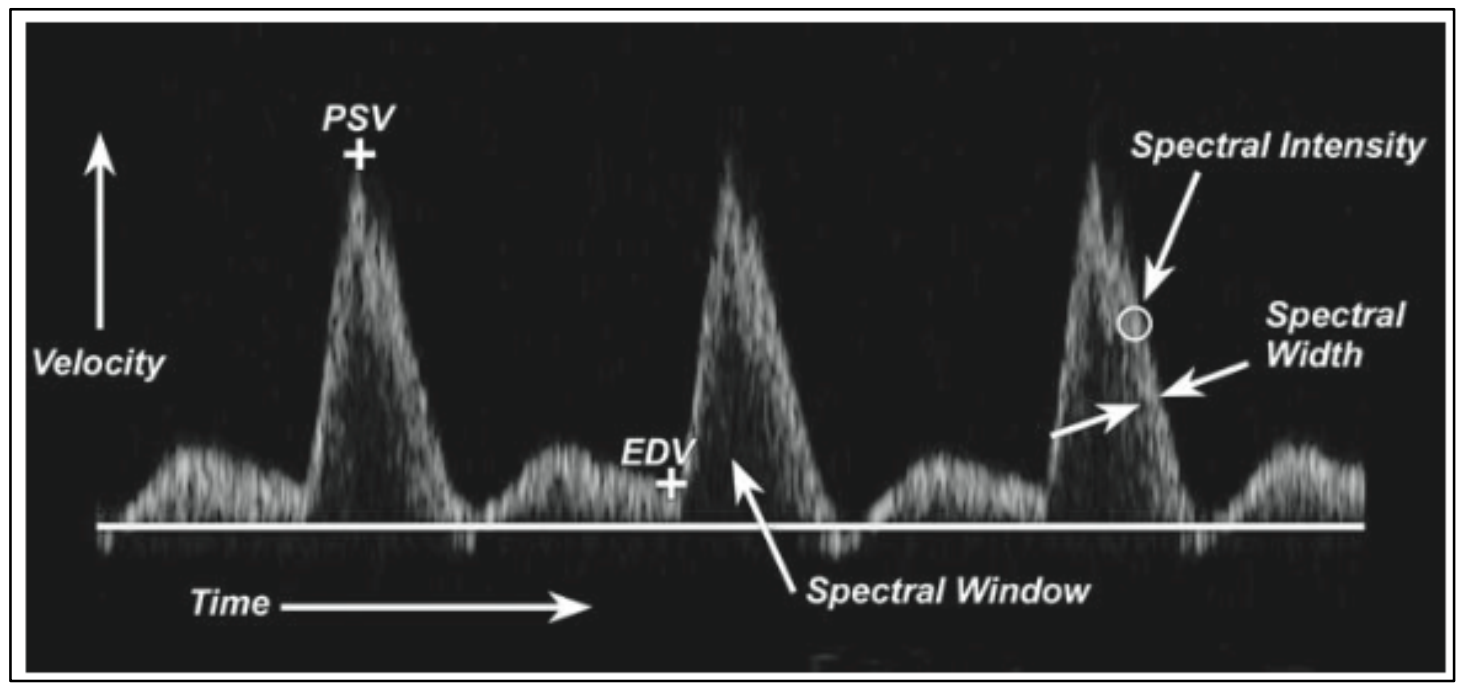


Figura 4. Diagrama da análise espectral do ultrassom com doppler. PVS - pico de velocidade sistólica, VDF - velocidade diastólica final. Spectral intensity Intensidade escpectral, Spectral window - janela espectral, Spectral width, largura espectral, velocity - velocidade e time - tempo.

Fonte: modificado de Chavhan et al., 2008.

O IR e o IP fornecem informações sobre o fluxo de sangue e resistência vascular que não pode ser obtida a partir de medições de velocidade absoluta. Os efeitos da variação e angulação do vaso são anulados nos cálculos desses índices. Várias formas de ondas anormais podem ser comparados por meio do cálculo do IR e do IP. Eventos fisiológicos que alteram a resistência vascular incluem: exercício físico, mudanças de orientação gravidade e nível de estresse, digestão entre outros (51).

\subsubsection{PADRÕES DE FLUXO DOPPLER NORMAIS}

Em fluxo de tomada, todas as células vermelhas do sangue se movem com a mesma velocidade, produzindo uma frente de onda plana. O espectro de Doppler a partir deste padrão de fluxo é caracterizado pela linha espectral estreita e uma janela espectral transparente, o qual representa a ausência de velocidades mais baixas. Esta forma de onda é tipicamente vista em grandes artérias, tais como a aorta $(26,51,52)$.

As células vermelhas do sangue periférico movem com velocidade mais lenta do que os glóbulos vermelhos centrais devido o atrito oferecido pela parede do vaso. Essa diferença de velocidades produz a frente de onda parabólica. No espectros de doppler, ocorrerá o alargamento da linha espectral e enchimento da janela espectral de forma de onda. Este padrão geralmente é visto em vasos com um diâmetro inferior a 5 $\mathrm{mm}(26,51,52)$.

O fluxo turbulento consiste em ampla gama de velocidades. Inclui componentes de fluxo invertido e é facilmente apreciado como várias cores nas imagens por doppler colorido. É visível como alargamento espectral com componentes abaixo do fluxo basal. A turbulência perto das bifurcações é considerada normal, porém longe delas é sugestivo de alguma anormalidade $(26,51,52)$. A tabela 1 contém os valores de normalidade da velocidade vascular arterial em algumas artérias da cabeça e pescoço, membros superiores e membros inferiores. 


\begin{tabular}{cccc}
\hline Artéria & $\begin{array}{c}\text { PVS } \\
(\mathrm{cm} / \mathrm{s})\end{array}$ & $\begin{array}{c}\text { VDF } \\
(\mathrm{cm} / \mathrm{s})\end{array}$ & IR \\
\hline Carótida externa & 57 a 87 & 11 a 21 & 0,72 a 0,84 \\
Carótida interna & 62 a 90 & 23 a 37 & 0,54 a 0,66 \\
Carótida comum & 78 a 118 & 20 a 32 & 0,72 a 0,84 \\
Femoral comum & 100 & - & 1,37 \\
Femoral superficial & 80 a 90 & - & 1,38 \\
Poplítea & 70 & - & 1,41 \\
& & & \\
Subclávia & 105 & - & - \\
Axilar & 80 & - & - \\
Braquial & 60 & - & - \\
\hline
\end{tabular}

Tabela 1. Valores de normalidade da velocidade vascular.

Legendas: PVS - pico de velocidade sistólica, VDF - velocidade diastólica final, IR índice de resistência. - Dados não fornecidos pela literatura.

Fonte: modificado de Gerhard-Herman et. al, 2006 (50) e Holland et. al, 1998.(53)

\subsubsection{SISTEMA NERVOSO AUTÔNOMO}

O SNA é composto pelos nervos e centros nervosos que comandam a vida vegetativa. O termo sistema nervoso autonômico foi descrito por John Newport Langley, em 1898, que considerava este sistema como independente das regiões não vegetativas do sistema nervoso central (54). Ainda é descrito classicamente como sistema motor visceral, inervando a musculatura lisa, o músculo cardíaco modificado (o tecido cardíaco intrínseco encarregado de estimulação e condução dos impulsos nervosos) e das glândulas. As fibras eferentes viscerais se acompanham das aferentes viscerais. Os ramos aferentes são condutores dos impulsos nervosos do sistema doloroso visceral, portanto estas fibras tem papel fundamental na regulação da função visceral. $(55,56)$.

Os ramos eferentes e os gânglios do SNA se organizam dentro de dois sistemas ou divisões denominados sistema nervoso simpático (SNS) e sistema nervoso parassimpático (SNP). A condução dos impulsos nervosos desde o SNC até o órgão efetor dependerá de uma série de neurônios dentro desse sistema. Existem dois neurônios para transmitir a informação pelo SNA. O corpo celular do primeiro neurônio (pré-sináptica ou pré-ganglionar) se encontra dentro da substância cinzenta 
na medula espinhal. Seu axônio somente estabelecerá sinapses com corpos celulares de neurônios (pós-sinápticos ou pós-ganglionares), que seriam os segundos neurônios do sistema. O segundo neurônio sempre estará fora do SNC, sendo em um gânglio laterovertebral, no caso do SNS ou no órgão efetor, incluindo músculo liso, cardíaco especializado e as glândulas, que fazem parte do SNP $(57,58)$.

Essa diferenciação leva a um entendimento sobre a diferença entre as fibras pré-ganglionares e pós-ganglionares. No SNS as fibras pré-ganglionares são curtas, enquanto que as fibras pós-ganglionares são longas para poder atingir o órgão alvo do nervo. Já no SNP as fibras pré-ganglionares são fibras longas enquanto que as pósganglionares são fibras curtas, pois estão próximas ou no órgão alvo do sistema. Além disso, a divisão simpática e a parassimpática tem uma diferença em relação aos neurotransmissores. O SNS utiliza a noradrenalina como neurotransmissor (exceto as glândulas sudoríparas) e o SNP, a acetilcolina $(56,58)$.

O SNS e o SNP têm funções antagônicas em relação ao funcionamento visceral torácico, abdominal e craniano. De forma geral, o SNS atua aumentando o funcionamento das vísceras supra diafragmáticas (torácicas) e diminuindo as vísceras infradiafragmáticas (abdominal), sendo que o SNP atua de forma oposta. Em relação ao crânio, o SNP estimula o funcionamento das glândulas e o SNS diminui o seu funcionamento (59). A figura 8 mostra a divisão do SNS e SNP. 


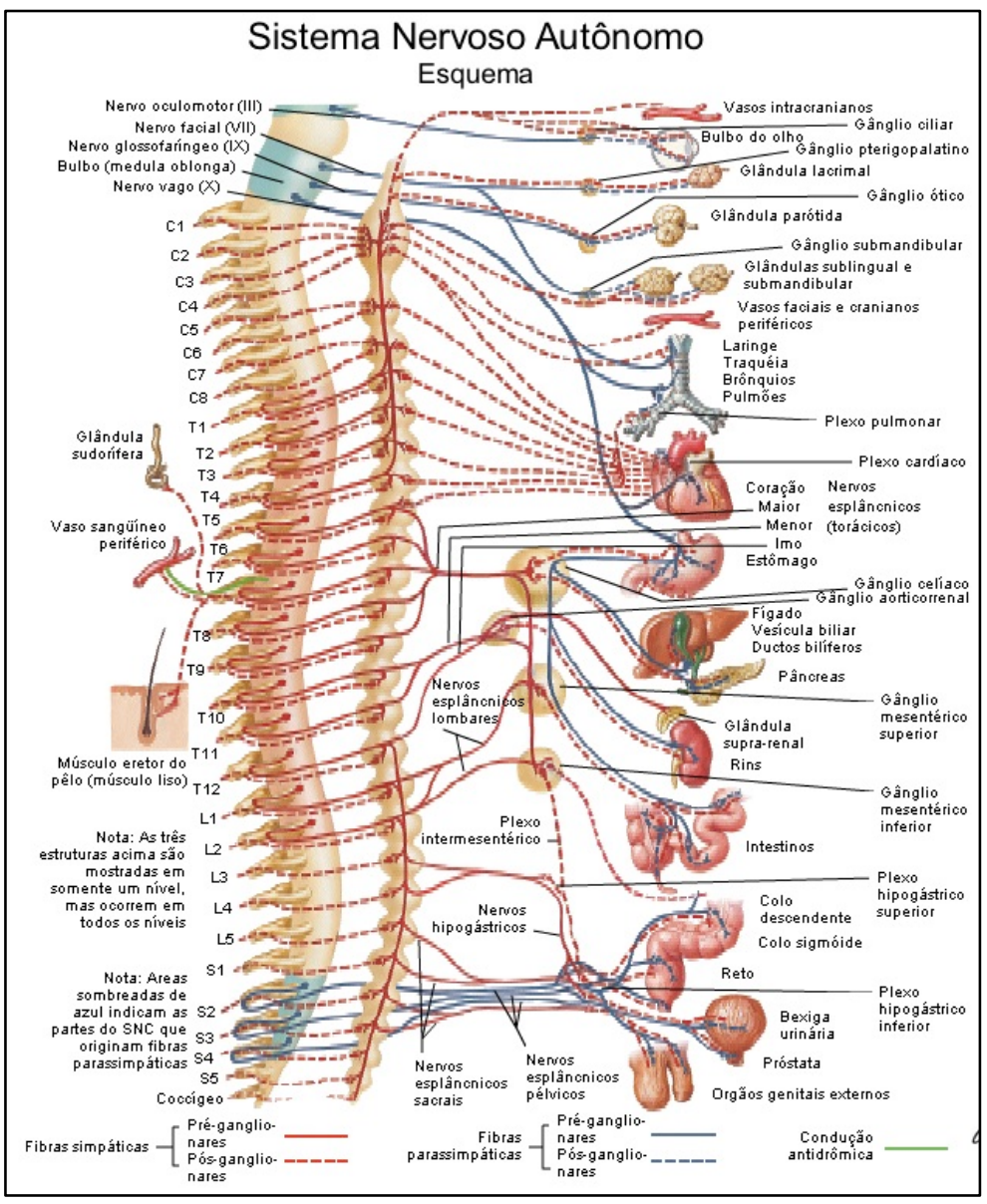

Figura 5. Esquema representativo do sistema nervoso autônomo simpático e parassimpático.

Fonte: Netter, 2011.

\subsubsection{SISTEMA NERVOSO SIMPÁTICO (SNS)}

Foi descrito pela primeira vez por Jacob B Winslow, da sua obra exposição anatômica da estrutura do corpo humano, publicado em 1732, o termo simpático foi baseado de uma palavra grega denominada simpatein, que significa "sofrer com", visto que esse anatomista entendia que as doenças eram levadas de um órgão a outro por meio desse sistema. O SNS foi descrito antes mesmo do entendimento do SNS como parte do SNA (60).

O SNS está anatomicamente localizado no segmento tóraco lombar. Entre os níveis de T1 e L2(3) estão localizados os núcleos intermédios laterais, localizados bilateralmente na substância cinzenta da medula. Tais núcleos são compostos de neurônios pré-sinápticos do SNS e parecem hastes laterais da substância cinzenta, que 
adota forma em $\mathrm{H}$ nos cortes horizontais. Nesses núcleos estão localizados o corpo celular do primeiro neurônio do SNS. O corpo celular do segundo neurônio encontrase em dois lugares, os gânglios para vertebrais e os gânglios pré-vertebrais (54).

Os gânglios para vertebrais se unem para formar as cadeias latero vertebrais simpáticas direita e esquerda de cada lado da coluna vertebral se localizando em toda a extensão da coluna. Cada nível da coluna apresentará um par de gânglio correspondente. Entretanto, na coluna cervical, estes gânglios têm diferenciações, sendo chamados de gânglios cervicais superior, médio e inferior. Para alguns autores o gânglio cervical inferior e o primeiro gânglio torácico se unem formando o gânglio estrelado. $\mathrm{O}$ gânglio ímpar se forma na parte inferior quando se unem os troncos a altura do cóccix (61).

Os gânglios pré-vertebrais se unem em plexos que acompanham os principais ramos da aorta abdominal. Os principais são o gânglio celíaco, mesentérico superior, aórtico renal, mesentérico inferior e Inter mesentérico que farão a inervação das vísceras subdiafragmáticas incluindo estômago, pâncreas, duodeno, fígado, intestino delgado, porção cólon ascendente e $2 / 3$ proximais do cólon transverso e baço celíaco; 1/3 distal do cólon transverso, cólon descendente, sigmóide, reto e ânus mesentérico superior; útero/próstata, bexiga, 1/3 distal do ureter, uretra, ovários/testículos (mesentérico inferior); rins e 2/3 proximais do ureter (aórtico renal) $(61)$.

A partir dessas ramificações, formam-se os plexos autonômicos, que serão denominados, celíaco, mesentérico superior, aórtico renal e mesentérico inferior, sendo que os 3 primeiros com o nervo vago fazendo a porção parassimpática e o último sendo o parassimpático sacral com o nervo esplânico pélvico, respeitando as inervações já mencionadas em relação aos seus órgãos alvo (62).

\subsubsection{SISTEMA NERVOSO PARASSIMPÁTICO (SNP)}

A divisão parassimpática do SNA foi descrita pelo anatomista inglês John Newport Langley, o mesmo que descreveu o SNA, e nomeou o parassimpático por este termo representar "ao lado" do sistema simpático, visto que este sistema se localiza, anatomicamente, no crânio e no sacro (61). Dividido em parassimpático sacral, cranial e visceral, é o antagônico natural do simpático e possui 
anatomia diferenciada. A sua porção cranial é composta pelos pares cranianos III, VII e IX sendo responsável pela porção parassimpática da pupila, secreção lacrimal, secreção nasal e secreção bucal (61).

O nervo vago $(\mathrm{X})$ possui o trajeto mais longo e a distribuição mais extensa de todos os pares cranianos. Seu nome é originado do latim vagari, que significa errante, devido a sua extenso comprimento. Origina-se por uma série de radículas da face lateral do bulbo, núcleo dorsal do vago e núcleo do trato solitário de cada lado do bulbo, que se fundem e deixa o crânio através do forame jugular entre os pares IX e XI $(55,61,62)$. Antes da saída do X do crânio existe um gânglio (Gânglio superior) que está relacionado com o seu componente sensitivo geral do nervo, inferiormente ao forame, há um gânglio inferior (nodoso) relacionado com os componentes sensitivos viscerais do nervo. Continua seu trajeto inferiormente na bainha carotídea até a base do pescoço onde enviará ramos para o palato, a faringe e a laringe $(55,61,62)$.

Em sua porção craniana o $\mathrm{X}$ apresenta um ramo meníngeo para a duramater e um ramo auricular, na região cervical ramos faríngeos para o plexo faríngeo (motor) ramos cardíacos cervicais (aferentes viscerais), nervo laríngeo superior, ramos internos e nervo laríngeo recorrente direito. Na porção torácica o nervo laríngeo recorrente do lado esquerdo, ramos cardíacos torácicos ramos pulmonares e plexo esofágico. Na região abdominal ramos esofágicos, gástricos, hepáticos, celíacos, pilóricos, renais e intestinais para flexura esquerda do cólon. $\mathrm{O}$ nervo esplânico pélvico é o responsável parassimpático dos órgãos da pelve baixa no homem e na mulher originários de S2 a S4 $(55,61,62)$.

Existe uma descrição anatomo-fisiológica que propõe que existe uma porção parassimpática localizada na medula espinhal ignorada pela maioria dos autores. Essa existência é discutida e foi estudada por Hashimoto, Kuré e Okinaka em estudo publicado em 1933 que verificou a presença de fibras vasodilatadoras da língua em segmentos da coluna cervical alta. Seus axônios saem pela raiz posterior para se articular ou no gânglio espinal ou no gânglio laterovertebral com um segundo neurônio homólogo ao neurônio pós ganglionar clássico. Esse sistema parassimpático axial é negado pela maioria dos autores. Atribuiria-se a este sistema parassimpático axial a vasodilatação ativa dos vasos do tronco e dos membros (63). 


\subsubsection{FISIOLOGIA DO SISTEMA NERVOSO AUTÔNOMO}

CARDIOVASCULAR

O SNA controla tônica e reflexamente o sistema cardiovascular, por meio dos neurotransmissores adrenalina e acetilcolina liberadas no coração que modificam o débito cardíaco por alterar a força de contração das fibras miocárdicas e a sua frequência cardíaca. Nos vasos, a liberação de noradrenalina modifica o estado contrátil do músculo liso vascular e a sua resistência vascular periférica. Contudo, o simpático pode exercer efeito nas células musculares lisas e miocárdicas gerando um efeito trófico (20).

As respostas reflexas do SNS e do SNP permitem ajustes do débito cardíaco e da resistência vascular periférica, contribuindo para a estabilização e manutenção da pressão arterial sistêmica durante diferentes situações fisiológicas. Nesse contexto, sabe-se de pelos menos três arcos reflexos que estão envolvidos na modulação da atividade parassimpática para o coração e simpática para coração e vasos, ligados aos baroceptores arteriais (alta pressão), aos receptores cardiopulmonares (baixa pressão) e aos quimoceptores arteriais $(64,65)$.

Os baroceptores arteriais são sensíveis às deformações da parede vascular e, devido ao seu alto ganho, constituem-se na forma mais importante de controle da pressão arterial em curto prazo, ou seja, momento a momento. Além do controle reflexo da atividade autonômica, exercem também um controle tônico inibindo a atividade simpática e, por consequência, estimulando a parassimpática. Assim, o comprometimento de sua função, por não modular a atividade simpática e parassimpática de modo adequado, pode atuar como elemento permissivo ao estabelecimento de alterações primárias de controle da função cardiovascular $(66,67)$.

Os receptores cardiopulmonares são distribuídos em três grupos de receptores que são ativados por mudanças na pressão das câmaras cardíacas, induzindo respostas reflexas autonômicas que modulam principalmente a frequência cardíaca, a dilatação da vasculatura muscular esquelética, a resistência renal e seu débito urinário. Os quimiorreceptores arteriais respondem a aumentos ou quedas de $\mathrm{PO}_{2}, \mathrm{PCO}_{2}$ e/ou pH desencadeando respostas homeostásticas do sistema cardiorrespiratório para corrigir essas variações; quedas na $\mathrm{PO}_{2}$ e aumentos da $\mathrm{PCO}_{2}$ e no $\mathrm{pH}$, elicitam respostas autonômicas reflexas de aumento da resistência periférica $(68,69)$. 
O controle reflexo da circulação comandado pelos baroceptores é um importante preditor de risco após evento cardiovascular. Existem evidências clínicas do valor prognóstico da sensibilidade do barorreflexo e da variabilidade da frequência cardíaca (VFC) na mortalidade cardíaca pós infarto do miocárdio, independente da fração de ejeção do ventrículo esquerdo e de arritmias ventriculares. Dessa forma, intervenções no sentido de melhorar a sensibilidade do barorreflexo, inibir a simpaticotonia e aumentar ou melhorar a participação do imput vagal cardíaco no controle da PA e da FC são estratégias não prioritárias no manejo das doenças cardiovasculares (70).

\subsubsection{DISFUNÇÃO AUTÔNOMA NA INSUFICIÊNCIA CARDÍACA}

A ativação dos sistemas neuro-humorais, especialmente o sistema nervoso simpático, está envolvida na progressão e aumento da mortalidade na IC. No início da lesão miocárdica há a ativação aguda desse sistema como resposta adaptativa e tem por finalidade manter ou restaurar os níveis de débito cardíaco e PA. Em modelos experimentais de IC, a atividade nervosa simpática está presente no início da disfunção ventricular esquerda. Além disto, existem evidências de que alterações precoces do barorreflexo estão relacionadas ao estresse oxidativo, em um modelo experimental de IC $(65,71)$.

Em pacientes com insuficiência cardíaca, Grassi e cols. observaram por meio da técnica de microneurografia, que a atividade nervosa simpática muscular encontrava-se significativamente maior quando comparada aos indivíduos saudáveis, e que ela aumentava com a gravidade da doença. Pacientes com IC de diferentes etiologias sem sobrecarga de volume ou pressão, também demonstraram simpaticotonia importante (72).

Entretanto, o mecanismo essencial responsável pela hiperatividade simpática na IC permanece desconhecido e pode estar relacionado a diversos sistemas, tais como: atenuação da sensibilidade dos mecanorreceptores arteriais e cardíacos; alteração pressórica na artéria e no capilar pulmonar; exacerbação do quimiorreflexo periférico e central; ativação das aferências simpáticas cardíacas que estão relacionadas à sensação de dor cardíaca durante a isquemia coronariana; ativação dos aferentes renais sensíveis a estímulos mecânicos ou químicos e ativação do sistema renina-angiotensina, entre outros tal como a hiperatividade medular simpática 
responsável pelas disfunções osteopáticas. Como a atividade simpática está envolvida na adaptação circulatória da IC desde a instalação e durante a sua progressão e tem valor prognóstico, atualmente são estudadas várias estratégias para reverter o remodelamento miocárdico estrutural e hipertrófico que em geral são divididas em terapias farmacológicas e não farmacológicas $(68,73)$.

Com o tratamento clínico ou cirúrgico adequado na IC, ocorre redução dos níveis circulantes de catecolaminas e da atividade simpática. Em estudo realizado a reversão da disfunção sistólica na miocardiopatia hipertensiva, por tratamento medicamentoso, leva a redução da atividade simpática para o coração e melhora do reflexo cardiopulmonar e do barorreflexo. Estímulos elétricos podem levar a diminuição da simpaticotonia como demonstrado por Vieira e cols com a aplicação de TENS no gânglio estrelado levando a melhora da FC, diminuição da atividade simpática e melhora da PA (74-76).

Existe consenso de que a função vagal preservada é benéfica na manutenção da variabilidade da pressão arterial com consequente proteção de lesão de órgãosalvo, em especial na isquemia do miocárdio. Além disso, existem evidências experimentais de atenuação de resposta da freqüência cardíaca durante a estimulação do nervo vago em cães com insuficiência cardíaca, que podem resultar de alterações na liberação, degradação e ligação da acetilcolina ou na atividade da enzima acetilcolinesterase no coração (77).

A administração de algumas drogas parassimpatomiméticas e betabloqueadoras após infarto do miocárdio e na insuficiência cardíaca tem demonstrado aumento da VFC, indicando efeito protetor sobre o sistema cardiovascular. A busca por terapias com ação cardioprotetora devem ser capazes de inibir a ocorrência de eventos cardíacos em situações de estresse ou esforço físico. A terapia medicamentosa e a atividade física hoje são as terapias de maior relevância clínica para esses pacientes (78-80).

\subsubsection{VARIABILIDADE DA FREQUÊNCIA CARDÍACA (VFC)}

A variabilidade da frequência cardíaca (VFC) demonstra as oscilações entre os batimentos cardíacos consecutivos entre os picos R-R que tem influência do SNA sobre o nódulo sinusal do coração. Descrita e estudada há vários anos, seu interesse 
clínico surgiu quando pode monitorar o sofrimento fetal. Em seguida vieram os estudos associando a VFC diminuída a maior risco de mortalidade pós infarto do miocárdio, sendo assim um preditor de mortalidade. O estudo de Cole et al., do new england journal of medicine demonstrou de vez a associação entre a baixa frequência cardíaca de recuperação pós exercício e a mortalidade. $(75,81,82)$.

\subsubsection{FISIOLOGIA DA VARIABILIDADE DA FREQUÊNCIA}

\section{CARDÍACA}

A excitação cardíaca inicia-se com um impulso gerado no nódulo sinusal, o qual é distribuído pelos átrios, resultando na despolarização atrial, que é representada no ECG pela onda P. Este impulso é conduzido aos ventrículos por meio do nódulo atrioventricular e distribuído pelas fibras de Purkinje, resultando na despolarização dos ventrículos, a qual no ECG é representada pelas ondas $\mathrm{Q}, \mathrm{R}$ e $\mathrm{S}$, formando o complexo QRS. A repolarização ventricular é representada pela onda T. Os índices de VFC são obtidos pela análise dos intervalos entre as ondas $\mathrm{R}$, as quais podem ser captadas por instrumentos como eletrocardiógrafos, conversores analógicos digitais e os cardiofrequencímetros, a partir de sensores externos colocados em pontos específicos do tórax.

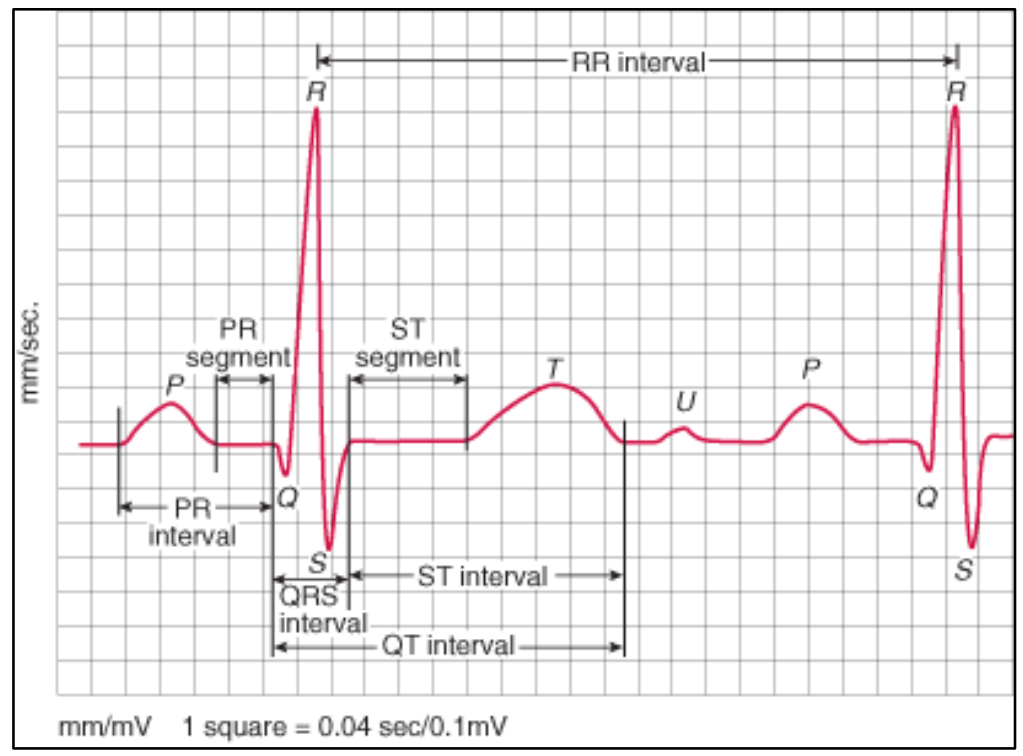

Figura 6. Traçado do eletrocardiograma e o intervalo R-R. 
Fonte:

Site

da

internet:

http://www.medicine.mcgill.ca/physio/vlab/cardio/introecg.htm. Acessado em 10/07/2015.

\subsubsection{DISPOSITIVOS DE MENSURAÇÃO E ÍNDICES DA VARIABILIDADE DA FREQUÊNCIA CARDÍACA}

O padrão ouro para a mensuração da VFC é o ECG. Contudo, apresentam dificuldades de serem aplicados em situações externas ao ambiente de laboratório (atividade física, hospital, clínicas etc). Além disso, apresentam um alto custo, portanto os cardio frequencímetros surgiram para solucionar tais dificuldades. $\mathrm{O}$ frequencímetro Polar S810 apresentou boa acurácia em situações de repouso e de exercício quando comparados com o ECG (83-85). Contudo, o polar não registra possíveis erros de medidas, sendo necessário um ECG prévio para identificar possíveis arritmias. Além disso, é dito que o polar possa superestimar os valores de VFC (86).

A VFC pode ser obtida por meio de vários métodos. Dentre eles, existem os lineares e não lineares. Os métodos lineares são divididos em temporal (realizado por meio de índices estatísticos e geométricos) e frequencial. As unidades temporais são em milissegundos medidos a cada intervalo RR. Em seguida, pode-se obter média, desvio padrão e índices derivados do histograma e mesmo mapa de coordenada cartesianas desse intervalo. A transformação dos valores temporais em frequenciais do intervalo RR determina a análise frequencial (87).

Dentre inúmeras formas de mensurar as medidas temporais, os principais índices são apresentados: Intervalo RR - intervalo entre as medidas R e R do eletrocardiograma, expresso em ms; SDNN - Desvio padrão de todos os intervalos RR normais gravados em um intervalo de tempo, expresso em ms; RMSSD - É a raiz quadrada da média do quadrado das diferenças entre intervalos RR normais adjacentes, em um intervalo de tempo, expresso em ms. O índice SDNN representa atividades simpática e parassimpática sem distinguir as alterações. Entretanto, o índices RMSSD representa a atividade parassimpática (88-90).

As medidas de Poincaré apresentam um método geométrico para análise da dinâmica da VFC. Esta medida representa uma série temporal dentro de um plano 
cartesiano no qual cada intervalo RR é correlacionado com o intervalo antecedente. Com essa definição em um ponto, isso é plotado em um gráfico. Contudo, as mensurações podem ser feitas de forma qualitativa (visual), ou por meio da avaliação da figura formada pelo seu atrator. No ajuste da elipse obtêm-se os índices SD1 e SD2. O SD1 representa a dispersão dos pontos perpendiculares à linha de identidade e parece ser um índice de registro instantâneo da variabilidade batimento a batimento e o SD2 representa a dispersão dos pontos ao longo da linha de identidade e representa a VFC em registros de longa duração $(91,92)$.

Outro método linear muito utilizado é o domínio da frequência. O tacograma contém um sinal aparentemente periódico que oscila no tempo e que é processado por algoritmos matemáticos, como a transformada rápida de Fourier (FFT) ou modelos auto-regressivos (AR). Esta análise decompõe a VFC em componentes oscilatórios fundamentais, sendo que os principais são: Componente de alta frequência (High Frequency - HF), com variação de 0,15 a $0,4 \mathrm{~Hz}$, que corresponde à modulação respiratória e é um indicador da atuação do nervo vago sobre o coração; Componente de baixa frequência (Low Frequency - LF), com variação entre 0,04 e 0,15Hz, que é decorrente da ação conjunta dos componentes vagal e simpático sobre o coração, com predominância do simpático e suas respectivas medidas normalizadas. A relação LF/HF caracteriza o balanço simpato-vagal sobre o coração. O poder total (total power) representa a área do gráfico do tacógrafo. Outras medidas como a VLF e ULF (very low frequency e ultra low frequency respectivamente) são questionadas quanto a sua função $(87,88,91,93,94)$. A tabela 1 resume as principais variáveis da VFC. 


\begin{tabular}{|c|c|c|}
\hline $\begin{array}{l}\text { Variáveis } \\
\text { Temporais }\end{array}$ & $\begin{array}{l}\text { Variáveis } \\
\text { Frequenciais }\end{array}$ & Análise de Poincarré \\
\hline $\begin{array}{l}\text { Média do Intervalo RR - valor } \\
\text { dado em Milissegundos (ms). } \\
\text { Indicador vagal }\end{array}$ & $\begin{array}{l}\text { TP - Total Power. Área } \\
\text { espectral total (PT) - } \\
\text { indicador global do sistema }\end{array}$ & $\begin{array}{l}\text { SD1 Desvio vertical (ms) - } \\
\text { indicador vagal }\end{array}$ \\
\hline $\begin{array}{l}\text { SDNN - Standard Deviation } \\
\text { N-N (ms). Desvio Padrão dos } \\
\text { intervalos N-N. Indicador } \\
\text { vagal }\end{array}$ & $\begin{array}{l}\text { LF - Low Frequency. } \\
\text { Baixa frequência (BF) } \\
(\mathrm{ms} 2) \text {, espectral absoluta de } \\
\text { BF - indicador } \\
\text { simpatovagal }\end{array}$ & $\begin{array}{l}\text { SD2 Desvio horizontal (ms) } \\
\text { - indicador global } \\
\text { simpatovagal }\end{array}$ \\
\hline \multirow[t]{4}{*}{$\begin{array}{l}\text { RMSSD - Rooth Mean Square } \\
\text { Standard Deviation (ms). } \\
\text { Desvio padrão da Raíz } \\
\text { quadrada da média. Indicador } \\
\text { vagal }\end{array}$} & $\begin{array}{l}\text { LFnorm. - Baixa freqüência } \\
\text { normalizada (BFnorm) }(\%) \text {, } \\
\text { espectral normatizada de BF } \\
\text { - percentual de simpático }\end{array}$ & \\
\hline & $\begin{array}{l}\text { HF - High Frequency. Alta } \\
\text { freqüência (AF) ( ms } 2) \text {, } \\
\text { espectral absoluta de AF - } \\
\text { indicador vagal }\end{array}$ & \\
\hline & $\begin{array}{l}\text { HFnorm. - Alta freqüência } \\
\text { normalizada (AFnorm), } \\
\text { espectral normatizada de } \\
\text { AF }(\%) \text { - percentual vagal }\end{array}$ & \\
\hline & $\begin{array}{l}\mathrm{LF} / \mathrm{HF} \text { - Balanço } \\
\text { autonômico (BF/AF) ( ms } 2 / \\
\mathrm{ms} 2) \text { - índice entre } \\
\text { simpático e vago, sendo que } \\
\text { os valores }>1 \text { indicam } \\
\text { simpaticotonia relativa, }=1 \\
\text { anfotônico, }<1 \text { vagotonia } \\
\text { relativa. }\end{array}$ & \\
\hline
\end{tabular}

Tabela 2. Variáveis desfechos da VFC e suas explicações.

Fonte: modificado de Vanderlei, 2009. 


\subsection{JUSTIFICATIVA}

Portanto, ainda há escassez de estudos avaliando os efeitos do tratamento manipulativo osteopático na função vascular e autonômica ao mesmo tempo em pacientes com IC (lacuna 1). Soma-se a isso as controversas respostas autonômicas pós técnicas de manipulação (AVBA) ou técnicas de mobilização intra articular (MIA) da coluna vertebral em indivíduos saudáveis (lacuna 2). E por último, dúvida acerca das respostas cardiovasculares da pressão arterial e frequência cardíaca pós tratamento manipulativo osteopático cervical do tipo manipulação de AVBA (lacuna $3)$.

Nesta tese são apresentados 3 estudos desenvolvidos para se preencher as lacunas descritas. Realizou-se um ensaio clínico randomizado e aleatorizado [manuscrito 1] com o objetivo de verificar as modulações vasculares e autonômicas após o tratamento manipulativo osteopático em cardiopatas (lacuna 1). Uma revisão sistemática com metanálise [manuscrito 2] para tentar entender os feitos da manipulação da coluna no SNA (lacuna 2), além de outro ensaio clínico randomizado e aleatorizado [manuscrito 3] acerca dos efeitos do tratamento manipulativo osteopático cervical nas variáveis cardiovasculares em saudáveis checando a segurança dos pacientes que recebem as técnicas de AVBA (lacuna 3).

\subsection{OBJETIVOS PROPOSTOS}

A presente tese possui como objetivo principal: verificar os efeitos do tratamento manipulativo osteopático na função vascular e autonômica de pacientes cardiopatas por meio de um ensaio clínico randomizado (lacuna 1). Como segundo objetivo verificar o estado da arte da literatura em relação as manipulações de alta velocidade e baixa amplitude (AVBA) e a mobilização intra articular (MIA) ambas na coluna vertebral e as modulações do SNA e, ainda comparar os resultados dos trabalhos encontrados dentro deste tema por meio de uma revisão sistemática com metanálise (lacuna 2). E por último, demonstrar quais seriam as respostas do tratamento manipulativo osteopático cervical na PA e FC em indivíduos saudáveis por meio de um segundo ensaio clínico randomizado, (lacuna 3). 


\subsubsection{ORGANIZAÇÃO DO TRABALHO}

A organização da presente tese é apresentada da seguinte maneira:

1 - introdução e contextualização dos temas que apresentam relação com a tese, justificativa e objetivos propostos da tese.

2 - apresentação de 3 resumos dos manuscritos que envolvem os trabalhos durante o doutorado.

3 - discussão geral e conclusões integrando as partes do projeto e detalhes extras da execução da tese que não puderam ser apresentados nos manuscritos.

4 - como anexo, uma lista de outros estudos feitos durante o programa de doutorado, que foram apresentados em congressos e auxiliou a um racional mais completo para essa escrita deste tese. 


\section{ARTIGOS}

Foram executados três manuscritos para constar o corpo da presente tese. o primeiro intitulado: efeitos do tratamento manipulativo osteopático na função vascular e autonômica em cardiopatas: um ensaio clínico randomizado é denominado manuscrito 1. o segundo foi chamado de: Efeitos da manipulação e mobilização da coluna vertebral no sistema nervoso autônomo: uma revisão sistemática com metanálise. E por fim, o último manuscrito denomina-se: $\mathrm{O}$ tratamento manipulativo osteopático cervical tem efeito imediato e tardio nas variáveis cardiovasculares em indivíduos saudáveis?

\subsection{MANUSCRITO 1}

O manuscrito 1 será submetido para a revista Journal of Physioterapy e foi redigido de acordo com suas normas. Entretanto, com a finalidade de evitar o autoplágio, é apresentado o resumo desse manuscrito.

\section{RESUMO}

Objetivo: Verificar o efeito agudo e time course do tratamento manipulativo osteopático (TMO) na função vascular e autonômica.

Desenho: Ensaio clinico randomizado. Pacientes foram divididos em grupo TMO e Sham.

Parâmetros: Reabilitação Cardiorrespiratória e Tecnologias Assistivas em Fisioterapia

Participantes: Pacientes com insuficiência cardíaca ( $\mathrm{n}=20$, idade 59,5 $\pm 11,82$, Weber Class C).

Intervenções: Uma sessão de TMO e Sham com um aparelho chamado ativador quiroprático sem carga.

Mensuração da medida principal: Medimos a função vascular por meio da dilatação medida pelo fluxo da artéria braquial (DMF) e a função autonômica por meio da variabilidade da frequência cardíaca. As medidas foram realizadas Pre 
intervenção, Pós e time course de 10 minutos. Para fins de comparação foi utilizada a análise entre grupos multivariada (MANOVA).

Resultados: Verificamos um aumento da DMF (pre x time course $14 \% \pm 2,29$ para $23,5 \% \pm 6,87 \mathrm{p}=0,027$ ), absolute difference (pre $\mathrm{x}$ time course $0,6 \mathrm{~mm} \pm 0,159$ para $1,07 \pm 0,29 \mathrm{p}=0,03$ ) e peak diammeter (pre $\mathrm{x}$ time course $4,9 \mathrm{~mm} \pm 0,74$ para 5,67 $\pm 0,76 \mathrm{p}=0,003$ and post $\mathrm{x}$ time course $5,2 \mathrm{~mm} \pm 0,58$ para $5,67 \pm 0,76 \mathrm{p}=0,02$ ). Todavia, na função autonômica houve aumento dos índices vagais imediatamente após a técnica (RMSSD, SDNN e SD1) e após 10 minutos de follow-up uma manutenção do aumento vagal além de aumento de índice simpático (LF).

Conclusão: A informação mais importante do nosso estudo foi que o TMO é capaz de modular o sistema vascular e autonômico em pacientes com insuficiência cardíaca. Adicionalmente os ajustes vasculares parecem ja ocorrer mesmo antes da modulação autonômica simpática.

Palavras-chave: tratamento manipulativo osteopático, osteopatia, cardiopata, DMF, doppler, variabilidade de frequência cardíaca, sistema nervosa autonômico.

\subsection{MANUSCRITO 2}

O manuscrito 2 será submetido para a revista Archives of Physical medicine and Rehabilitation e foi redigido de acordo com suas normas. Seu resumo é apresentado a seguir:

\section{Resumo}

O tratamento manual da coluna vertebral (TMCV) atribui como uma de seus principais embasamentos físiológicos, os efeitos secundários as modulações autonômicas que este é capaz de causar, todavia essas respostas ainda são controversas na literatura. O objetivo desta revisão sistemática com metanálise foi avaliar a influencia de duas técnicas distintas de TMCV na modulação do sistema nervoso autonômico (SNA). Nós procuramos em três base de dados, MEDLINE (Fev 1965 - Novembro 2015), PEDro (Physiotherapy evidence database) e na Chochrane 
library (2015) até Novembro de 2015. Verficamos a qualidade metodológica dos estudos pela escala PEDro. Incluimos os ensaios clínicos que utilizaram mensuração do SNA com variabilidade de frequência cardíaca (VFC), frequência cardíaca (FC) ou a condutância da pele (CP) e que utilizaram a técnica de manipulação de alta velocidade de baixa amplitude (AVBA) ou de mobilização intra articular (MIA) como TMCV. Dois avaliadores independentes selecionaram seis estudos que tiveram pontuação na escala PEDro acima de 6 em todas as variáveis estudadas de VFC, FC e CP. As técnicas de AVBA promovem aumento da $\mathrm{LF}_{\text {norm }}\left(5,82 \mathrm{~ms}^{2}\right.$ [95\% CI: 2,26 to 9,38 $\left.\left.\mathrm{I}^{2}=0 \% \mathrm{p}=0,001\right]\right) \mathrm{LF} / \mathrm{HF}\left(0,38\right.$ [95\% CI: 0,10 to $\left.\left.0,66 \mathrm{I}^{2}=0 \% \mathrm{p}=0,008\right]\right)$, enquanto que, durante a aplicação de MIA, há um aumento da CP(6,66 [95\% CI: 1,28 to $\left.\left.12,04 \mathrm{I}^{2}=0 \% \mathrm{p}=0,02\right]\right)$. O local de aplicação da técnica não influenciou o resultado obtido. Em nossos resultados, as técnicas de AVBA não alteraram o sistema nervoso parassimpático e a FC. Supreendentemente, após a execução da MIA, condutância da pele também não alterou. Esta metanálise indica que a TMCV, em especial as manobras de AVBA e de MIA, independente da região da coluna, provocam uma excitação no SNA simpático em sujeitos sadios. Entretanto a mudança provocada pela MIA ocorre apenas durante a execução da técnica, mas parece não se manter após a aplicação.

\subsection{MANUSCRITO 3}

O manuscrito 3 foi submetido para a revista Journal of manipulative and physiological therapeutics e o resumo é apresentado a seguir:

\section{RESUMO}

OBJETIVO: avaliar os efeitos do tratamento manipulativo osteopático (TMO) cervical sobre a pressão arterial (PA) e freqüência cardíaca $(\mathrm{FC})$ de maneiras imediatas e time course em indivíduos jovens saudáveis.

MÉTODOS: Um único ensaio clínico randomizado duplo cego foi realizado. A amostra do estudo incluiu 77 voluntários aleatoriamente randomizados em 3 grupos: grupo tratamento manipulativo osteopático (TMOG) com estimulação sob a região da coluna $\mathrm{C} 1$ e $\mathrm{C} 2$ bilateralmente, o grupo Sham (SHG) com dispositivo denominado 
ativador quiroprático sem carga e grupo controle (CTG) sem estimulação. Foi avaliada a PA e FC no início e após 1 e 10 minutos e 24 horas posteriores. Para comparar a PA e FC entre os grupos de análise multivariada de variância (MANOVA) foi utilizado.

RESULTADOS: Não foi encontrada qualquer alteração relevante na PA e FC após TMO cervical em nenhum dos grupos imediatamente. Além disso, os resultados não demonstram alterações significativas em 24 horas de time course.

CONCLUSÃO: Apesar da proximidade do gânglio cervical com C1 e C2 vértebras, este estudo mostra que o TMO cervical não induz uma alteração imediata e 24 horas significativa na PA e FC. De acordo com estes resultados, os pacientes jovens saudáveis não apresentam efeitos colaterais cardiovasculares com manipulação cervical.

Palavras-chave: tratamento manipulativo osteopático; manipulação Osteopática; manipulação vertebral; manipulação da coluna vertebral; frequência cardíaca; pressão sanguínea 


\section{DISCUSSÃO GERAL E CONCLUSÕES}

\subsection{INTEGRAÇÃO DAS PARTES DO PROJETO}

Para o manuscrito 1, foi demonstrado por meio deste ensaio clínico randomizado que o tratamento manipulativo osteopático é capaz de promover a modulação vascular aumentando a DMF braquial, e suas medidas adjacentes como a medida basal e o diâmetro de pico. Contudo, tal resposta parece não ser modulada agudamente pelo SNA simpático visto que esta foi após a mudança vascular. Do que é do nosso conhecimento, esse é o primeiro estudo que avaliou as respostas vasculares e autônomas ao mesmo tempo após o tratamento manipulativo osteopático. Esse resposta nos leva a entender que as alterações do SNA simpático promovidas pelo tratamento manipulativo osteopático pode ocorrer em resposta à alteração arterial prévia.

O manuscrito 2 demonstra que a compilação dos resultados da presente metaanalise demonstrou que as técnicas manuais da coluna vertebral são capazes de modular de forma aguda o SNA em sujeitos saudáveis em comparação com o sham. As técnicas de alta velocidade e baixa amplitude (AVBA), promovem aumento de 2 variáveis da variabilidade da frequência cardíaca $\left(\mathrm{LF}_{\text {norm }}\right.$ e $\left.\mathrm{LF} / \mathrm{HF}\right)$, enquanto que, durante a aplicação da mobilização intra articular (MIA), há um aumento da condutância da pele (CP). O local de aplicação da técnica não influenciou o resultado obtido. Em nossos resultados, as técnicas de AVBA não alteraram o sistema nervoso parassimpático e a frequência cardíaca. Supreendentemente, após a execução da MIA a CP também não alterou. Do que é do nosso conhecimento, esse é o primeiro estudo que avalia essas respostas por meio da metanálise.

O manuscrito 3 demonstra que os resultados do tratamento manipulativo osteopático cervical não alterou o sistema cardiovascular em jovens saudáveis. Devido à proximidade dos gânglios cervicais simpático na coluna cervical, era esperado que o pudesse modular a FC e PA. Foram avaliados os efeitos imediatos e após 10 minutos porque talvez as respostas cardiovasculares poderiam levar mais tempo para ocorrer. Além disso, esperamos 24 horas para avaliar novamente, no entanto, sem mudanças. Com isso, pode-se concluir que essas técnicas apresentam 
segurança do ponto de vista cardiovascular, visto que não leva a alterações significativas.

Para os dados do manuscrito 1, além das medidas de vasculares de DMF e autonômicas de VFC, foi mensurado o fluxo arterial durante a desoclusão. Considerando a limitação da formatação, este dado não foi incluído no manuscrito 1 , porém é demonstrado nesta seção. O fluxo foi mensurado pré, pos imediato e 10 minutos de prosseguimento. A cada liberação de oclusão foram mensuradas 10 medidas de velocidade média e índice de resistência. A figura 16 mostra o comportamento do fluxo sanguíneo por meio das medidas de velocidade média e índice de resistência do vaso. Há diminuição da velocidade e da resistência, indo de acordo com os achados prévios do manuscrito 1 .
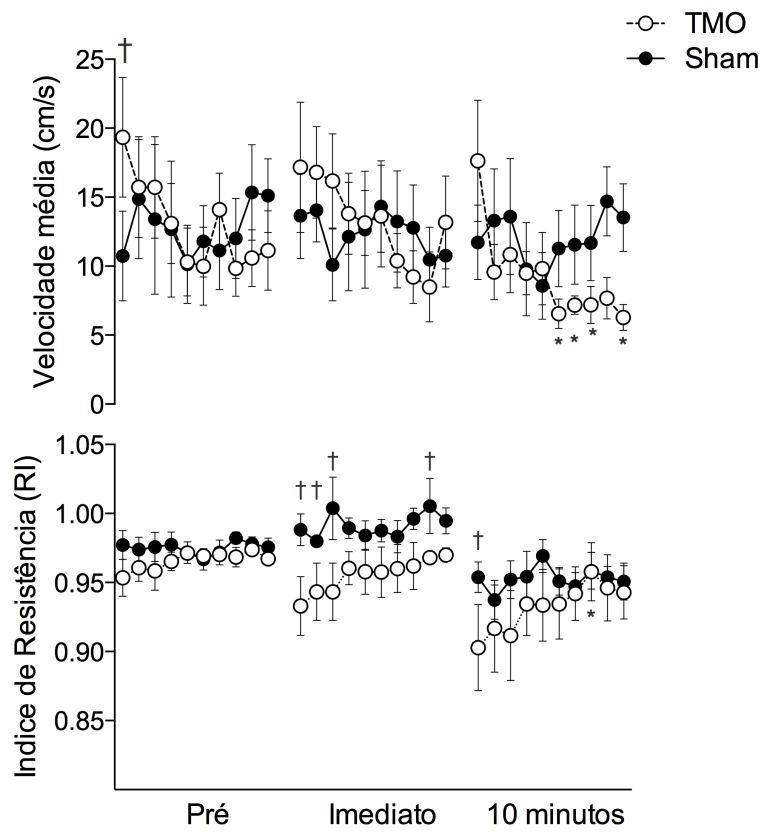

Figura 7. Comportamento da velocidade média do vaso e índice de resistência pré, pós imediato e 10 minutos de prosseguimento. TMO - tratamento manipulativo osteopático

Fonte: próprio autor, 2015.

O tratamento manipulativo osteopático parece de fato modular o sistema vascular e autonômico em cardiopatas. Contudo, o fato que chama mais atenção nesse primeiro estudo é que as respostas autonômicas simpáticas parecem vir depois das 
respostas vasculares. Houve um incremento parassimpático e a modulação vascular ao mesmo tempo, gerando um aumento da resposta da DMF. Dentre as respostas autonômicas que seriam esperadas, a diminuição do SNA simpático justificaria o aumento do diâmetro do vaso, porém isso não ocorreu. Portanto, tal padrão parece nos levar a um entendimento que, nesse caso, as respostas autonômicas simpáticas podem ser moduladas pelo comportamento vascular e/ou central do sistema nervoso.

Em relação ao manuscrito 2, o resultado mostram duas respostas distintas, primeiramente demonstrando que as técnicas de AVBA ativam o SNA simpático. Ainda foi verificado que não altera variáveis cardiovasculares tais como a FC. Entretanto, quando a técncia de MIA é realizada, durante a execução da mesma há a ativação autonômica simpatico, porém quando se deixa de dar o estímulo, a ativação cessa. Tal mecanismo é explicado pela ativação da área cinzenta dorsal periaquedural (DPAG) e, através de vias descendentes neurais, ativando o centro cardioacelerador localizado de T1 a T4 na medula espinhal. Com isso, a mobilização, que tem um estímulo por maior tempo no indivíduo, poderia ativar todo esse trajeto levando não apenas a um aumento do SNA simpático, porém em nossa metanálise, vimos que isso não é duradouro, ao contrário das técnicas de AVBA. Ainda se tem dúvidas em relação a quantidade de tempo em que o estímulo perdura.

Em relação ao manuscrito 3, a resposta do estudo que demonstrou que a PA e FC não alteram frente a tratamento manipulativo osteopático cervical nos indivíduos saudáveis mostra segurança sob o ponto de vista cardiovascular. Na presente tese, verificou-se que, ao mesmo tempo que as técnicas de AVBA promovem a modulação simpatica, elas não alteram as variáveis cardiovasculares. Essa informação merece destaque porque se ativar o sistema simpático sem provocar um aumento da PA ou da FC pode ser mais benéfico às pessoas a dependar da sua condição de saúde.

Quando se relaciona a resposta dos 3 estudos, verifica-se a necessidade de se elucidar melhor os mecanismos da função vascular e autonômica pós tratamento manipulativo osteopático. Encontramos nos estudos respostas que nos leva adiante no sentido de entender que as respostas vasculares parecem ser mais independentes do SNA. O SNA simpático, pode ser influenciado pelas respostas vasculares, além de verificarmos as diferenças entre se realizer a técnica de AVBA e de MIA. Contudo, alguns questionamentos ainda ficam para futuras pesquisas e teses: As respostas em 
pessoas saudáveis ao tratamento manipulativo osteopático seria da mesma forma como o encontrado em cardiopatas? O estímulo mecânico poderia levar a respostas específicas para os receptores autonômicos cardíacos? Em longo prazo, quais seriam as respostas? Ainda a ciência busca essas respostas.

\subsection{DETALHES DA EXECUÇÃO DO PROJETO}

Em termos metodológicos, alguns detalhamentos acerca das técnicas não puderam ser colocados no manuscrito final 1. Além disso, foram realizadas uma série de procedimentos de avaliação de dados que poderiam também ser melhor explicados, além de alguns dados que foram deixados para possíveis discussões posteriores. Esta parte é um complemento dessas informações.

\subsubsection{TRATAMENTO OSTEOPÁTICO}

As manipulações osteopáticas foram realizadas utilizando uma sequência de tratamento baseada na avaliação TART (tensão, assimetria, restrição e dor), conforme descrito no manuscrito 1, porém cabe aqui um maior aprofundamento. Foram verificadas as tensões, assimetrias, restrições de movimento e dor nos pacientes nas regiões relacionadas ao coração e sua inervação autonômica. A base do crânio, coluna cervical e torácica, primeira costela, caixa torácica, diafrágma e a mobilidade cardíaca foram avaliadas sob o ponto de vista osteopático. A maioria das disfunções foram encontradas na cervical média, base do crânio a direita no osso occiptal, primeira costela e na transição C7-T1. Foram corrigidas tais disfunções, associada a um tratamento mais hemodinâmico que incluíram técnicas de bombeio do ducto torácico (thoracic pump) e de descompressão cardíaca. Nenhum paciente relatou efeito adverso durante a terapia. O grupo sham recebeu simulação de terapia por meio do ativador quiroprático que foi realizado nos mesmos locais onde foram encontradas as disfunções. As figuras de 8 a 12 mostram as técnicas empregadas durante o tratamento osteopático de acordo com a avaliação. 

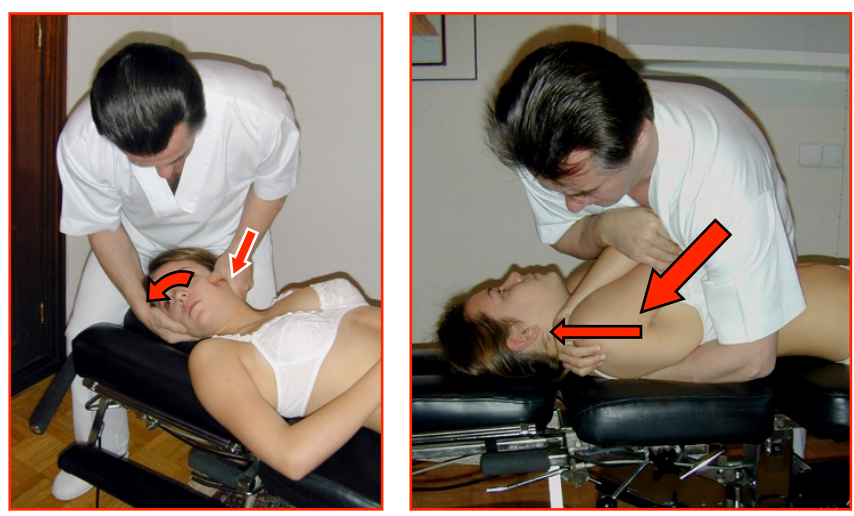

Figura 8. Técnica de manipulação cervical em rotação para a esquerda e manipulação da primeira costela em posterioridade.

Fonte: Ricard, 1993
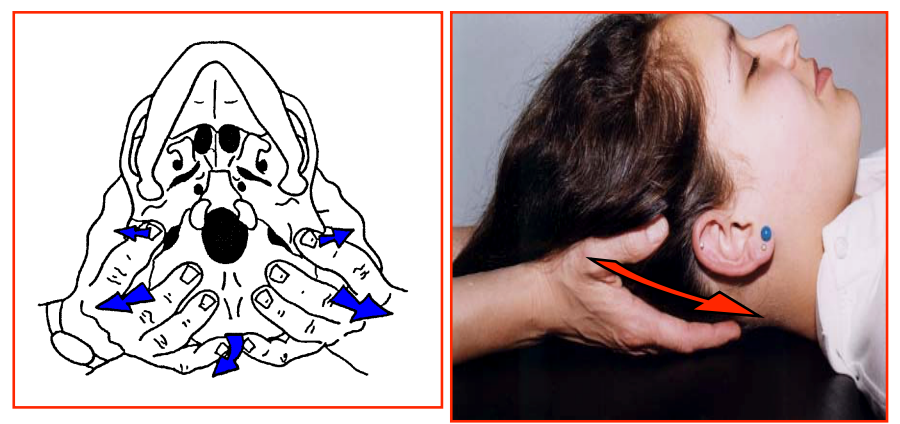

Figura 9. Técnica de descompressão da base do crânio segundo Magoun.

Fonte: Ricard, 2002.

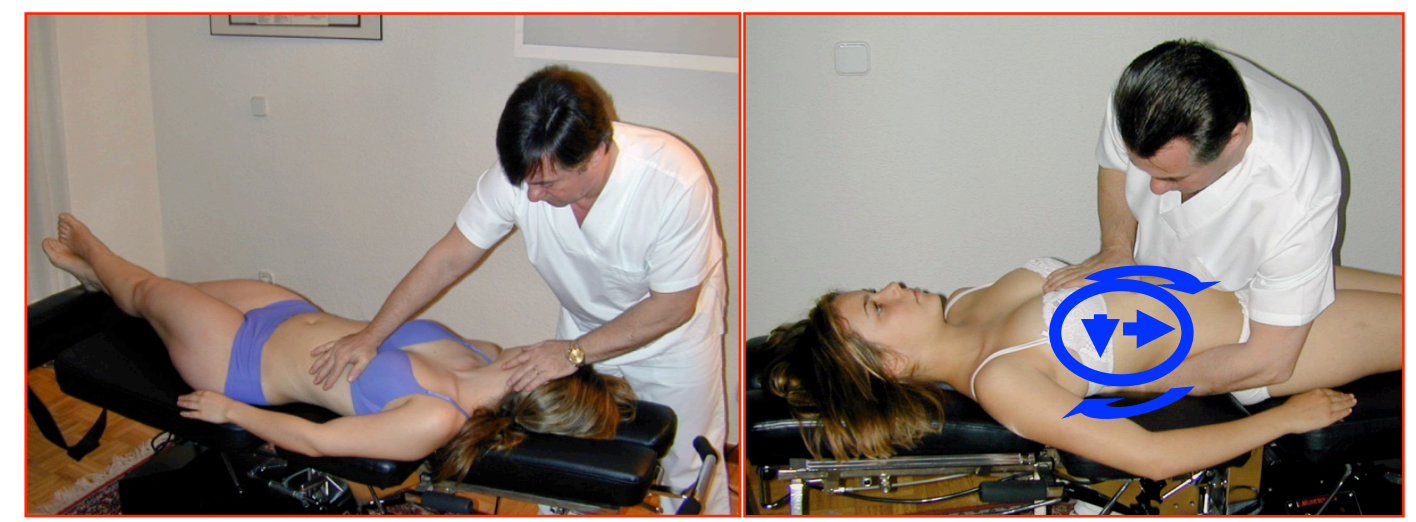

Figura 10. Técnica de estiramento da pleura a esquerda e técnica funcional para o diafragma

Fonte: Ricard, 2015 (25) 


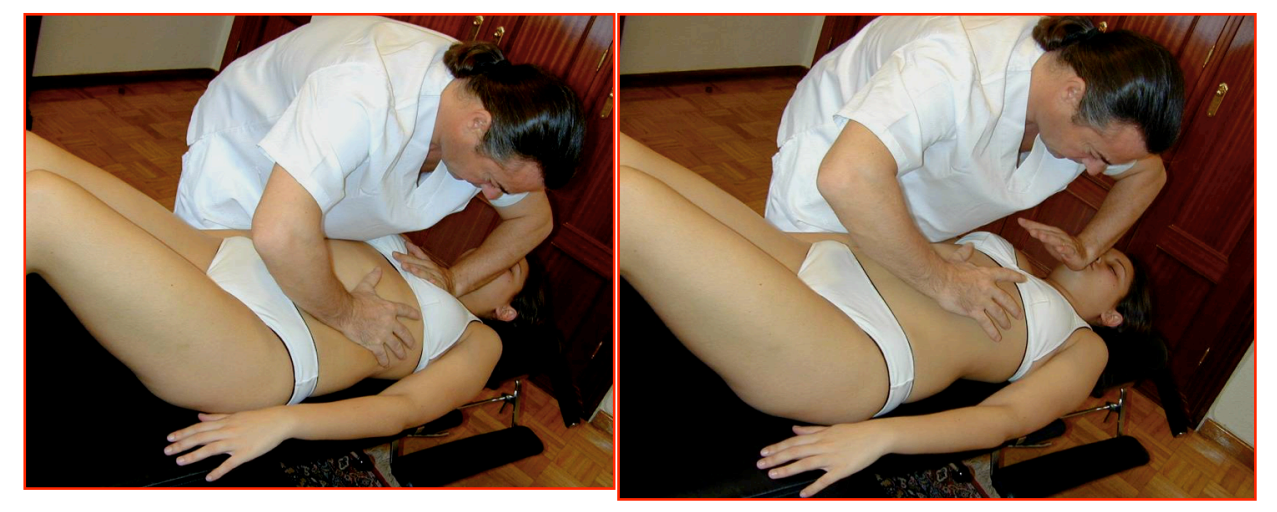

Figura 11. Técnica de descompressão cardíaca. Primeira fase de compressão e segunda fase de descompressão.

Fonte: Ricard, 2015
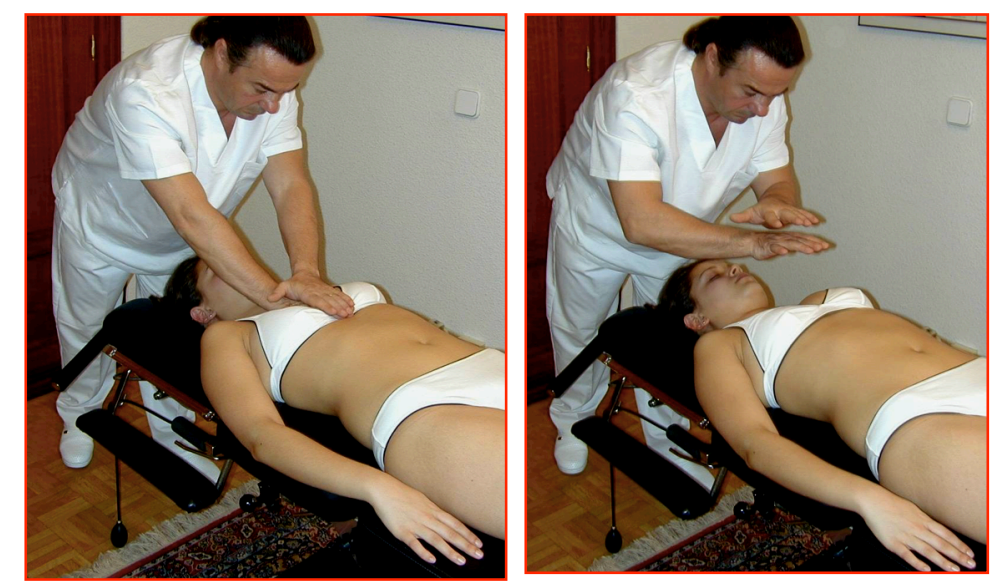

Figura 12. Técnica de bombeio do ducto torácico.

Fonte: Ricard, 2015

\subsubsection{AVALIAÇÃO DOS PACIENTES - COLETA DE DADOS}

As avaliações foram realizadas ao mesmo tempo, porém foram coletadas outras medidas, tais como a VFC antes, durante e logo após o tratamento manipulativo osteopático. Também medidas de algometria pré e pós tratamento manipulativo osteopático, além dos dados de doppler pré, pós imediato e 10 minutos depois nas artérias braquial, carótida comum e femoral. Tais medidas não entraram no estudo principal pois são medidas que nos ajudam a entender outros aspectos fisiológicas das manipulações. De forma geral, o tratamento manipulativo osteopático provocou um aumento das medidas simpáticas e quase nenhuma alteração nos fluxos, 
a não ser da velocidade média do sangue na artéria braquial e uma pequena diferença na femoral.

As figuras em sequência demonstram o protocolo realizado para fins de ilustração. Na figura 13 demonstra o aparelho de ultrassom duplex ao qual foram realizadas as coletas. Em seguida na figura 14 um dos voluntários durante a coleta de dados. As figuras 15, 16 e 17 demonstram os dados já trabalhados de DMF, doppler e VFC.

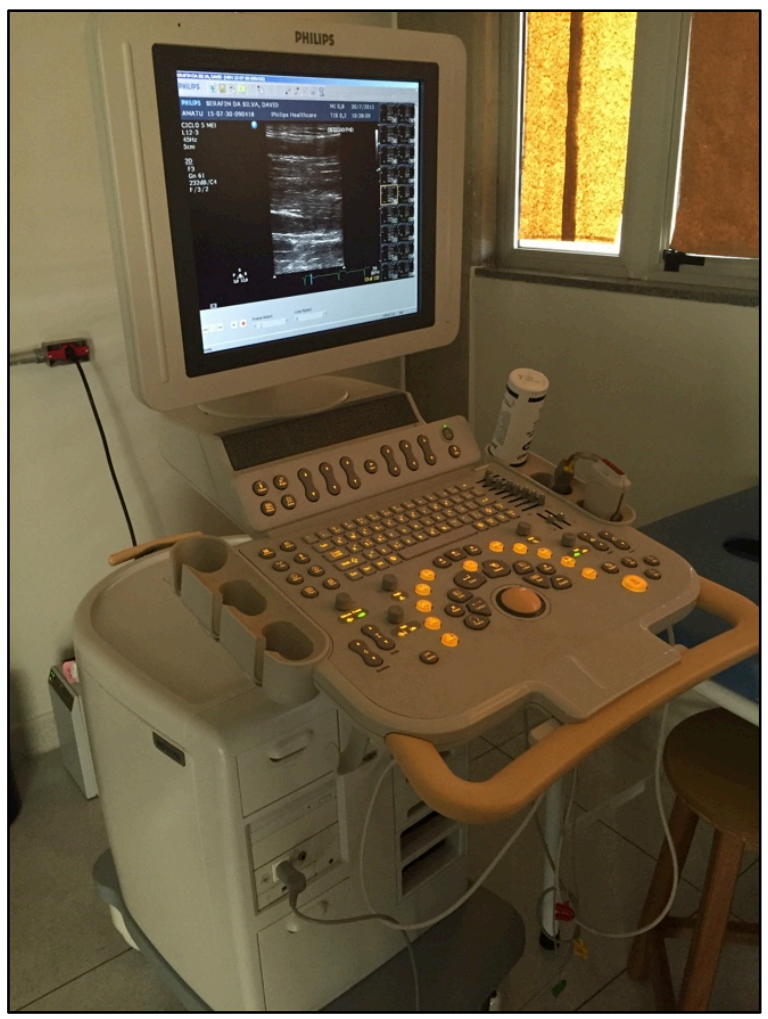

Figura 13. Aparelho de ultrassom utilizado no estudo.

Fonte: próprio autor, 2015.

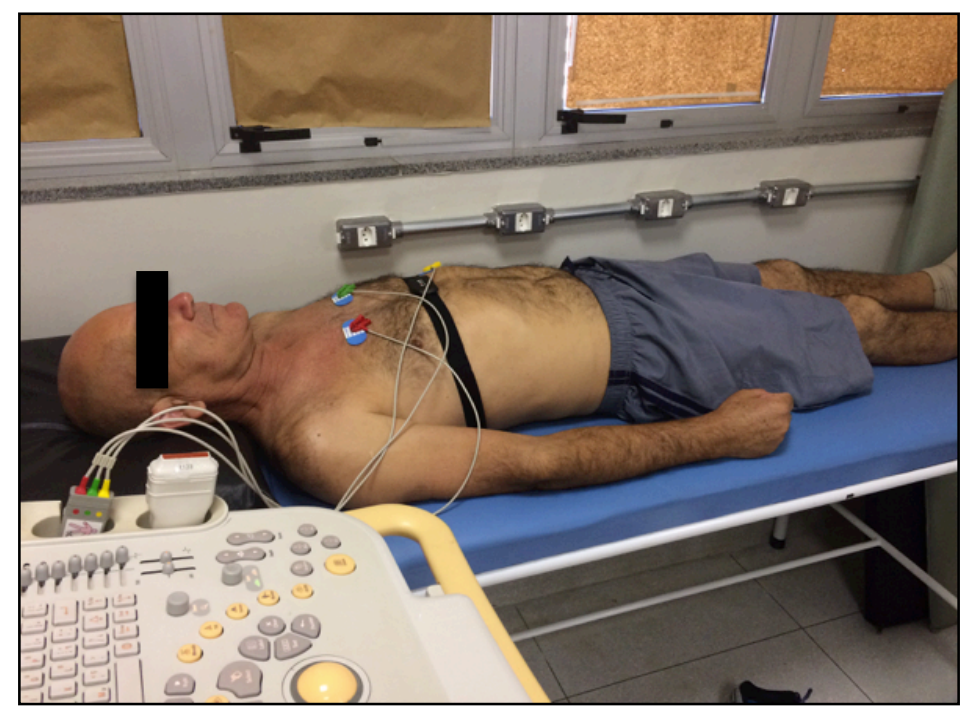


Figura 14. Exemplo de coleta de dados em voluntário

Fonte: próprio autor, 2015.

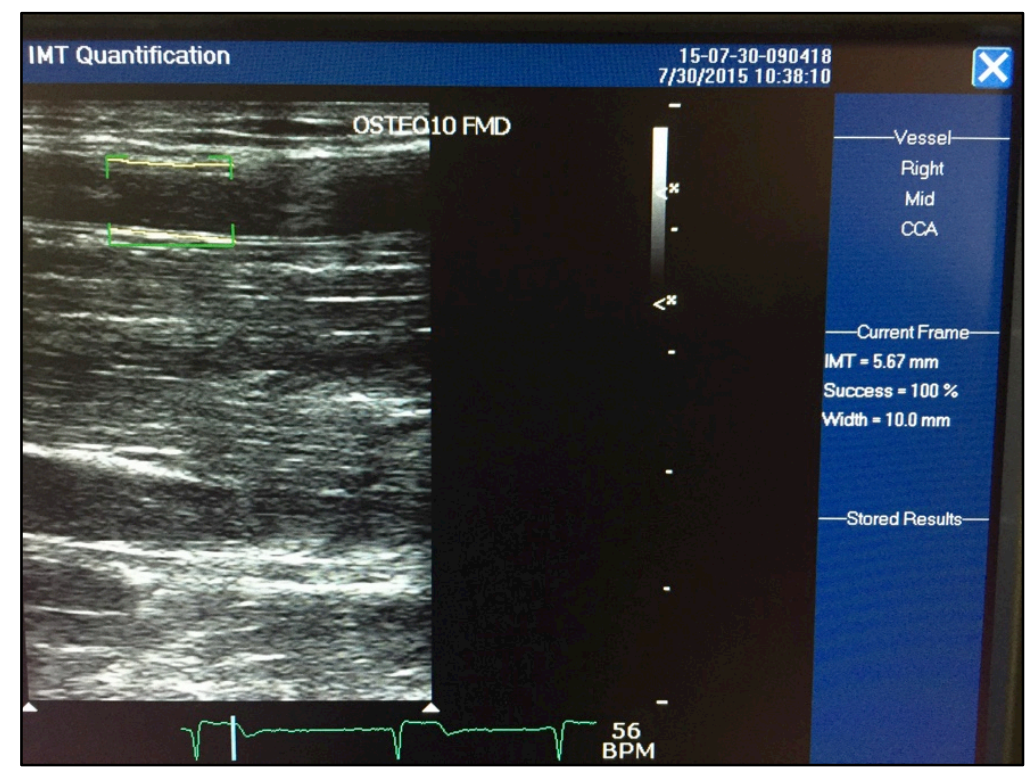

Figura 15. Exemplo de análise com software de detector de borda para a DMF.

Fonte: próprio autor, 2015

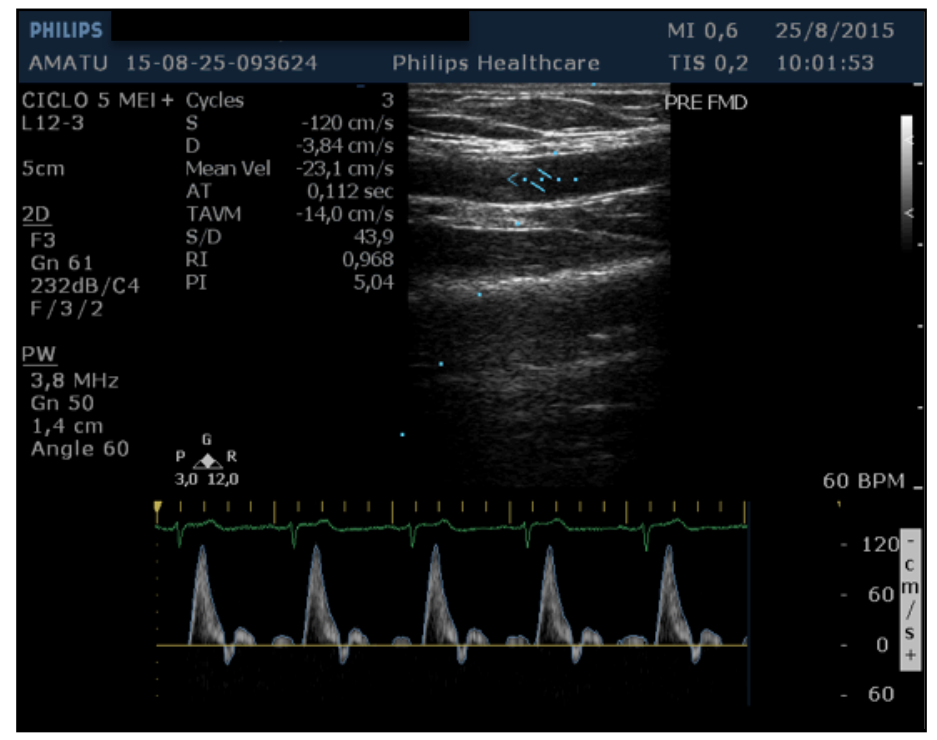

Figura 16. Exemplo de análise de doppler. 
Fonte: próprio autor, 2015

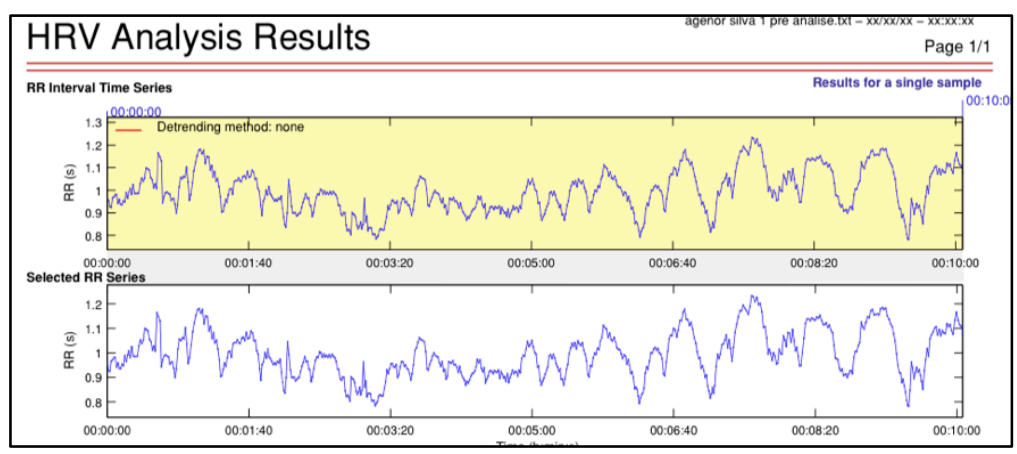

Figura 17. Exemplo de tacograma de variabilidade de frequência cardíaca

Fonte: próprio autor, 2015

\subsection{CONCLUSÕES}

Portanto, segue a nossa conclusão para a lacuna 1: A informação mais importante do nosso estudo foi que o tratamento manipulativo osteopático é capaz de modular o sistema vascular e autonômico em pacientes com insuficiência cardíaca. Adicionalmente os ajustes vasculares parecem já ocorrer mesmo antes da modulação autonômica simpática. Para a lacuna 2: Revisão sistemática com metanálise: Surpreendentemente, os estudos que mensuraram o SNA com a VFC utilizaram a AVBA como TMCV e aqueles que usaram a $\mathrm{CP}$ a técnica empregada foi a MIA. Verificamos a ativação simpática com um aumento na LF normalizada $\left(5,82 \mathrm{~ms}^{2}\right.$ [95\% CI: 2,26 to $\left.\left.9,38 \mathrm{I}^{2}=0 \% \mathrm{p}=0,001\right]\right)$, e $\mathrm{LF} / \mathrm{HF}$ (0,38 [95\% CI: 0,10 to $0,66 \mathrm{I}^{2}=0 \%$ $\mathrm{p}=0,008])$ e aumento da CP $\left(6,66\right.$ [95\% CI: 1,28 to $\left.\left.12,04 \mathrm{I}^{2}=0 \% \mathrm{p}=0,02\right]\right)$ durante a aplicação da MIA. Contudo, não foram evidenciadas mudanças na FC, no sistema nervoso parassimpático e nem na $\mathrm{CP}$ pós MIA. Portanto, o TMCV promove modulação do SNA, e essa alteração é um aumento do sistema nervoso simpático. A lacuna 3: Ensaio clínico randomizado: Apesar da proximidade do gânglio cervical com $\mathrm{C} 1$ e $\mathrm{C} 2$ vértebras, este estudo mostra que tratamento manipulativo osteopático cervical não produz alteração imediata e 24 horas significativas na PA e FC. 


\section{REFERÊNCIAS}

1. Waitley DD. The autonomic nervous system in osteopathic therapy. October. 1948;47(10):653-6.

2. Fryer G. Intervertebral dysfunction: a discussion of the manipulable spinal lesion. J Osteopath Med [Internet]. 2003 Oct;6(2):64-73. Available from: http://linkinghub.elsevier.com/retrieve/pii/S1443846103800163

3. Ricard F. Tratado De Osteopatia. Madrid: Mandala; 1991. 490 p.

4. Ricard F. Tratamiento Osteopatico T2. madrid; 1993. 600 p.

5. Giles PD, Hensel KL, Pacchia CF, Smith ML. Suboccipital decompression enhances heart rate variability indices of cardiac control in healthy subjects. $J$ Altern Complement Med [Internet]. 2013 Feb [cited 2013 Jul 19];19(2):92-6. Available from: http://www.ncbi.nlm.nih.gov/pubmed/22994907

6. Henley CE, Ivins D, Mills M, Wen FK, Benjamin B a. Osteopathic manipulative treatment and its relationship to autonomic nervous system activity as demonstrated by heart rate variability: a repeated measures study. Osteopath Med Prim Care [Internet]. 2008 Jan [cited 2011 Sep 4];2:7. Available from: http://www.pubmedcentral.nih.gov/articlerender.fcgi?artid=2442110\&tool=pm centrez\&rendertype $=$ abstract

7. Ruffini N, D'Alessandro G, Mariani N, Pollastrelli A, Cardinali L, Cerritelli F. Variations of high frequency parameter of heart rate variability following osteopathic manipulative treatment in healthy subjects compared to control group and sham therapy: randomized controlled trial. Front Neurosci [Internet]. 2015;9(August). Available from:

http://journal.frontiersin.org/Article/10.3389/fnins.2015.00272/abstract

8. Budgell B, Hirano F. Innocuous mechanical stimulation of the neck and alterations in heart-rate variability in healthy young adults. Auton Neurosci [Internet]. 2001 Aug 13;91(1-2):96-9. Available from: http://www.ncbi.nlm.nih.gov/pubmed/11515806

9. Budgell B, Polus B. The effects of thoracic manipulation on heart rate variability: a controlled crossover trial. J Manipulative Physiol Ther [Internet]. 2006 Oct [cited 2013 Aug 29];29(8):603-10. Available from: http://www.ncbi.nlm.nih.gov/pubmed/17045093

10. Roy R a, Boucher JP, Comtois AS. Heart rate variability modulation after manipulation in pain-free patients vs patients in pain. J Manipulative Physiol Ther [Internet]. National University of Health Sciences; 2009 May [cited 2013 Aug 29];32(4):277-86. Available from: http://www.ncbi.nlm.nih.gov/pubmed/19447264 
11. Pickar JG, Bolton PS. Spinal manipulative therapy and somatosensory activation. J Electromyogr Kinesiol [Internet]. Elsevier Ltd; 2012 Oct [cited 2013 Oct 19];22(5):785-94. Available from: http://www.pubmedcentral.nih.gov/articlerender.fcgi?artid=3399029\&tool=pm centrez\&rendertype $=$ abstract

12. Kingston L, Claydon L, Tumilty S. The effects of spinal mobilizations on the sympathetic nervous system: A systematic review. Man Ther [Internet]. Elsevier Ltd; 2014 Apr 13 [cited 2014 May 14]; Available from: http://www.ncbi.nlm.nih.gov/pubmed/24814903

13. Karason AB, Drysdale IP. Somatovisceral response following osteopathic hvlat: a pilot study on the effect of unilateral lumbosacral high-velocity lowamplitude thrust technique on the cutaneous blood flow in the lower limb. J Manipulative Physiol Ther. 2003;26(4):220-5.

14. Lombardini R, Marchesi S, Collebrusco L, Vaudo G, Pasqualini L, Ciuffetti G, et al. The use of osteopathic manipulative treatment as adjuvant therapy in patients with peripheral arterial disease. Man Ther [Internet]. Elsevier Ltd; 2009 Aug [cited 2014 Apr 24];14(4):439-43. Available from: http://www.ncbi.nlm.nih.gov/pubmed/18824395

15. Cerritelli F, Carinci F, Pizzolorusso G, Turi P, Renzetti C, Pizzolorusso F, et al. Osteopathic manipulation as a complementary treatment for the prevention of cardiac complications: 12-Months follow-up of intima media and blood pressure on a cohort affected by hypertension. J Bodyw Mov Ther [Internet]. Elsevier Ltd; 2011 Jan [cited 2013 Jul 19];15(1):68-74. Available from: http://www.ncbi.nlm.nih.gov/pubmed/21147421

16. Mangum K, Partna L, Vavrek D. Spinal Manipulation for the Treatment of Hypertension: A Systematic Qualitative Literature Review. J Manipulative Physiol Ther [Internet]. National University of Health Sciences; 2012;35(3):235-43. Available from: http://linkinghub.elsevier.com/retrieve/pii/S0161475412000267

17. Sutton MSJ, Keane MG. Reverse remodelling in heart failure with cardiac resynchronisation therapy. Heart [Internet]. 2007 Feb [cited 2011 Dec 5];93(2):167-71. Available from: http://www.pubmedcentral.nih.gov/articlerender.fcgi?artid=1861399\&tool=pm centrez\&rendertype $=$ abstract

18. Rogers FJ. Review article The muscle hypothesis : a model of chronic heart failure appropriate. JAOA. 2001;101(10).

19. Olshansky B, Sabbah HN, Hauptman PJ, Colucci WS. Parasympathetic nervous system and heart failure: pathophysiology and potential implications for therapy. Circulation [Internet]. 2008 Aug 19 [cited 2012 Mar 2];118(8):863-71. Available from: http://www.ncbi.nlm.nih.gov/pubmed/18711023 
20. Bibevski S, Dunlap ME. Evidence for impaired vagus nerve activity in heart failure. Heart Fail Rev [Internet]. 2011 Mar [cited 2012 Apr 5];16(2):129-35. Available from: http://www.ncbi.nlm.nih.gov/pubmed/20820912

21. Valen G. Innate immunity and remodelling. Heart Fail Rev [Internet]. 2011 Jan [cited 2011 Oct 7];16(1):71-8. Available from: http://www.pubmedcentral.nih.gov/articlerender.fcgi?artid=3003782\&tool=pm centrez\&rendertype $=$ abstract

22. Marques F, Castro RBP De, Nobre F, Pintya AO, Gallo Jr L, Maciel BC, et al. Replacement of carvedilol for propranolol in patients with heart failure. Arq Bras Cardiol [Internet]. 2010 Jul;95(1):107-14. Available from: http://www.ncbi.nlm.nih.gov/pubmed/20563524

23. Rabito MJ, Kaye AD. Complementary and alternative medicine and cardiovascular disease: an evidence-based review. Evid Based Complement Alternat Med [Internet]. 2013;2013:672097. Available from: http://dx/doi.org/10.1155/2-

13/672097\nhttp://www.pubmedcentral.nih.gov/articlerender.fcgi?artid=36547 $05 \&$ tool $=$ pmcentrez\&rendertype $=$ abstract

24. Ricard F. Tratamiento osteopático de las algias de origen craneo-cervical. madrid: PANAMERICANA; 2001.

25. Ricard F. Tratado De Osteopatia Visceral Y Medicina Interna: Sistema Cardio Respiratorio. [Internet]. $2^{\mathrm{a}}$ edicion. Medos, editor. Madrid, España: Medos; 2015. 384 p. Available from: http://www.amazon.com/Tratado-OsteopatiaVisceral-Medicina-Interna/dp/B00F1HMRKI

26. Corretti MC, Anderson TJ, Benjamin EJ, Celermajer D, Charbonneau F, Creager $\mathrm{M}$ a, et al. Guidelines for the ultrasound assessment of endothelialdependent flow- mediated vasodilation of the brachial artery: a report of the International Brachial Artery Reactivity Task Force. J Am Coll Cardiol. 2002;39(2):257-65.

27. Pyke KE, Tschakovsky ME. The relationship between shear stress and flowmediated dilatation: implications for the assessment of endothelial function. $\mathrm{J}$ Physiol [Internet]. 2005;568(2):357-69. Available from: http://doi.wiley.com/10.1113/jphysiol.2005.089755

28. Niebauer J, Cooke JP. Cardiovascular Effects of Exercise : Role of Endothelial Shear Stress. 1996;28(7):1652-60.

29. Lunt MJ. Review of duplex and colour Doppler imaging of lower-limb arteries and veins. World Wide Wounds. 2000.

30. Wright S a, O'Prey FM, Rea DJ, Plumb RD, Gamble a J, Leahey WJ, et al. Microcirculatory hemodynamics and endothelial dysfunction in systemic lupus erythematosus [Internet]. Arterioscler Thromb Vasc Biol. 2006. p. 2281-7. Available

from: 
http://www.ncbi.nlm.nih.gov/pubmed/16873725\nhttp://atvb.ahajournals.org/c ontent/26/10/2281.full.pdf

31. Logason K, Bärlin T, Jonsson ML, Boström a., Hårdemark HG, Karacagil S. The importance of Doppler angle of insonation on differentiation between 50$69 \%$ and $70-99 \%$ carotid artery stenosis. European Journal of Vascular and Endovascular Surgery. 2001. p. 311-3.

32. Harris R a., Nishiyama SK, Wray DW, Richardson RS. Ultrasound Assessment of Flow-Mediated Dilation. Hypertension. 2010;55(5):1075-85.

33. Chavhan GB, Parra D a, Mann A, Navarro OM. Normal Doppler spectral waveforms of major pediatric vessels: specific patterns. Radiographics. 2008;28(3):691-706.

34. Chuensiri N, Tanaka H, Suksom D. The Acute Effects of Supramaximal HighIntensity Intermittent Exercise on Vascular Function in Lean vs. Pediatr Exerc Sci. 2015;Epub ahead.

35. Thijssen DHJ, Schreuder TH a., Newcomer SW, Laughlin MH, Hopman MTE, Green DJ. Impact of 2-Weeks Continuous Increase in Retrograde Shear Stress on Brachial Artery Vasomotor Function in Young and Older Men. J Am Heart Assoc [Internet]. 2015;4(10):e001968. Available from: http://jaha.ahajournals.org/lookup/doi/10.1161/JAHA.115.001968

36. Atkinson C, Lewis N, Carter H, Thijssen D, Ainslie P, Green D. Impact of sympathetic nervous system activity on post-exercise flow-mediated dilation in humans. J Physiol. 2015;6.

37. Ohsugi K, Sugawara H, Ebina K, Shiga K, Kikuchi N, Mori M, et al. Comparison of brachial artery flow-mediated dilation in youth with type 1 and type 2 diabetes mellitus. J Diabetes Investig [Internet]. 2014;5(5):615-20. Available

from: http://www.pubmedcentral.nih.gov/articlerender.fcgi?artid=4188121\&tool=pm centrez\&rendertype $=$ abstract

38. Peretz A, Leotta DF, Sullivan JH, Trenga C a, Sands FN, Aulet MR, et al. Flow mediated dilation of the brachial artery: an investigation of methods requiring further standardization. BMC Cardiovasc Disord [Internet]. 2007;7:11. Available from: http://www.pubmedcentral.nih.gov/articlerender.fcgi?artid=1847451\&tool=pm centrez\&rendertype $=$ abstract

39. Harris R a, Nishiyama SK, Wray DW, Richardson RS. Ultrasound assessment of flow-mediated dilation. Hypertension [Internet]. 2010 May [cited $2014 \mathrm{Nov}$ 28];55(5):1075-85. Available from: http://www.pubmedcentral.nih.gov/articlerender.fcgi?artid $=2878744 \&$ tool $=$ pm centrez\&rendertype $=$ abstract 
40. Harris R a, Padilla J, Rink LD, Wallace JP. Variability of flow-mediated dilation measurements with repetitive reactive hyperemia. Vasc Med. 2006;11(1):1-6.

41. Hamburg NM, Palmisano J, Larson MG, Sullivan LM, Lehman BT, Vasan RS, et al. Relation of brachial and digital measures of vascular function in the community: the Framingham heart study. Hypertension [Internet]. 2011;57(3):390-6. Available from: http://www.pubmedcentral.nih.gov/articlerender.fcgi?artid=3049726\&tool=pm centrez\&rendertype $=$ abstract

42. Shechter M, Shalmon G, Scheinowitz M, Koren-Morag N, Feinberg MS, Harats D, et al. Impact of acute caffeine ingestion on endothelial function in subjects with and without coronary artery disease. Am J Cardiol [Internet]. 2011;107(9):1255-61. Available from: http://www.ncbi.nlm.nih.gov/pubmed/21349479

43. Buscemi S, Verga S, Batsis J a, Donatelli M, Tranchina MR, Belmonte S, et al. Acute effects of coffee on endothelial function in healthy subjects. Eur J Clin Nutr [Internet]. BioMed Central Ltd; 2010;64(5):483-9. Available from: http://eds.b.ebscohost.com.ezproxy.endeavour.edu.au/eds/pdfviewer/pdfviewer ?vid=11\&sid=f370d84a-d935-46e7-8f4a-

2bcaace88506@sessionmgr115\&hid=113

44. Rakobowchuk M, Parsloe ER, Gibbins SE, Harris E, Birch KM. Prolonged low flow reduces reactive hyperemia and augments low flow mediated constriction in the brachial artery independent of the menstrual cycle. PLoS One [Internet]. 2013;8(2):e55385. Available from: http://www.pubmedcentral.nih.gov/articlerender.fcgi?artid=3564810\&tool=pm centrez\&rendertype $=$ abstract

45. Thijssen DHJ, Black M a., Pyke KE, Padilla J, Atkinson G, Harris R a., et al. Assessment of flow-mediated dilation in humans: a methodological and physiological guideline. AJP Hear Circ Physiol [Internet]. 2011;300(1):H2-12. Available from: http://ajpheart.physiology.org/cgi/doi/10.1152/ajpheart.00471.2010

46. Ercan E, K?r?lmaz B, Kahraman ?Smail, Bayram V, Do?an H. New fully automated software for assessment of brachial artery flow- mediated dilation with advantages of continuous measurement. Anadolu Kardiyol Derg [Internet]. 2012;12(7):553-9. Available from: http://www.ncbi.nlm.nih.gov/pubmed/22877899

47. Kizhakekuttu TJ, Gutterman DD, Phillips S a, Jurva JW, Arthur EIL, Das E, et al. Measuring FMD in the brachial artery: how important is QRS gating? J Appl Physiol [Internet]. 2010;109(4):959-65. Available from: http://www.pubmedcentral.nih.gov/articlerender.fcgi?artid=2963331\&tool=pm centrez\&rendertype $=$ abstract 
48. Alley H, Owens CD, Gasper WJ, Grenon SM. Ultrasound assessment of endothelial-dependent flow-mediated vasodilation of the brachial artery in clinical research. Journal of visualized experiments : JoVE. 2014. p. e52070.

49. Donnelly R, Hinwood D, London NJ. ABC of arterial and venous disease. Non-invasive methods of arterial and venous assessment. BMJ. 2000;320(7236):698-701.

50. Gerhard-Herman M, Gardin JM, Jaff M, Mohler E, Roman M, Naqvi TZ. Guidelines for Noninvasive Vascular Laboratory Testing: A Report from the American Society of Echocardiography and the Society of Vascular Medicine and Biology. J Am Soc Echocardiogr. 2006;19(8):955-72.

51. Quiñones $\mathrm{M}$ a, Otto CM, Stoddard M, Waggoner A, Zoghbi W a. Recommendations for quantification of Doppler echocardiography: a report from the Doppler Quantification Task Force of the Nomenclature and Standards Committee of the American Society of Echocardiography. J Am Soc Echocardiogr. 2002;15(2):167-84.

52. Zilla P, Moodley L, Scherman J, Krynauw H, Kortsmit J, Human P, et al. Remodeling leads to distinctly more intimal hyperplasia in coronary than in infrainguinal vein grafts. J Vasc Surg [Internet]. Elsevier Inc.; 2012;55(6):1734-41. Available from: http://dx.doi.org/10.1016/j.jvs.2011.11.057

53. Holland CK, Brown JM, Scoutt LM, Taylor KJ. Lower extremity volumetric arterial blood flow in normal subjects. Ultrasound Med Biol. 1998;24(8):107986.

54. Nilsson S. Comparative anatomy of the autonomic nervous system. Auton Neurosci [Internet]. Elsevier B.V.; 2011 Nov 16 [cited 2013 Sep 3];165(1):3-9. Available from: http://www.ncbi.nlm.nih.gov/pubmed/20444653

55. Netter F. Atlas de anatomia humana. 5 ed. São Paulo: Elsevier; 2011. 1090 p.

56. Moore K, Dalley A. Anatomia Orientada para a Prática Clínica. 5 ed. Rio de Janeiro: Guanabara Koogan; 2007.

57. Machado A. Neuroanatomia Funcional. São Paulo: Atheneu; 1991.

58. Thibodeau GA., Patton KT. Estrutura e Funções do Corpo Humano. 9 ed. Rio de Janeiro: Guanabara Koogan; 2002.

59. Guyton A, Hall. Fisiologia humana. Rio de Janeiro: Elsevier; 2011.

60. Bellary SS, Walters A, Gielecki J, Shoja MM, Tubbs RS, Loukas M. Jacob B. Winslow (1669-1760). Clin Anat [Internet]. 2012;25(5):545-7. Available from: http://doi.wiley.com/10.1002/ca.22033 
61. Furness JB. The organisation of the autonomic nervous system: peripheral connections. Auton Neurosci [Internet]. 2006 Dec 30 [cited 2013 Sep 3];130(12):1-5. Available from: http://www.ncbi.nlm.nih.gov/pubmed/16798102

62. Gardner, Gray. Anatomia. Rio de Janeiro: : Guanabara Koogan; 1988.

63. Hashimoto K, Kuré K, Okinaka S. Spinal parasympatethic fibres in the cervical sympathetic. Exp Physiol. 1934;XXIV(3).

64. Zaręba W. Comparison of clinical trials evaluating cardiac resynchronization therapy in mild to moderate heart failure. Cardiol J [Internet]. 2010 Jan;17(6):543-8. Available from: http://www.ncbi.nlm.nih.gov/pubmed/21154255

65. Zucker IH, Patel KP, Schultz HD. Neurohumoral stimulation. Heart Fail Clin [Internet]. Elsevier Inc; 2012 Jan [cited 2012 Nov 28];8(1):87-99. Available from: http://www.ncbi.nlm.nih.gov/pubmed/22108729

66. Keller DM, Cui J, Davis SL, Low D a, Crandall CG. Heat stress enhances arterial baroreflex control of muscle sympathetic nerve activity via increased sensitivity of burst gating, not burst area, in humans. J Physiol [Internet]. 2006 Jun 1 [cited 2012 Apr 6];573(Pt 2):445-51. Available from: http://www.pubmedcentral.nih.gov/articlerender.fcgi?artid=1779723\&tool=pm centrez\&rendertype $=$ abstract

67. Feng B, Li B, Nauman E a, Schild JH. Theoretical and electrophysiological evidence for axial loading about aortic baroreceptor nerve terminals in rats. Am J Physiol Heart Circ Physiol [Internet]. 2007 Dec [cited 2012 Apr 6];293(6):H3659-72. Available from: http://www.ncbi.nlm.nih.gov/pubmed/17951369

68. Guyton AC, Estañol B, Porras-betancourt M, Padilla-leyva MÁ. Breve historia del reflejo barorreceptor: de Claude Bernard a. Arch Cardiol Mex. 2011;81(4):330-6.

69. Fisher JP, Young CN, Fadel PJ. Effect of muscle metaboreflex activation on carotid-cardiac baroreflex function in humans. Am J Physiol Heart Circ Physiol [Internet]. 2008 May [cited 2012 Apr 6];294(5):H2296-304. Available from: http://www.ncbi.nlm.nih.gov/pubmed/18326794

70. Fisher JP, Seifert T, Hartwich D, Young CN, Secher NH, Fadel PJ. Autonomic control of heart rate by metabolically sensitive skeletal muscle afferents in humans. J Physiol [Internet]. 2010 Apr 1 [cited 2012 Mar 19];588(Pt 7):111727. Available from: http://www.pubmedcentral.nih.gov/articlerender.fcgi?artid=2852999\&tool=pm centrez\&rendertype $=$ abstract

71. Verdecchia P, Angeli F, Cavallini C, Gattobigio R, Gentile G, Staessen J a, et al. Blood pressure reduction and renin-angiotensin system inhibition for prevention of congestive heart failure: a meta-analysis. Eur Heart J [Internet]. 
2009 Mar [cited 2012 Mar 5];30(6):679-88. Available from: http://www.ncbi.nlm.nih.gov/pubmed/19168534

72. Grassi G, Mancia G. New therapeutic approaches for resistant hypertension. J Nephrol [Internet]. 2012 [cited 2012 Nov 3];25(3):276-81. Available from: http://www.ncbi.nlm.nih.gov/pubmed/22362551

73. Irigoyen MC, Consolim-colombo FM, Krieger EM. Controle cardiovascular : regulação reflexa e papel do sistema nervoso simpático. Rev Bras Hipertens. 2001;8(1):55-62.

74. Vieira PJC, Ribeiro JP, Cipriano G, Umpierre D, Cahalin LP, Moraes RS, et al. Effect of transcutaneous electrical nerve stimulation on muscle metaboreflex in healthy young and older subjects. Eur J Appl Physiol. 2011;

75. Thayer JF, Yamamoto SS, Brosschot JF. The relationship of autonomic imbalance, heart rate variability and cardiovascular disease risk factors. Int $\mathbf{J}$ Cardiol [Internet]. Elsevier Ireland Ltd; 2010 May 28 [cited 2013 May 22];141(2):122-31. Available from: http://www.ncbi.nlm.nih.gov/pubmed/19910061

76. Angelis K De, Santos M do SB, Irigoyen MC. Sistema nervoso autônomo e doença cardiovascular. Rev da Soc Cardiol do Rio Gd do Sul. 2004;(Figura 1):1-7.

77. Thayer JF, Lane RD. The role of vagal function in the risk for cardiovascular disease and mortality. Biol Psychol [Internet]. 2007 Feb [cited 2013 Sep 23];74(2):224-42. Available

from: http://www.ncbi.nlm.nih.gov/pubmed/17182165

78. Bootsma M, Swenne CA, Janssen MJA, Cats VM, Schalij MJ. Heart rate variability and sympathovagal balance: pharmacological validation. Netherlands Hear Journal,. 2003;11(6).

79. Marín J, Marín E, Gutiérrez-Iñiguez M a, Avendaño C, Rodríguez-Martínez M a. Mechanisms involved in the hemodynamic alterations in congestive heart failure as a basis for a rational pharmacological treatment. Pharmacol Ther [Internet]. $2000 \quad$ Oct;88(1):15-31. Available from: http://www.ncbi.nlm.nih.gov/pubmed/11033382

80. Zhang GQ, Zhang W. Heart rate, lifespan, and mortality risk. Ageing Res Rev [Internet]. 2009 Jan [cited 2013 Oct 5];8(1):52-60. Available from: http://www.ncbi.nlm.nih.gov/pubmed/19022405

81. Grant CC, Viljoen M, Janse van Rensburg DC, Wood PS. Heart rate variability assessment of the effect of physical training on autonomic cardiac control. Ann Noninvasive Electrocardiol [Internet]. 2012 Jul [cited 2013 Aug 31];17(3):219-29. Available from: http://www.ncbi.nlm.nih.gov/pubmed/22816541 
82. Cole C, Blackstone E, Pashkow F, Snader C, Lauer M. Heart-rate recovery immediately after exercise as a predictor of mortality. $\mathrm{N}$ Engl J Med. 1999;341(18):1351-7.

83. Radespiel-tröger M, Gottschalk T, Mück-weymann M. Agreement of two different methods for measurement of heart rate variability. Clin Aut Res. 2003;99-102.

84. Kingsley M, Lewis MJ, Marson RE. Comparison of Polar 810s and an ambulatory ECG system for RR interval measurement during progressive exercise. International Journal of Sports Medicine. 2005. p. 39-44.

85. Berthoin S, Bosquet L. Validity of the Polar S810 Heart Rate Monitor to Measure R-R Intervals at Rest. Med Sci Sport Exerc. 2006;887-93.

86. Wallén MB, Hasson D, Theorell T, Canlon B, Osika W. Possibilities and limitations of the Polar RS800 in measuring heart rate variability at rest. Eur J Appl Physiol [Internet]. 2012 Mar [cited 2013 Sep 24];112(3):1153-65. Available from: http://www.ncbi.nlm.nih.gov/pubmed/21766225

87. Force T, Society E, North T, Society A. Guidelines Heart rate variability. Eur Heart J. 1996;17:354-81.

88. Smith A-L, Owen H, Reynolds KJ. Heart rate variability indices for very shortterm (30 beat) analysis. Part 1: survey and toolbox. J Clin Monit Comput [Internet]. 2013 Oct [cited 2013 Oct 19];27(5):569-76. Available from: http://www.ncbi.nlm.nih.gov/pubmed/23674071

89. Tannus LRM, Sperandei S, Montenegro Júnior RM, Carvalho VR, Pedrosa HC, Félix MT, et al. Reproducibility of methods used for the assessment of autonomous nervous system's function. Auton Neurosci [Internet]. Elsevier B.V.; 2013 Oct [cited 2013 Oct 19];177(2):275-9. Available from: http://www.ncbi.nlm.nih.gov/pubmed/23770193

90. Lauer MS. Autonomic function and prognosis. Cleve Clin J Med [Internet]. 2009 Apr [cited 2013 Jun 6];76 Suppl 2:S18-22. Available from: http://www.ncbi.nlm.nih.gov/pubmed/19376976

91. Neves VR, Takahashi ACM, do Santos-Hiss MDB, Kiviniemi AM, Tulppo MP, de Moura SCG, et al. Linear and nonlinear analysis of heart rate variability in coronary disease. Clin Auton Res [Internet]. 2012 Aug [cited 2013 Oct 1];22(4):175-83. Available from: http://www.ncbi.nlm.nih.gov/pubmed/22476435

92. Vanderlei LCM, Patre CM, Hoshi RA, Carvalo TD de, Godoy MF de. Noções básicas de variabilidade da frequência cardíaca e sua aplicabilidade clínica. Rev Bras Cir Cardiovasc 2009; 2009;24(2):205-17.

93. Archiza B, Simões RP, Mendes RG, Fregonezi G a F, Catai AM, Borghi-Silva A. Acute effects of different inspiratory resistive loading on heart rate 
variability in healthy elderly patients. Brazilian J Phys Ther [Internet]. 2013;17(4):401-8. Available

from:

http://www.ncbi.nlm.nih.gov/pubmed/23970114

94. Smith A-L, Owen H, Reynolds KJ. Heart rate variability indices for very shortterm (30 beat) analysis. Part 2: validation. J Clin Monit Comput [Internet]. 2013 Oct [cited 2013 Oct 19];27(5):577-85. Available from: http://www.ncbi.nlm.nih.gov/pubmed/23681923

ANEXO 1 - PRODUÇÕES DURANTE O DOUTORADO

RESUMOS EXPANDIDOS PUBLICADOS EM ANAIS DE CONGRESSOS 1. 
AMATUZZI TEIXEIRA; QUINTANILHA, H. L. R. M. ; BRANCO, A. C. ; THOMAZ, S. R.; NAKATA, C. H. ; SILVA, M. ; CIPRIANO JUNIOR, G. . A manipulação Osteopática cevical gera efeitos imediatos na variáveis cardiovasculares em indivíduos saudáveis?. In: congresso da SOCESP, 2015, SÃO PAULO. cardiologia interdisciplinar integrando o humano pelo coração. sao paulo: farol editora, 2015. v. 25. p. 227.

2.

SILVA, V. M. ; LIMA, A. ; AMATUZZI TEIXEIRA ; NEVES, L. ; SILVA, M. ; BOTTARO, M. ; ARENA, R. ; GURNEY, B. ; CIPRIANO JUNIOR, G. ; CIPRIANO, G. . Non-Invasive Ventilation Improves Cardiovascular Adjustments And Fatigability In Patients With Heart Failure. 2013. ATS International Conference, Philadelphia.

\section{APRESENTAÇÕES DE TRABALHO}

1.

AMATUZZI TEIXEIRA, F. ; QUINTANILHA, H. L. ; DUARTE, A. C. B. ; THOMAZ, S. ; NAKATA, C. H. ; SILVA, M. L. ; CIPRIANO JUNIOR, G. . A MANIPULAÇÃO OSTEOPÁTICA CERVICAL GERA EFEITOS IMEDIATOS NAS VARIÁVEIS CARDIOVASCULARES EM INDIVÍDUOS SAUDÁVEIS? 2015. (Apresentação de Trabalho/Congresso). Congresso da SOCESP, São Paulo.

2.

AMATUZZI TEIXEIRA, F. ; XAVIER, A. P. ; GOMES, E. ; NAKATA, C. H. ; THOMAZ, S. ; SILVA, M. L. ; CIPRIANO JUNIOR, G. . EFEITOS AGUDOS DO TRATAMENTO MANIPULATIVO OSTEOPÁTICO NA FUNÇÃO AUTONÔMICA EM IDOSOS. 2015. (Apresentação de Trabalho/Congresso). Congresso da SOCESP, São Paulo.

3.

NAKATA, C. H. ; AMATUZZI TEIXEIRA, F. ; Urache LVT ; SILVA, M. L. ; THOMAZ, S. ; LIMA, A. C. G. B. ; SANTOS, F. V. ; BORGES, R. F. ; CIPRIANO JUNIOR, G. . ACUTE EFFECTS OF INTERFERENTIAL ELECTRICAL STIMULATION ON HEART RATE VARIABILITY IN HEALTHY WOMEN. 2015. (Apresentação de Trabalho/Congresso). AACVPR 30th Annual Meeting, Washington. 
4.

AMATUZZI TEIXEIRA; CIPRIANO JUNIOR, G. . 1 workshop de redação de artigos científicos. 2015. (Apresentação de Trabalho/Outra). Universidade de Brasília $-\mathrm{UnB}$.

5.

AMATUZZI TEIXEIRA, QUEIROZ, BARREIRA, LISSA, OLIVEIRA, CASTELO BRANCO, MALDANER, JÁCOMO, CIPRIANO JÚNIOR. ACUTE EFFECTS OF OSTEOPATHIC MANIPULATIVE TREATMENT IN HEART RATE VARIABILITY IN PATIENTS WITH HEART FAILURE: A CROSS-OVER STUDY. 2014. (Congresso). Heart Failure Congress, Athenas.

6. SILVA, V. M. ; LIMA, A. ; NEVES, L. ; AMATUZZI TEIXEIRA ; SILVA, M. ; BOTTARO, M. ; ARENA, R. ; GURNEY, B. ; CIPRIANO JUNIOR, G. ; CIPRIANO, G. . Non-Invasive Ventilation Improves Cardiovascular Adjustments And Fatigability In Patients With Heart Failure. 2013. (Apresentação de Trabalho/Congresso). ATS International Conference, Philadelphia

7.

AMATUZZI TEIXEIRA ; Osteopatia e Sistema Nervoso Autonômico: Novas Perspectivas. 2013. (Apresentação de Trabalho/Congresso).CIOST - Congresso internacional de Osteopatia, Foz do Iguaçu.

\section{INICIAÇÃO CIENTÍFICA}

1.

ISABELLE BARREIRA. INFLUÊNCIA AGUDA DO TRATAMENTO MANIPULATIVO OSTEOPÁTICO (TMO) NO SISTEMA NERVOSO AUTONOMICO (SNA) EM INDIVÍDUOS JOVENS. 2013. Iniciação Científica. (Graduando em Fisioterapia) -Universidade de Brasília. Orientador: Fellipe Amatuzzi Teixeira.

2.

EMILLY KAROLINE DE SOUZA GOMES. EFEITOS DO TRATAMENTO MANIPULATIVO OSTEOPÁTICO NA PRIMEIRA COSTELA NA PRESSÃO ARTERIAL DE JOVENS SAUDÁVEIS. Início: 2014. Iniciação científica (Graduando em Fisioterapia) - Universidade de Brasília, Conselho Nacional de 
Desenvolvimento Científico e Tecnológico. (Orientador).

\section{TRABALHO DE CONCLUSÃO DE CURSO DE GRADUAÇÃO}

1.

HANNA LISSA RIBEIRO MIRANDA QUINTANILHA E AMANDA CASTELO BRANCO DUARTE. EFEITOS IMEDIATOS DA MANIPULAÇÃO OSTEOPÁTICA CERVICAL NAS VARIÁVEIS CARDIOVASCULARES EM INDIVÍDUOS SAUDÁVEIS. 2014. Trabalho de Conclusão de Curso. (Graduação em Fisioterapia) - Universidade de Brasília. Orientador: Fellipe Amatuzzi Teixeira.

2.

ANA PAULA PEREIRA XAVIER. EFEITOS IMEDIATOS DA MANIPULAÇÃO OSTEOPÁTICA CERVICAL NAS VARIÁVEIS CARDIOVASCULARES EM INDIVÍDUOS IDOSOS. 2014. Trabalho de Conclusão de Curso. (Graduação em Fisioterapia) - Universidade de Brasília. Orientador: Fellipe Amatuzzi Teixeira.

3.

LUCAS CERATTI SILVELLO DE MELLO LIMA. EFEITO DO TRATAMENTO MANIPULATIVO OSTEOPÁTICO SOBRE O FLUXO SANGUÍNEO E A PRESSÃO ARTERIAL EM INDVÍDUOS SAUDÁVEIS. 2015. Trabalho de Conclusão de Curso (Graduação em Fisioterapia) - Universidade de Brasília. Orientador: Sérgio Ricardo Thomaz, Co-orientador: Fellipe Amatuzzi Teixeira.ANEXO 2 - PARECER DO COMITÊ DE ÉTICA E PESQUISA. 


FACULDADE DE CIÊNCIAS DA
SAUUDE DA UNIVERSIDADE DE
BnB Plotoformo
Bralili - CEP/FS-UNB

\section{PARECER CONSUBSTANCIADO DO CEP}

DADOS DO PROJETO DE PESQUISA

Titulo da Pesquisa: EFEITOS DO TRATAMENTO MANIPULATIVO OSTEOPATICO (TMO) NA FUNCIONALIDADE DE PACIENTES COM INSUFICIENCIA CARDIACA CONGESTIVA: UM ENSAIO CLINICO RANDOMIZADO

Pesquisador: FELLIPE AMATUZZI TEIXEIRA

Area Tematica:

Versalo: 2

CAAE: 10146913.2 .0000 .0030

Institulçao Proponente:PROGRAMA DE POS-GRADUAÇÅO EM CIÉNCIAS E TECNOLOGIAS EM

Patrocinador Princlpal: FInanclamento Proprio

DADOS DO PARECER

Nümero do Parecer: 378.381

Data da Relatorla: 24/07/2013

Apresentaçåo do Projeto:

VIde parecer anterlor

Objetivo da Pesqulsa:

Vide parecer anterlor

Avallaça dos Rlacos $\theta$ Beneficlos:

VIde parecer anterior

Comentarlos e Consideraçoes sobre a Pesqulsa:

VIde parecer anterlor

Consideraç0өs sobre 08 Termos de apresentaça obrigatorla:

Vlde parecer anterior

Recomendaç0өs:

Vide parecer anterior

Conclusoes ou Pendenclas e Llsta de Inadequaçoes:

As pendenclas sobre o TCLE foram atendidas. Ressalva-se a Importancla de adiclonar o logo da UnB no mesmo.

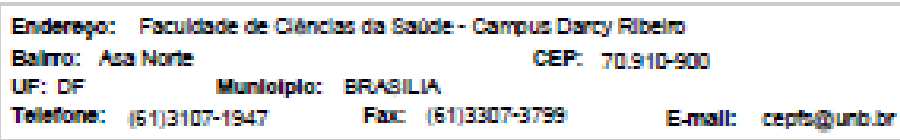




FACULDADE DE CIÊNCIAS DA
UnB
SAUUDE DA UNIVERSIDADE DE
BRASILIA - CEPIFS-UNB

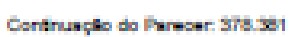

situaçà do Parecer:

Aprovado

Necessita Apreclaçăa da CONEP:

N5o

Consideraçôes Finals a criterlo do CEP:

BRASILIA, 30 de Agosto de 2013

Assinador por:

Natan Monsores de Sa

(Coordenador)

Enderego: Faculadade de Clenclas da Saude-Campue Darcy Fibeiro

Boirro: Aea Norte CEP: 70.910-900

Telatone: [51]3107-1947 Fax (61]3307-3799 E-malt: ceptagurbibr

Paina $\infty$ on os 
ANEXO 3 - REGISTRO BRASILEIRO DE ENSAIOS CLÍNICOS

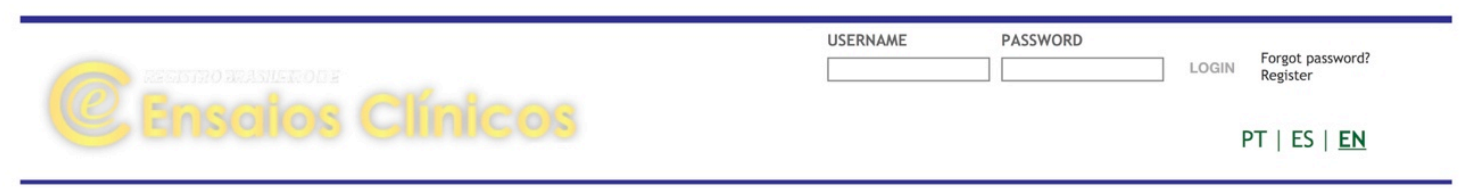

NEWS | ABOUT | HELP | CONTACT

ADVANCED SEARCH

Search trials

HOME / REGLSTERED TRIALS /

RBR-3st9f3

Effects of osteopathic manipulative treatment ( OMT )in functionality of patients with congestive heart failure: a randomized clinical trial .

Registration Date: March 3, 2015, 3:07 p.m.

Last Update: April 17, 2015, 2:23 p.m.

Study Type:

Intervention Study

Scientific Title:

Efeitos do Tratamento manipulativo
osteopático (TMO) na Funcionalidade de
pacientes com insuficiência cardiaca
congestiva: um ensaio clínico randomizado.

Effects of osteopathic manipulative EN treatment ( OMT ) in functionality of patients with congestive heart failure: a randomized clinical trial .

Trial Identification

UTN Number: U1111-1167-8725

Public Title:

Pfeito da Osteopatia nos pacientes com
doença cardiaca. $\begin{aligned} & \text { Osteopathic effects in patientes with heart } \\ & \text { disease }\end{aligned}$

Scientific Acronym:

Public Acronym

Secondary Identifying Numbers:

378.381/2013

Issuing Authority: comitê de ética FS/UnB

CAAE: 10146913.2.0000.0030

Issuing Authority: Sistema Nacional de Ética em Pesquisa - SISNEP

Sponsors

Primary Sponsor: FCE - Faculdade de Ceilândia

Secondary Sponsors: 
Institution: FCE - Faculdade de Ceilândia

Source(s) of Monetary or Material Support:

Institution: FCE - Faculdade de Ceilândia

\title{
Health Conditions
}

Health Condition(s) or Problem(s):

doença cardiovascular, insuficiência
cardiaca congestiva, doença arterial
periférica, rastreamento do sistema
nervoso autonômico em jovens e em
pessoas idosas.

congestive heart failure, peripheral artery disease, tracking the autonomic nervous system in young and elderly.

General Descriptors for Health Condition(s):
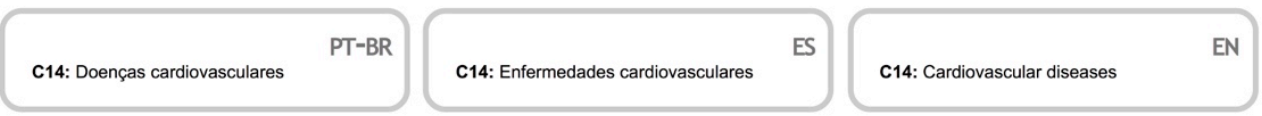

Specific Descriptors for Health Condition(s):

151.6: Doença cardiovascular não
especificada

151.6: Enfermedad cardiovascular, no especificada

ES
EN

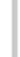

PT-BR

125.0: Doença cardiovascular aterosclerótica, descrita desta maneira

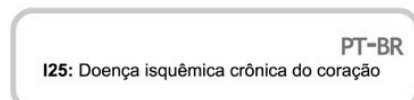

PT-BR

G09.330.190: Processos Fisiológicos Cardiovasculares

\section{0: Enfermedad cardiovascular aterosclerótica, asi descrita \\ ES}

125: Enfermedad isquémica crónica del corazón
ES
125.0: Atherosclerotic cardiovascular disease, so described

125: Chronic ischaemic heart disease

EN

ES

G09.330.190: Procesos Fisiológicos Cardiovasculares
EN

G09.330.190: Cardiovascular Physiological Processes

Interventions

\begin{abstract}
Intervention Code(s)
Dietary supplement

Other
\end{abstract}

Interventions: 


PT-BR
Grupo experimental (GE): 30 pacientes
cardiopatas serão tratados por meio de
tratamento manipulativo osteopático que
consiste em manipulações da coluna
vertebral, tecidos moles, visceras e fáscias
por 2 meses, 1 vez por semana. Grupo
sham (GS): Os mesmos 30 pacientes
cardiopatas que receberão tratamento
também participarão do grupo sham que
será feito por meio de simulação do
tratamento osteopático por meio de toque
na pele leve sem intenção de tratamento
por mais 2 meses, 1 vez por semana.
Grupo Controle (GC): 30 indivíduos sem
doença cardiaca não receberão nenhum
tipo de intervenção de tratamento, fará
apenas as avaliaçães iniciais e finais.

Experimental group (EG): 30 cardiac patients will be treated by manipulative osteopathic treatment consisting of spinal manipulations , soft tissue , fascia and viscera for 2 months, 1 time per week. Sham group (SG): The same 30 patients with heart disease who receive treatment also participate in the sham group that will be done by simulation of osteopathic treatment through touch the light skin unintentionally treatment for over 2 months , 1 time per week. Control Group (CG ): 30 patients without heart disease will not receive any treatment intervention, will only the initial and final evaluations .

Descriptor for Intervention(s):

E02.190.599.280: Manipulação
Osteopática

E02.190.599.280: Manipulación Osteopática

\begin{tabular}{l} 
E02.190.599: Manipulaçōes \\
Musculoesqueléticas \\
\hline \\
E02.190.599.233: Manipulação \\
Quiroprática
\end{tabular}

\begin{tabular}{l} 
E02.190.599: Manipulaciones \\
Musculoesqueléticas \\
\hline \\
E02.190.599.233: Manipulación \\
Quiropráctica
\end{tabular}

\section{Recruitment}

Recruitment Status: Recruiting

Recruitment Country

Brazil

Planned Date of First Enrollment: 2015-04-06

Planned Date of Last Enrollment: 2015-10-06

\begin{tabular}{llll} 
Target Sample Size: & Gender (inclusion sex): & Inclusion Minimum Age: & Inclusion Maximum Age: \\
\hline 50 & $-18 \mathrm{Y}$ & $90 \mathrm{Y}$ \\
\hline
\end{tabular}

Inclusion Criteria:

Para o grupo experimental: Insuficiência


cardiaca congestiva, doença cardiovascular, doença de chagas Para o grupo controle: não ter doença cardiovascular e ser maior de 18 anos.

heart failure , cardiovascular disease,

Chagas disease

For the control group : not having

cardiovascular disease and be over 18 years.

\section{Exclusion Criteria:}

PT-BR
Para o grupo experimental: New York Heart
Association classe I, paciente
descompensado hemodinamicamente,
sinais ou sintomas de piora cardiovascular
nos últimos 3 meses.
Para o grupo controle: Doença
cardiovascular, hipertensão, cirurgias,
fibromioalgia, disautonomia.

For the experimental group : New York

EN Heart Association class I, hemodynamically decompensated patient, signs or symptoms of cardiovascular worsening in the last 3 months.

For the control group : cardiovascular disease, hypertension, surgeries , fibromioalgia , dysautonomia.

\section{Study Type}

Study Design:

PT-BR
Ensaio clinico de tratamento, randomizado,
controlado, cruzado, duplo-cego, com três
braços

Clinical trial of treatment, randomized, controlled, crossover , double -blind, threearm

\begin{tabular}{lll|l|l|l|l} 
Expanded access program & Study Purpose & Intervention Assignment & Number of arms & Masking type & Allocation type & Study Phase \\
\hline False & Treatment & Cross-over & 3 & Double-blind & Randomized-controlled & N/A \\
\hline
\end{tabular}

\section{Outcomes}

Primary Outcomes:

\begin{tabular}{l|l|}
$\begin{array}{l}\text { Melhora da capacidade fisica do } \\
\text { cardiopata, mediante o teste de esforço } \\
\text { cardiopulmonar com pelo menos } 20 \% \text { do } \\
\text { aumento dos valores iniciais no grupo } \\
\text { experimental. }\end{array}$ & $\begin{array}{l}\text { EN } \\
\text { improvement in physical function in cardiac } \\
\text { by cardiopulmonary exercise test with at } \\
\text { least } 20 \% \text { of the increase from baseline in } \\
\text { the experimental group. }\end{array}$ \\
\end{tabular}

Secondary Outcomes:

nento da variabilidade da frequenc cardiaca, verificadas pela variabilidade da frequência cardiaca em pelo menos $20 \%$ do valor inicial no grupo experimental; aumento do fluxo sanguíneo na artéria braquial, verificado por meio da avaliação 
de fluxo mediado por vasodilatação mensurado em percentual, com pelo menos $20 \%$ de aumento do valor inicial no grupo experimental; diminuição dos marcadores bioquímicos de sangue, verificados por meio de exame sanguineo de pelo menos $20 \%$ de diminuição do valor inicial no grupo experimental: at least $20 \%$ of its initial value increased in the experimental group

decrease of blood biochemical markers, the blood verified by examining at least $20 \%$ decrease from baseline in experimental group ;

Contacts

Contacts for Public Queries

Full Name: Fellipe Amatuzzi Teixeira

Address: Centro Metropolitano, conjunto A, lote

01, Brasília - DF.

City: Brasilia / Brazi

Zip Code: $72220-900$

Telephone: +556199667264

E-mail: famatuzzi@gmail.com

Affiliation: Universidade de Brasilia

\section{Contacts for Scientific Queries}

Full Name: Fellipe Amatuzzi Teixeira

Address: Centro Metropolitano, conjunto A, lote

01, Brasília - DF.

City: Brasilia / Brazi

Zip Code: $72220-900$

Telephone: +556199667264

E-mail: famatuzzi@gmail.com

Affiliation: Universidade de Brasília

Contact(s) for Site Queries

Full Name: Fellipe Amatuzzi Teixeira

Address: Centro Metropolitano, conjunto A, lote 01, Brasília - DF.

City: Brasilia / Brazil

Zip Code: $72220-900$

Telephone: +556199667264

E-mail: famatuzzi@gmail.com

Affiliation: Universidade de Brasília 
Additional Links:

Download as ICTRP format

Download as OpenTrials XML format

$\checkmark$ OpenTrials v1.2 
ANEXO 4 - NORMAS DA REVISTA E QUALIS - MANUSCRITO 1.

\section{JOURNAL OF PHYSIOTHERAPY - FATOR DE IMPACTO 3,1}
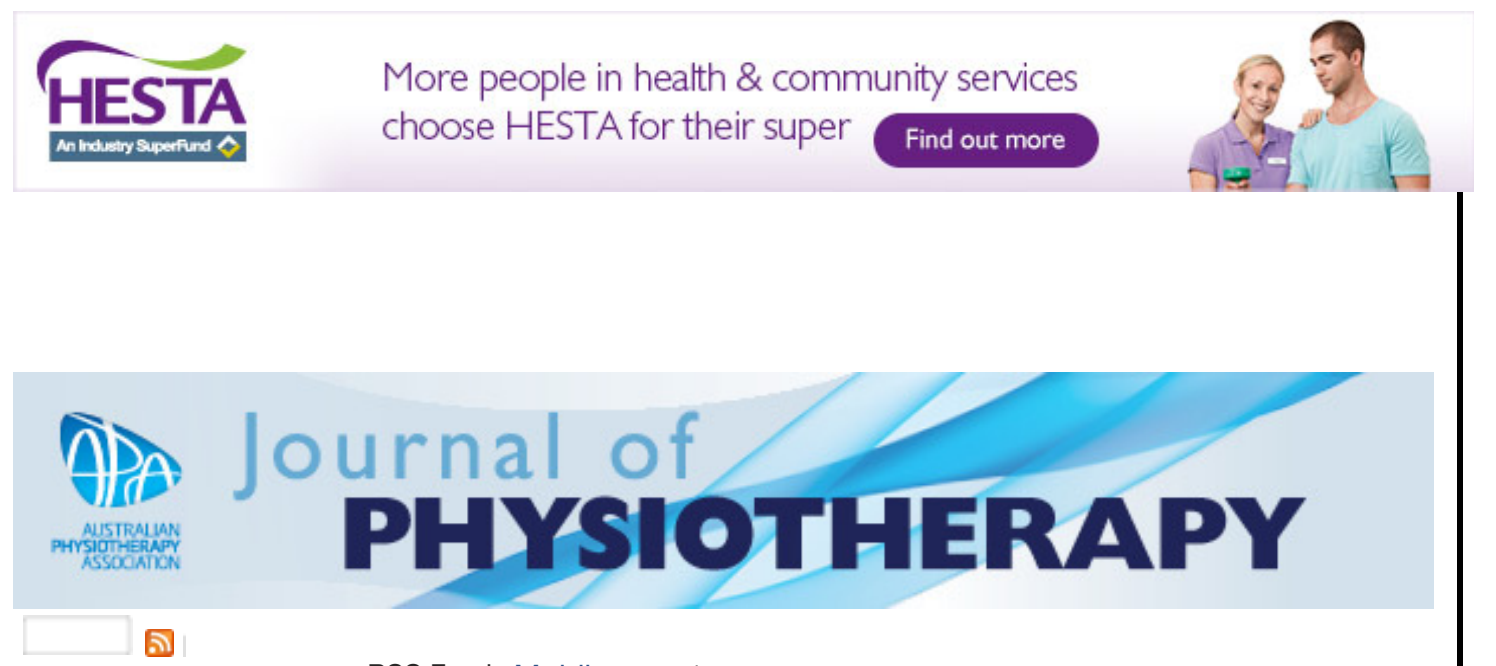

APA member access information RSS Feeds Mobile Login | Register

Articles \& Issues For Authors APA Research Journal Info Order Print Copy More Periodicals Manual Therapy journal

All Content Search Advanced SearchJournal of Physiotherapy (JoP) is the first Open Access core physiotherapy journal; it welcomes contributions that are relevant to the science or practice of physiotherapy.

\section{CONTENT Original Research}

The Editorial Board is committed to publishing excellent research and will consider the following types of papers:

- Systematic reviewsSystematic reviews are strongly preferred over narrative (non-systematic) reviews. High quality systematic reviews with firm conclusions are a publication priority. However, systematic reviews are unlikely to be published if they find there is not enough good quality evidence to review or if the literature is inconclusive. Note that this journal gives priority to systematic reviews that are prospectively registered in a publicly available register (e.g., PROSPERO at http://www.crd.york.ac.uk/PROSPERO). Authors should submit evidence of registration when submitting a manuscript for consideration. There are specific guidelines available for this type of study at the end of the Presentation section of these Author Guidelines. C Clinical trialsAll clinical trials submitted to JoP must have been registered in a publicly-accessible trials register. We will accept any register that satisfies the International Committee of Medical Journal Editors requirements (such as The Australian New Zealand Clinical Trial Registry at http://www.anzctr.org.au). Authors must provide the name and website address of the register and the trial registration number on submission. The journal will only accept trials that have been registered prospectively unless data collection began before 2006, in which case retrospective registration is acceptable. There are specific guidelines available for this type of study at the end of the Presentation section. Economic analyses• Experimental studies• Qualitative studiesQualitative research refers to research where the analysis of data involves qualitative judgements. Commonly qualitative research explores aspects of the human, social world. Qualitative research methodologies include narrative inquiry, case studies, naturalistic inquiry, ethnography, hermeneutics, phenomenology, and survey research using open-ended questions. There are specific guidelines available for this type of study at the end of the Presentation section. Epidemiological studies• Observational studies• Narrative reviewsNarrative reviews critically appraise and summarise literature on a common topic area but do not set specific criteria for selecting literature to be included or a specific review protocol. A narrative review draws together major arguments in a field of discourse or provides a significant historical review of an important aspect of physiotherapy. Narrative reviews should be on topics that do not lend themselves to systematic reviews, e.g., examination of the mechanisms underlying a clinical phenomenon. Narrative reviews will almost always be invited and will be considered only if they are written by authors with 
extensive research experience in the field, usually reflected in multiple significant publications. Authors considering submission of a narrative review should first consult the Journal Editor regarding potential suitability of the review for publication. Narrative reviews of intervention, diagnosis, and prognosis will generally not be accepted.

The following types of studies are a low priority: Studies of the reliability or validity of clinical measurement procedures - Surveys of physiotherapy students • Surveys of physiotherapy practice Any survey with a low response rate (less than $70 \%$ )

Submission of these types of studies should be accompanied by a short (less than 100 words) explanation of why the study would be of particular interest to readers of JoP. The Editorial Board will decide, on the basis of this explanation and the abstract, whether the manuscript should be considered for publication. If accepted, such studies will be published as papers of less than 2000 words with no more than one table or figure.

The following types of studies are not accepted:

- Clinical practice guidelinesAlthough the journal is particularly interested in presenting the recommendations of clinical practice guidelines to its readers, clinical practice guidelines are often developed by consensus and may be endorsed by a professional body. This can make it difficult to apply the Journal's normal process of peer review. Therefore, particularly relevant guidelines that have been developed using a rigorous process and endorsed by a high quality professional body, such as NHMRC, will be summarised in the Appraisal section of the journal, but will not be republished. Details of the location where hard or electronic copies of the full guidelines are available will be given in the summary. Pilot studiesPilot clinical trials are those that are not designed to have adequate statistical power. Their purpose is to test the feasibility of an intervention in terms of recruitment and delivery of the intervention, as well as to examine the rate of dropouts. They usually provide information to power a future trial and do not therefore reach firm conclusions.

$\mathrm{http://www}$.journalofphysiotherapy.com/content/authorinfo

\section{1/11/2015 Journal of Physiotherapy}

Manuscript length (not including title page, abstract, references, tables or figure legends) depends on the type of study: - Systematic reviews: up to 5000 words $\cdot$ Clinical trials, experimental and qualitative studies: up to 3500 words• Observational studies: up to 2500 words

Authors may be invited, or in some cases required, to place important supplementary material as electronic addenda (eAddenda) on the JoP web site.

\section{MANUSCRIPT PRESENTATION}

Research manuscripts should consist of a title page, abstract, text, references, tables, and figures. Manuscripts should be prepared with $2.5 \mathrm{~cm}$ margins and a footer containing an abbreviated title, the first author's family name, page number and date. The abstract, introduction, method, results, and discussion should be 1.5 line-spaced, but all other text should be single-spaced. Put a double return between paragraphs. Download the journal's manuscript template.

\section{Title Page}

The title of the manuscript should not be more than 25 words and should be in two parts. Give the main results of the study followed by a colon and the method used, e.g., 'A resource-efficient exercise program after discharge from rehabilitation improves standing ability in people after stroke: a randomised trial'. Download example titles for different research designs.

Then, list all authors and their degrees, positions, institutions, country, and email address. Nominate a corresponding author for the review who is authorised to negotiate and approve editorial revisions, provide his/her title (Professor, Dr, etc.), and give contact details (email address). You may nominate a different corresponding author for publication; provide his/her title (Professor, Dr, etc.) and short 
contact details (department/institution, postal address and email address).

Provide a running head of up to six words. Next, for indexing purposes, select up to five key words from the Index Medicus Medical Subject Headings (MeSH). MeSH Headings can be found on the PubMed MeSH browser at http://www.nlm.nih.gov/mesh/meshhome.html.

List the word count for the abstract and the body of the text, as well as the number of references, tables, and figures.

Finally, list the Ethics Committee(s) that approved the study and the procedures for gaining consent, source(s) of support, acknowledgements, and any competing interests. The statements regarding ethics and consent do not need to be re-stated in the body of the manuscript. Acknowledgments should include statements of important contributions that do not justify authorship. The nature of the contribution should be specified. It is customary to seek permission of people named in the acknowledgments. Download the journal's Title Page template.

\section{Abstract}

An abstract of no more than 250 words is required for all submissions using the headings: Question, Design, Participants, Intervention, Outcome measures, Results, Conclusion, and Trial registration (if appropriate). The results should include estimates of effect sizes and their confidence intervals rather than $p$ values. Abstracts should not contain references. Download examples of abstracts for different research designs

\section{Introduction}

The introduction should justify the aims of the research. Only references essential to understanding these aims should be included. Introductions rarely need to be longer than five paragraphs. At the end of the introduction, list the research questions as given in the Abstract again. Download Research question examples for different research designs

\section{Method}

Use the subheadings: Design; Participants, therapists, centres; Intervention; Outcome measures; and Data analysis, as appropriate to the design of the study. Restrict headings to no more than two levels of importance (i.e., avoid sub-subheadings). Where aspects of the method have been described in other widely-available publications a reference to those publications may suffice, whereas newlydeveloped procedures should be described in more detail.

In the Design section, describe the overall design, especially the timing of intervention and measurement, and any randomisation or blinding procedures.

In the Participants, therapists, centres section, outline the recruitment procedures and the inclusion and exclusion criteria for eligibility of participants, therapists, and centres.

In the Intervention section, give as much detail as necessary so that the intervention could be faithfully replicated by a reader. If this requires extensive material, consider placing some in an Appendix, which can be an electronic-only addendum to the paper.

In the Outcome measures section, state the impairment/activity limitation/participation restriction being collected (e.g., walking) and its measurement with units (e.g., velocity during $10 \mathrm{~m}$ Walk Test in $\mathrm{m} / \mathrm{s}$ ). Other examples are: strength measured as peak isometric elbow extensor torque using handheld dynamometry in $\mathrm{Nm}$, or pain measured as intensity at rest on a $10 \mathrm{~cm}$ VAS in $\mathrm{cm}$. It can be useful to divide outcome measures into those examining impairments vs activity limitations vs participation restrictions. It is only necessary to refer to manufacturers' information for equipment when the precise specifications could be important to interpretation of the study. Information should be placed in a footnote at the end of the text, coded using consecutive, superscripted lower case letters.

In the Data analysis section, outline any a priori power analysis carried out to determine the number of participants needed for the study. Outline any conversions or calculations made with the data. Explain how the research questions are answered by the interpretive tests but do not name the statistical package used if it is widely available. 


\section{Results}

The first subheading should be Flow of participants, therapists, and centres through the study where the numbers at each point in the study are presented as well as baseline characteristics. The remainder of the results should report only the data that answer the research questions and should be organised under subheadings that reflect those questions. Pertinent results should be reported using text and/or tables and/or figures; tables are more useful than figures because exact values are given. Avoid repeating in the text data presented in tables or figures. Do not duplicate data in tables and figures.

When reporting data, be conscious of the precision of the data and only report a meaningful number of decimal places. Usually, report numbers between 0 and 1 to 2 decimal places, between 1 and 10 to 1 decimal place, and above 10 with no decimal place.

All data reported as numbers should also be given as a percentage of the sample (in brackets) rounded off, e.g., 17 (34\%) participants were men. All data reported as means should also be accompanied by the standard deviation (in brackets), e.g., the mean height of participants was $1.53 \mathrm{~m}$ (SD 0.23).

When reporting the results of interpretive tests, report the size of the effect rather than its statistical significance, e.g., 'People with arthritis were twice as likely to sprain their ankle (OR 0.50, 95\% $\mathrm{Cl} 0.25$ to 0.75$)$ ' or 'People after stroke walked $0.65 \mathrm{~m} / \mathrm{s}(95 \% \mathrm{Cl} 0.60$ to 0.70$)$ slower than their age-matched healthy counterparts', but not 'People with asthma were significantly more breathless after exercise $(p$ $=0.02)^{\prime}$.

\section{Discussion}

New and important findings should be emphasised but, as a rule, data already presented in the Method and Results sections should not be repeated. Implications and limitations of the findings and their clinical application should be discussed. The length of the Discussion should be commensurate with the number of important findings; usually it will be less than 750 words. Do not include a separate conclusion at the end of the Discussion.

\section{References}

Only essential references should be cited. Most research will require fewer than 30 references. If the research requires considerably more (e.g., systematic reviews of areas with many clinical trials), references may be provided as supplementary material or eAddenda.

The referencing style used by the journal is the JAMA style, which can be found as a standard referencing style in EndNote, RefWorks, Mendeley, and Zotero. If you use reference management software such as these, please convert your paper to the JAMA style before submission. Journal titles should be abbreviated according to the journals list in PubMed (). Please ensure that all references are complete and presented using numbered style.

\section{Tables}

Tables should appear after the references and each table should start on a separate page. They should be numbered consecutively in the order to which they are referred in the text. A short caption should be given above each table (e.g., 'Table 1. Characteristics of participants.'). Within the table, give the units of outcome measures in brackets and italics, e.g., $(\mathrm{m} / \mathrm{s})$. When reporting counts (frequencies), give percentages in brackets. Use abbreviations for time (i.e., s, $\min , h r$, etc.) and amount (i.e., $k g$, deg $\mathrm{Nm}$, etc.) without a legend explaining them. Where abbreviations for physiotherapy-specific terms are used (e.g., ROM, MCP, etc.), provide a legend below the table. Tables should be presented with a minimum of horizontal lines and no vertical lines. Download examples of tables.

\section{Figures}

Figures should start on a separate page after the tables. They should be displayed at the proposed publication size and numbered consecutively in the order to which they are referred in the text. A short caption should be given below each figure, e.g., 'Figure 1. Mean (SD) effect of posture on forced expiratory volume for the experimental group (closed circles) and the control group (open circles)'. Do not place boxes around figures. Do not put axes on the top and right sides of graphs. Use symbols and/or line types rather than colour to differentiate data. Where several graphs refer to closely-related 
material, present them as separate panels of a single figure labelled A, B, C, etc., and provide one caption explaining what is in each panel. Photographs should be in sharp focus, have simple backgrounds, and be in black and white unless colour is essential to illustrate the point (e.g., MRI).

For publication, photographs should be supplied as digital images saved at a minimum of $300 \mathrm{dpi}$ in .jpg format. Graphs and line drawings generated by commonly-used graphing programs (such as Microsoft Excel) are acceptable. Written permission should be obtained for use of previously published Figures and Tables, and for publication of photographs of recognisable subjects. These documents should be uploaded with the final manuscript once it has been accepted.

\section{Boxes}

When information needs to be listed but is not a table (contains numbers) or a figure (photograph, graph, or flow diagram), then it should be called a Box. Boxes should be numbered consecutively in the order to which they are referred in the text. A short caption should be given above each box (e.g., 'Box 1. Elements of a viable patient education program.') Download examples of boxes formatted to these specifications.

\section{Style}

Manuscripts should be written in simple, direct, and grammatically-correct English. Use Australian/English spelling. Use gender neutral and non-labelling language (e.g., 'People with back pain' rather than 'back pain patients'). When people are enrolled in a trial, use 'participant' rather than 'subjects'. Use capitals (upper case letters) sparingly but capitalise proper nouns. Divisions of the data set are also capitalised (e.g., 'Group 1' or 'Stage 2'). See previous issues for other specific aspects of JoP style.

Click below for the guidelines and examples available for the following types of studies: • Systematic Review guidelines• Systematic Review examples• Clinical Trial guidelines

- Clinical Trials examples• Qualitative Study guidelines• Papers reporting the results of questionnaires guidelines

\section{MANUSCRIPT SUBMISSION}

All manuscripts, correspondence and editorial material for publication should be submitted online via the Elsevier Editorial System at http://ees.elsevier.com/jphys. Authors first 'create a new account' (i.e., register) by following the instructions at the website, and

using their own email address and selected password. Authors can then upload manuscripts containing text, tables, images (figures), and any supplementary material (eAddenda). You will be guided stepwise through the creation and uploading of the various files. The entire peer-review process is managed electronically to ensure timely review and publication. All correspondence, including notification of the Editor's decision and requests for revision, takes place by email and via the Author's homepage, removing the need for a hard-copy paper trail.

Note: articles submitted for the review process may be edited after acceptance to conform to journal standards. For this an 'editable' file format is necessary; we prefer a Word file. Ensure that all track changes have been accepted and the reviewing function is turned off. Retain identical hard and electronic copies of the manuscript and all illustrative material. Manuscripts will be acknowledged on receipt. Those which are not presented according to Journal of Physiotherapy guidelines will be returned to the author for amendment. Although Elsevier can process most file formats, should your electronic file prove to be unusable, the article will be typeset from the hardcopy printout and particular care should be taken to check the proofs.

Submission of an article implies that the work described has not been published previously (except in the form of an abstract or as Part of a published lecture or academic thesis), that it is not under consideration for publication elsewhere, that its publication is approved by all authors and tacitly or explicitly by the responsible authorities where the work was carried out, and that, if accepted, it will not be published elsewhere in the same form, in English or in any other language, without the written consent of the Publisher.

\section{Compulsory Authorship Form}


JoP policy on Authorship is based on the guidelines for authorship in the International Committee of Medical Journal Editors Uniform Requirements for Manuscripts Submitted to Biomedical Journals 2004 (www.icmje.org) which states that 'authorship should be based on 1) substantial contributions to conception and design, or acquisition of data, or analysis and interpretation of data; 2) drafting the article or revising it critically for important intellectual content; and 3) final approval of the version to be published. Conditions 1, 2, and 3 must all be met. Acquisition of funding, the collection of data, or general supervision of the research group, by themselves, do not justify authorship'. Manuscript submission, and completion of the online Authorship form signifies that all authors satisfy the ICMJE criteria for authorship.

\section{OPEN ACCESS}

This journal is open access for Editorials and peer-reviewed Research Articles where they will be immediately and permanently free for everyone to read and download. Permitted reuse is defined by the following Creative Commons user license:

Creative Commons Attribution-NonCommercial-NoDerivatives (CC BYNC-ND): for non-commercial purposes, lets others distribute and copy the article, and include in a collective work (such as an anthology), as long as they credit the author(s) and provided they do not alter or modify the article.

Open Access of Editorials and Research Articles is sponsored by the Australian Physiotherapy Association.

\section{PEER REVIEW}

Research manuscripts are subject to peer review. If the Journal Editor considers that the manuscript is likely to be of interest to readers and is potentially publishable, the manuscript is sent to two reviewers. Reviewers will usually have specific expertise in the field and a record of recent publication in peer-reviewed journals. Reviewers are asked to advise the Journal Editor if the manuscript is credible and of importance to the physiotherapy profession; they are also asked to comment on the manuscript's validity, relevance, clarity, and conciseness. They are asked to provide their reports within four weeks of receipt of the manuscript.

Reviewers are asked to consult checklists where appropriate. Specifically, reviewers of randomised controlled trials are asked to consult the CONSORT e-checklist, reviewers of systematic reviews are asked to consult the PRISMA statement, and reviewers of studies of the accuracy of diagnostic tests are asked to consult the STARD checklist. These checklists can be found at http://www.consort-statement.org/resources/downloads

The Journal Editor considers the reviewers' comments and decides if the manuscript is to be accepted in its current form, accepted subject to minor revisions, potentially publishable but requiring significant revision, or not suited to publication in JoP. Authors are provided with the reviewers' comments, sometimes with additional comments made by the Scientific Editor, and are informed of the decision. Authors of manuscripts requiring revision are invited to consider and respond to the comments made by the reviewers and the Journal Editor, revise the manuscript accordingly, and re-submit. Usually the revised manuscript is returned to the original reviewers for further comment. Some manuscripts undergo several rounds of review before a final decision (accept or reject) is made.

Usually authors hear within 7-10 days if the journal Editor decides that the submission is not suitable for publication in JoP. Time to first decision after review (accept, revise with guarantee, revise without guarantee, or reject) is generally no more than 2 months from submission. Once accepted, manuscripts will go into production and be made available online as an article in press. They undergo extensive editing to improve clarity and comply with JoP style. Author(s) are given the opportunity to review the accuracy of the edited manuscript at proof stage prior to publication. Authors are provided with a .PDF of the final version.

\section{TRIAL PROTOCOLS}

Journal of Physiotherapy accepts research protocols for major prospective studies. An abstract of the protocol will be published in the journal, supported by the full version of the protocol available as Appraisal content from the journal website.

To be eligible for consideration the study must have received nationally competitive funding. Submissions will be reviewed by the Protocol Section Editor, and by members of the journal's Editorial Board. The protocols we select for publication need to meet several high standards including that the trial will be likely to directly influence how physiotherapists practice, and/or the trial will significantly enhance understanding of conditions treated by physiotherapists. 
full study protocol and an abstract prepared according to the Journal of Physiotherapy Protocol template; and upload of a completed Authorship statement.

\section{EDITORIALS}

Journal of Physiotherapy publishes one or two editorials on scientific or professional issues of physiotherapy practice in each issue. Editorials are usually commissioned; however, anyone wishing to write an editorial should contact the Journal Editor at ScientificEditorJoP@physiotherapy.asn.au for discussion about the topic. Editorials should be no more than 2000 words with a maximum of three authors (unless agreed with the Journal Editor before the work begins) and 20 references. Commissioned editorials are not formally peer reviewed, but may be subject to informal review. Non-commissioned editorials will be formally peer reviewed.

\section{CORRESPONDENCE}

Correspondence to Journal of Physiotherapy should be uploaded via the Elsevier Editorial System. Correspondence is reviewed by the Journal Editor and may be edited. Generally, correspondence falls into two categories: letters challenging physiotherapy assumptions about practice, and letters commenting on papers published in the journal (particularly welcome). In general, such letters should be submitted soon after publication of the paper they refer to. Authors of the papers will usually be invited to reply.

All letters should be no more than 500 words and should contain no more than five references.

Copyright ( $\odot 2015$ Elsevier Inc. All rights reserved. | Privacy Policy | Terms \& Conditions | About Us | Help \& ContactThe content on this site is intended for health professionals.Advertisements on this site do not constitute a guarantee or endorsement by the journal, Association, or publisher of the quality or value of such product or of the claims made for it by its manufacturer.

\section{QUALIS}

\section{Periódicos}

\begin{tabular}{|l|l|l|c|}
\hline ISSN & Título & \multicolumn{1}{|l}{ Área de Avaliação } & Classificação \\
\hline $1836-9553$ & $\begin{array}{l}\text { Journal of } \\
\text { Physiotherapy }\end{array}$ & EDUCAÇÃO FÍSICA & B1 \\
\hline $2240-4929$ & $\begin{array}{l}\text { Italian Journal of } \\
\text { Physiotherapy }\end{array}$ & ENGENHARIAS IV & B1 \\
\hline $1836-9553$ & $\begin{array}{l}\text { Journal of } \\
\text { Physiotherapy }\end{array}$ & MEDICINA II & B3 \\
\hline $0004-9514$ & $\begin{array}{l}\text { Australian Journal of } \\
\text { Physiotherapy }\end{array}$ & PSICOLOGIA & \\
\hline
\end{tabular}


Archives of Physical Medicine and Rehabilitation - fator de impacto 2,69

\section{Archives of Physical Medicine and Rehabilitation}

\section{INTRODUCTION}

-Types of papers

\section{BEFORE YOU BEGIN}

All Content

Search

-Submission • Referees•Revisions • Resubmissions •Additional information PREPARATION

-Your Paper Your Way •NEW SUBMISSIONS •References•Formatting requirements $\cdot$ Peer Review

•REVISED SUBMISSIONS • Subdivision·Title Page•Abstract

-Keywords •Abbreviations

Advanced Search

-Main Manuscript $\cdot$ Graphical abstract $\cdot$ Highlights•Units

-Footnotes•Artwork•Tables•Supplementary data•Suppliers•References•Submission checklistAFTER ACCEPTANCE·Use of the Digital Object Identifier •Proofs

•AudioSlides • OffprintsAUTHOR INQUIRIES

-Ethics in Publishing $\overline{\cdot H u m a n}$ and Animal Rights $\overline{\text { Conflict of Interest }} \overline{\text { Submission declaration and verification }}$ -Authorship·Clinical trial·NEW - Reporting Guidelines and Checklists $\cdot$ Copyright $\cdot$ Role of the funding source $\cdot$ Funding body agreements and policies • Page charges•Open access•Green open access•Language (usage and editing services)

Archives of Physical Medicine and Rehabilitation publishes original articles that report on important trends and developments in physical medicine and rehabilitation and in the wider interdisciplinary field of rehabilitation. Archives of Physical Medicine and Rehabilitation brings readers authoritative information on the therapeutic utilization of physical and pharmaceutical agents in providing comprehensive care for persons with disabilities and for chronically ill individuals. Archives began publication in 1920, publishes monthly, and is the official journal of the ACRM | American Congress of Rehabilitation Medicine. Its content is cited more often than any other rehabilitation journal. 


\section{Types of papers}

Original Research: Present new and important basic and clinical information, extend existing studies, or provide a new approach to a traditional subject. Manuscripts should be limited to 3000 words of text (Introduction through Conclusions). Figures, tables, and references should be limited to the number needed to clarify, amplify, or document the text.

Brief Reports: Provide preliminary communications of new data, research methods, brief case studies of interest, new ideas, and techniques. Manuscripts should be limited to 1500 words of text (or 1200 words plus 1-2 figures or tables, Introduction through Conclusions), and no more than 10 references.

The Archives will not consider case reports for publication. Please do not submit them.

Commentaries (by Invitation): Focus on issues in physical medicine and rehabilitation. Manuscripts should be limited to 2000 words of text (Introduction through Conclusions). The Editorial Board reserves the right to ensure that the author is qualified, through education and professional experience, to write knowledgeably and appropriately about a particular subject before accepting a Commentary for publication. The Editorial Board will choose the author(s) for Invited Commentaries and the author(s)' identity will be anonymous until publication. Authors of the subject article may submit a response for a subsequent issue.

Editorials: Editorials published in Archives may only be written by the elected officers of ACRM, or by members of the Editorial Board. Prior to publication, all editorials are approved by the Editorial Board's Executive Committee. Editorials do not represent the opinions or positions of ACRM or the Editorial Board. Editorials should be limited to 1000 words of text.

Information/Education: The ACRM Communications Committee has developed a new feature, Information/Education Pages, which appear in the Organization News section of Archives. These fact sheets are printed as tear-out pages. They are designed to provide consumer-friendly information on topics relevant to rehabilitation medicine, including basic background or overview, similar to a Wikipedia entry, or brief how-to suggestions. They are targeted toward people with disabilities, their caregivers, or clinicians; and are designed so that a practitioner can tear out and copy, or download the pages, to make them available to patients and caregivers.

Authors are invited to submit Information/Education Page manuscripts or proposals to the Archives' Editorial Office

(ArchivesMail@archives.acrm.org). The ACRM Communications Committee will assess subject matter, content, and target reading level then provide feedback on suitability and instructions on how to proceed directly to the author. Note that this should not be considered an official peer review of the content.

Letters to The Editor: Letters are published at the discretion of the Editorial Board and should be directly related to the published article on which it comments. Letters may not reference unpublished studies or reference "in press" studies that are not publicly available. The Editorial Board reserves the right to solicit a response from the authors of the cited article. Letters must be limited to roughly 500 words of text, 1 table, and no more than 5 references.

Measurement Tools: These instrument summaries, which appear in the Organization News section of Archives, are designed to facilitate the selection of outcome measures by trained clinicians. The information contained in this summary represents a sample of the peer-reviewed research available at the time of the summary's publication. The information contained in these summaries does not constitute an endorsement of the instrument for clinical practice. The views expressed are those of the summary authors and do not represent those of authors' employers, instrument owner(s), the Archives, the Rehabilitation Measures Database or the United States Department of Education. The Rehabilitation Measures Database and Instrument Summary tear-sheets are funded by the National Institute on Disability and Rehabilitation Research, United States Department of Education through the Rehabilitation Research and Training Center on Improving Measurement of Medical Rehabilitation Outcomes (H133B090024) and Improving Measurement of Medical Rehabilitation Outcomes (H133B090024). Authors are invited to submit Measurement Tools through the Archives' submission platform.

Review Articles (Meta-Analyses): The Editorial Board invites proposals for state-of-the-art review articles. Manuscripts should be limited to 5000 words of text (Introduction through Conclusions), exclusive of references. The Archives strongly prefers systematic reviews of the literature. 
Special Communications: Provide information or an objective analysis of issues in physical medicine and rehabilitation that does not qualify as a research or clinical paper or commentary. Manuscripts are peer reviewed and should be limited to 5000 words of text, exclusive of references.

\section{Ethics in Publishing}

\section{Authorship}

Manuscripts should have no more than 8 authors; a greater number requires written justification. The order of authorship is a joint decision of the coauthors. Archives follows the Recommendations for the Conduct, Reporting, Editing, and Publication of Scholarly Work in Medical Journals guidelines ${ }^{1}$, which state authorship credit should be based only on substantial contributions to (1) conception and design, or acquisition of data, or analysis and interpretation of data, (2) drafting the article or revising it critically for important intellectual content, and (3) final approval of the version to be published. Conditions 1, 2, and 3 must all be met. Participation solely in the acquisition of data does not justify authorship, nor does general supervision of the research group. Archives may require authors to justify the assignment of authorship. Increasingly, multicenter trials are attributed to a corporate author. All members of the group who are named as authors, either in the authorship position below the title or in a footnote, must fully meet the criteria for authorship as defined above. Group members not meeting these criteria should be listed, with their permission, in the Acknowledgments. Acknowledgments to other investigators for advice or data must be documented by written authorization specifically granting permissions to the authors.

Changes in authorship: After a manuscript has been submitted, any addition, deletion, or change to the order of the authors must be submitted in writing ${ }^{2}$ to the Editorial Office

(ArchivesMail@archives.acrm.org). This written statement, explaining the change and listing the old and new author orders, must be submitted with all authors copied (including those who have been removed, if applicable). The corresponding author should instruct all copied authors to respond with their approval of the change in author order. Failure to respond or failure of all authors to agree to the change may lead to suspension of review/publication of the article.

\section{Authorship/Copyright form and ICMJE form}

Archives requires 2 forms. For both new submissions and revisions, the peer-review process will not begin until these documents are completed correctly and submitted as per the instructions below.

Step 1: Archives requires the author submitting the manuscript to complete and upload the electronic version of the journal's Authorship Form and Copyright Assignment available here: http://cdn.elsevier.com/promis_misc/dscaoriginalsubmission-2014.pdf. The author submitting the manuscript must also upload the separate ICMJE Forms for Disclosure of Potential Conflicts of Interest. By this act, the author submitting the manuscript will serve as the guarantor for all coauthors in presenting accurate disclosures for the author group. The guarantor is expected to consult with all coauthors about the disclosures he/she provides. Any disclosure (i.e. actual or perceived conflict of interest) must be described on the title page of the manuscript.

Step 2: At the point an editor seeks revision of a manuscript, Archives will require, with submission of the revised manuscript, original copies from all coauthors of the ICMJE form. Review of the revision will not commence until the editors have fully and accurately received the completed ICMJE forms from all coauthors. The editors expect the guarantor's group disclosure at submission to be consistent with the individual disclosures received at the revision stage. A written explanation will be required if this is not the case. If it is not possible to provide ICMJE forms from all co-authors at the revision stage, please contact the Editorial Office (ArchivesMail@archives.acrm.org) for alternative instructions.

Conflict of Interest: Authors must reveal to the Editorial Board any conflicts of interest that the Editorial Board or the Archives readers would reasonably consider relevant to the research, analysis, or interpretation presented in the manuscript. The Board will hold this information in confidence, unless the study is accepted and, in the Board's judgment, readers need to be made aware of the general nature of this possible conflict. In this case, a general description of the conflict will be published with the article.

Device Status: The submitting author must include in the title page to the manuscript any applicable Device Status Statement, as selected in the Authorship and Copyright Assignment form. The statement does not affect the decision to publish a manuscript; that decision is made solely on the basis of the 
article's content and its value to the journal's readers. The selected statement may be published with the article.

\section{Redundant or Duplicate Publication}

Archives, as a primary source periodical, does not consider for publication material that already has been reported in a published article or is described in a paper submitted or accepted for publication elsewhere, in any print or electronic media. Abstracts (250-300 words) of preliminary research findings that are published in conference proceedings are not considered previous publications (except for submissions to the Brief Reports category). This policy does not usually preclude consideration of a manuscript that has been rejected by another journal or of a complete report that follows publication of a preliminary report, usually in the form of an abstract (250-300 words). Press reports on papers presented at a meeting will not usually be considered prior publication, but such reports should not be amplified by additional data or copies of tables and illustrations. Authors submitting manuscripts to Archives must include in their cover letter an explanation of any prior publication (published article, article in press, manuscript under review, published abstract) of the same or substantially similar work, and should explain any circumstances that might cause the Editorial Board to believe that the manuscript may have been published elsewhere (e.g. similar titles). Authors must state whether the paper includes subjects about whom a previous report has been published. Authors must include an electronic copy (upload as Related (un)published manuscripts and/or meeting abstracts) of any published article or an electronic copy of any submitted manuscript that deals in any respect whatsoever with the same patients, same animals, same laboratory experiment, or same data-in part or in full - as are being reported in the manuscript they submit to Archives.

Duplicate Publication: Duplicate publication is the publication of the same paper or substantially similar papers in any medium. Publication more than once of the same study results, whether or not the wording is the same, is rarely justified. Articles previously published in another language will not be considered for publication. The Editorial Board will take appropriate disciplinary action against authors who engage in duplicate publication of the same or substantially similar data. The Editorial Board reserves the right to consult with other journals about the content of the papers in question. Further, the Editorial Board (1) may return manuscripts prior to the review process, (2) may decide not consider any manuscripts from the author(s) for a period of time, (3) may announce publicly in Archives that the authors have submitted a previously published article, or (4) may refer the incident to COPE (The Committee on Publication Ethics) for discussion or advice, or (5) may take any combination of these actions. If the paper is accepted and published before evidence of duplication is discovered, the Editorial Board will announce the duplication in Archives and/or will request that the authors write a letter acknowledging the duplicate publication. The Editorial Board will notify appropriate institutions, ranging from national databases to the authors' departments or university administrators, at its discretion.

Preliminary Release: Preliminary release, usually to the media, of scientific information described in a study that has been accepted by Archives but not yet published violates the copyright agreement between the authors and the journal. The Editorial Board may approve advance release of data (e.g. to warn the public of health hazards) in certain situations. Authors should contact the Editorial Office (ArchivesMail@archives.acrm.org) to discuss embargoes, as embargoes will preempt conditions of preliminary release.

Simultaneous Submission: Authors should not submit the same manuscript simultaneously to more than 1 journal. If the Editorial Board learns of possible simultaneous submission, it reserves the right to consult with the other journal that received the manuscript. Further, the Editorial Board may return the manuscript prior to the review process, or may reject it without regard to peer reviewer recommendations and may decide not to consider any studies from the author(s) for a period of time.

\section{Human and Animal Rights}

If relevant, a statement must be included in the body of the manuscript that human experimentation was approved by the local institutional review board or conforms to the Helsinki Declaration ${ }^{3}$, as stated in the section Manuscript Preparation, Methods. Also that guidelines for the care/use of nonhuman animals or other species, approved by the institution, were followed as indicated in the Methods. The species must be named in the Title, Abstract, and Methods section.

\section{Conflict of Interest}


The Archives utilizes two forms:(1) the ICMJE Form for Disclosure of Potential Conflicts of Interest, and (2) the Archives' Authorship Form \& Copyright Assignment

During original submission, the corresponding author completes and uploads both the Authorship Form \& Copyright Assignment and the ICMJE form. If a revised paper is submitted, all authors must complete the ICMJE form.

If any of the authors do have a conflict of interest, this should be clearly explained on the title page of the manuscript.

Please see the ICMJE author responsibilities regarding conflicts of interest ( http://www.icmje.org/recommendations/browse/roles- and-responsibilities/author-responsibilities-conflicts-of-interest.html). It is important to note that a conflict of interest can be actual or perceived.

\section{Submission declaration and verification}

Submission of an article implies that the work described has not been published previously (except in the form of an abstract or as part of a published lecture or academic thesis or as an electronic preprint, see http://www.elsevier.com/sharingpolicy), that it is not under consideration for publication elsewhere, that its publication is approved by all authors and tacitly or explicitly by the responsible authorities where the work was carried out, and that, if accepted, it will not be published elsewhere in the same form, in English or in any other language, including electronically without the written consent of the copyright-holder. To verify originality, your article may be checked by the originality detection service CrossCheck http://www.elsevier.com/editors/plagdetect.

\section{Authorship}

Authors have read the submitted manuscript and vouch for its accuracy. All authors have participated sufficiently in the conception and design of this work and the analysis of the data (where applicable), as well as the writing of the manuscript to take public responsibility for its content. If any author (or group of authors) listed cannot verify substantial contribution, the author's name should be moved to the acknowledgment section. If requested, authors shall produce the data on which the manuscript is based for examination by Archives or its assignees.

Authors warrant the manuscript is original and its essential substance, tables, or figures have not been previously published in part or in whole. The manuscript or one with substantially similar content under declared authorship or the data within it has not been accepted for publication elsewhere and it is not presently under review by any other publisher. The manuscript will not be submitted for publication elsewhere until a decision has been made on its acceptability for publication in Archives. This restriction does not apply to brief abstracts or press reports published in connection with scientific meetings.

\section{Clinical trial}

While there may be occasional exceptions, the Archives is committed to the need for clinical trial reports to be accompanied by adequate periods of follow-up. A lack of sufficient follow-up may be detrimental to a paper's acceptance.

Beginning January 1, 2016 all manuscripts reporting clinical trials must be registered before submission. For trials that are underway and are already enrolling patients, registration will be retrospective. This is an interim step that will end January 1, 2017. At that time, the Archives will only consider clinical trials that have been registered before the first patient is enrolled.

For our purposes, a clinical trial is defined as "any research study that prospectively assigns human participants or groups of humans to one or more health-related interventions to evaluate the effects on health outcomes" ( http://www.who.int/ictrp/en). Thus, cohort and retrospective studies without an intervention do not require registration, and neither do observational studies of clinical care. However, studies of human subjects with prospective assignment of an intervention by the investigators, regardless of the size of the trial or method of assignment, must be registered.

\section{NEW - Reporting Guidelines and Checklists}

To ensure a high and consistent quality of research reporting, original research articles must contain 
sufficient information to allow readers to understand how a study was designed and conducted. For review articles, systematic or narrative, readers should be informed of the rationale and details behind the literature search strategy.

To achieve this goal, Archives requires that authors upload a completed checklist for the appropriate reporting guideline during original submission. Taking the time to ensure your manuscript addresses basic reporting prerequisites will greatly improve your manuscript, and enhance the likelihood of publication. These checklists serve as a guide for the editors and reviewers as they evaluate your paper.

The EQUATOR Network ( www.equator-network.org) is an excellent resource for key reporting guidelines, checklists, and flow diagrams. These guidelines should be especially useful for Archives' authors. Click on the checklist that applies to your manuscript, download it to your computer, fill it out electronically, "save as," and upload it with your manuscript when you submit. Links to mandatory flow diagrams also are provided. Below are the most commonly used checklists but please note that the Equator Network provides many others (e.g. TRIPOD, SRQR, etc.) and it is up to the authors to select the one most appropriate for their study.

Randomized Controlled Trials - CONSORT - Consolidated Standards of Reporting TrialsObservational Studies - STROBE - Strengthening the Reporting of Observational studies in EpidemiologySystematic Review of Controlled Trials - PRISMA - Preferred Reporting Items for Systematic Reviews and Meta-Analyses Study of Diagnostic accuracy/assessment scale - STARD Standards for the Reporting of Diagnostic Accuracy Studies

During the submission process when you are prompted to state which checklist is needed please check the appropriate box for your manuscript or check Not Applicable if your paper is a Commentary, Letter to the Editor, etc. Then the system will allow you to select the file type and upload the appropriate checklist and flow diagram. IT IS PERMISSIBLE TO ADD A COLUMN OR SPACE TO THE CHECKLIST THAT SPECIFIES WHERE IN THE MANUSCRIPT EACH COMPONENT HAS BEEN FOLLOWED AND USE THAT FOR YOUR UPLOAD. YOU MAY NEED TO DO THIS FOR STROBE AS WELL AS OTHERS. A MODIFIED STROBE FORM IS AVAILABLE HERE.

\section{Copyright}

Upon acceptance of an article, authors will be asked to complete a 'Journal Publishing Agreement' (for more information on this and copyright, see http://www.elsevier.com/copyright). An e-mail will be sent to the corresponding author confirming receipt of the manuscript together with a 'Journal Publishing Agreement' form or a link to the online version of this agreement.

Subscribers may reproduce tables of contents or prepare lists of articles including abstracts for internal circulation within their institutions. Permission of the Publisher is required for resale or distribution outside the institution and for all other derivative works, including compilations and translations (please consult http://www.elsevier.com/permissions). If excerpts from other copyrighted works are included, the author(s) must obtain written permission from the copyright owners and credit the source(s) in the article. Elsevier has preprinted forms for use by authors in these cases: please consult http://www.elsevier.com/permissions.

For open access articles: Upon acceptance of an article, authors will be asked to complete an 'Exclusive License Agreement' (for more information see http://www.elsevier.com/OAauthoragreement). Permitted third party reuse of open access articles is determined by the author's choice of user license (see http://www.elsevier.com/openaccesslicenses).

\section{Author rights}

As an author you (or your employer or institution) have certain rights to reuse your work. For more information see http://www.elsevier.com/copyright.

\section{Role of the funding source}

You are requested to identify who provided financial support for the conduct of the research and/or preparation of the article and to briefly describe the role of the sponsor(s), if any, in study design; in the collection, analysis and interpretation of data; in the writing of the report; and in the decision to submit the article for publication. If the funding source(s) had no such involvement then this should be stated. 


\section{Funding body agreements and policies}

Elsevier has established a number of agreements with funding bodies which allow authors to comply with their funder's open access policies. Some authors may also be reimbursed for associated publication fees. To learn more about existing agreements please visit

http://www.elsevier.com/fundingbodies. Page chargesArchives has no page charges.Open access

This journal does not ordinarily have publication charges; however, authors can now opt to make their articles available to all (including non-subscribers) via the ScienceDirect platform, for which a fee of US $\$ 3000$ applies (for further information on open access see

http://www.elsevier.com/openaccessoptions). Please note that you can only make this choice after receiving notification that your article has been accepted for publication, to avoid any perception of conflict of interest. The fee excludes taxes and other potential costs such as color charges. In some cases, institutions and funding bodies have entered into agreement with Elsevier to meet these fees on behalf of their authors. Details of these agreements are available at http://www.elsevier.com/fundingbodies. Authors of accepted articles, who wish to take advantage of this option, should complete and submit the order form (available at

http://www.elsevier.com/locate/openaccessform.pdf). Whatever access option you choose, you retain many rights as an author. More information can be found here: http://www.elsevier.com/copyright.

Your publication choice will have no effect on the peer review process or acceptance of submitted articles.

\section{Green open access}

Authors can share their research in a variety of different ways and Elsevier has a number of green open access options available. We recommend authors see our green open access page for further information ( http://elsevier.com/greenopenaccess). Authors can also self-archive their manuscripts immediately and enable public access from their institution's repository after an embargo period. This is the version that has been accepted for publication and which typically includes author-incorporated changes suggested during submission, peer review and in editor-author communications. Embargo period: For subscription articles, an appropriate amount of time is needed for journals to deliver value to subscribing customers before an article becomes freely available to the public. This is the embargo period and it begins from the date the article is formally published online in its final and fully citable form. This journal has an embargo period of 12 months. Language (usage and editing services)

Please write your text in good English (American or British usage is accepted, but not a mixture of these). Authors who feel their English language manuscript may require editing to eliminate possible grammatical or spelling errors and to conform to correct scientific English may wish to use the English Language Editing service available from Elsevier's WebShop ( http://webshop.elsevier.com/languageediting/) or visit our customer support site ( http://support.elsevier.com) for more information.

\section{Submission}

Manuscripts must be submitted through the journal's online system at http://ees.elsevier.com/archives-pmr. The review process will not begin until authors have complied completely with the submission requirements. Compliance includes submission of separate documents in the following order: (1) cover letter; (2) title page, including acknowledgments and explanation of any conflicts of interest; (3) main text file (manuscript without author identifiers) including a structured or standard abstract, keywords, list of abbreviations, body of the text, suppliers' list, references, figure legends; (4) figures; (5) tables; (6) appendices; (7) supplementary files; (8) checklist; (9) ICMJE Form for Disclosure of Potential Conflicts of Interest; and (10) the Archives Authorship Form \& Copyright Assignment.

\section{Referees}

All submissions will be screened by editors to determine their suitability for further review. Manuscripts that are approved for review will be evaluated by at least one recognized expert in the particular subject matter. Biostatistical review may be obtained. Peer reviewers' assessments are referred to a member of the Editorial Board, who may also critique the manuscript. The assigned Editorial Board Member will then make a final decision and communicate with the corresponding author via e-mail. 
Decisions are typically communicated within 60 days after the manuscript has been approved for peer review. All reviews are conducted in a double-blind fashion.

Letters to the Editors and Editorials are generally evaluated by an editorial committee, however, external reviews may also be sought.

Published annually without peer review are the ACRM | American Congress of Rehabilitation Medicine presidential address and the John Stanley Coulter Lecture. The Editorial Board does not peer review the published abstracts of posters, platform presentations of scientific papers, or audiovisual materials presented at the ACRM annual meeting. Archives also publishes the official documents of ACRM. These documents are not peer reviewed by Archives and include position papers and other materials approved by the ACRM.

\section{Revisions}

When submitting your revised manuscript, at the request of the Editorial Board, please include a document, separate from your cover letter, itemizing your response to each of the suggested revisions and any other changes you have made. Use consecutive line numbering in the text and cite line numbers for each change. In addition, highlight each change in the revised manuscript. You will upload this document in the file upload step as the "Detailed Response to Reviewers." Please note that this file should be blinded and should not include author names or institutional letterhead.

If revisions are not received within the time specified in the decision e-mail, the manuscript file will be closed. A revision received after a file has been closed will be handled as a new submission. An extension beyond the deadline may be granted at the Editorial Board's discretion, but only in extenuating circumstances, given the editors' commitment to prompt publication.

Submission of a revised manuscript includes submission of separate documents in the following order: (1) cover letter; (2) title page, including acknowledgments and explanation of any conflicts of interest; (3) main text file with highlighted changes, including an appropriate (structured or standard) abstract, keywords, list of abbreviations, body of the text, suppliers' list, references, figure legends; (4) a clean copy of the main text file with no highlighted changes, including an appropriate abstract, keywords, list of abbreviations, body of the text, suppliers' list, references, figure legends; (5) figures; (6) tables; (7) appendices; (8) supplementary files; (9) checklist; (10) ICMJE Form for Disclosure of Potential Conflicts of Interest for each author; and (11) Archives Authorship Form \& Copyright Assignment.

\section{Resubmissions}

From time to time an author may receive a decision of "Reject-Resubmit" on their original submission. This is a reject but grants the author the opportunity to revise and resubmit their work under a new manuscript number at any time. The resubmission will be linked to the original submission but there will be no expectation of acceptance. The resubmission will be treated as new.

To submit a resubmission authors should note the following:1. Select RESUBMISSION as the article type.2. In your cover letter, please 1) reference this manuscript ID number and include an itemized list of the revisions. 2) Use line numbering in the text and reference the revisions made by page and line number in the cover letter. 3) Highlight changes made in one copy of the manuscript text. Submit another copy with all changes accepted and not highlighted. Please add "marked copy" to the file name of the highlighted version and "clean copy" to the file name of the clean version. Submit both clean and highlighted copies under the category titled Manuscript without author identifiers. Both should remain blinded for the review process.

\section{Additional information}

Unless author(s) notify the Editorial Office of alternate preferences, all accepted articles are posted online within 5 business days of release to production. Author(s) should notify the Editorial Office immediately with any requests to delay posting. This posted version will include a fully citable PDF of the author's accepted files, and will be submitted to PubMed. Supplementary material(s), such as raw data, videos, etc., will not be included. Supplementary materials will be included when the article is typeset and published on the Articles in Press platform or in the monthly print/online issue of the journal.

Manuscripts accepted for publication are subject to editing during the production process. Journal 
style is based on the current AMA Manual of Style. The manuscript will be typeset and the designated corresponding author will receive page proofs for approval. Proofs must be returned to Elsevier by the corresponding author within 48 hours of receipt, as outlined in the e-mail instructions accompanying the proofs.

All accepted manuscripts become the permanent property of Archives and may not be published elsewhere without written permission from the publisher.

\section{Reprints}

Reprint order forms are provided to authors by e-mail in a downloadable PDF format. The reprint form is sent with an e-mail acknowledgment to the author from Elsevier confirming receipt of the accepted manuscript. Reprint orders should be submitted within 15 days to ensure delivery within 6 weeks after publication. Archives does not provide complimentary reprints.

\section{Appeal Process}

Authors may appeal final decisions to the Editor-in-Chief of Archives. This appeal must: (1) be submitted in writing, (2) rebut the negative decision, and (3) be submitted within 30 days after the decision is rendered. Consideration of the appeal will be based on the appeal letter and the version of the manuscript that was peer reviewed. The Editor-in-Chief will assign the appeal to an Editorial Board member for review. The decision from the appeal is final.

Authors should prepare manuscripts according to the "Recommendations for the Conduct, Reporting, Editing, and Publication of Scholarly Work in Medical Journals" 1 as developed by the International Committee of Medical Journal Editors. The Requirements are available at http://www.icmje.org.

\section{Document Formatting}

Manuscripts must be double-spaced throughout, including the title page, abstract, text, acknowledgments, references, individual tables, and legends. Use only standard 12-point type and spacing. Use unjustified, flush-left margins. Number the pages of the text consecutively. Put the page number in the upper or lower right-hand corner of each page. Number each line on each page of the text to facilitate peer review.

Authors should format manuscripts for specific attributes such as italics, superscripts/subscripts, and Greek letters. The coding scheme for each such element must be consistent throughout the file.

Text Style: Enter only 1 space between words and sentences. Leave 1 blank line between paragraphs. Leave 2 blank lines between headings and text.

\section{Your Paper Your Way}

As part of the Your Paper Your Way service, at initial submission you may choose to submit your new manuscript as a single file to be used in the refereeing process. This can be a PDF file or a Word document, in any format or lay-out that can be used by referees to evaluate your manuscript. It should contain high enough quality figures for refereeing. If you prefer to do so, you may still provide all or some of the source files at the initial submission. Please note that individual figure files larger than 10 MB must be uploaded separately. If your paper is accepted, you will you be requested, at the revision stage, to put your paper in the correct format by supplying individual files for the manuscript, tables, figures, etc. and any other items required for the publication of your article. To find out more, please read the rest of the Preparation section.

\section{NEW SUBMISSIONS}

Submission to this journal proceeds totally online and you will be guided stepwise through the creation and uploading of your files. The system automatically converts your files to a single PDF file, which is used in the peer-review process.

\section{References}

There are no strict requirements on reference formatting at initial submission. References can be in any style or format as long as the style is consistent. Where applicable, author(s) name(s), journal title/book 
title, chapter title/article title, year of publication, volume number/book chapter and the pagination must be present. Use of DOI is highly encouraged. The reference style used by the journal will be applied to the accepted article by Elsevier at the proof stage. Note that missing data will be highlighted at proof stage for the author to correct.

\section{Formatting requirements}

There are no strict formatting requirements for articles at initial submission (for requirements for revised submissions, please see REVISED SUBMISSIONS section below) but all manuscripts must contain the essential elements needed to convey your manuscript, for example Abstract, Keywords, Introduction, Methods, Results, Conclusions, Artwork and Tables with Captions.If your article includes any Videos and/or other Supplementary material, this should be included in your initial submission for peer review purposes.Divide the article into clearly defined sections.

Please ensure the text of your paper is double-spaced - this is an essential peer review requirement.

Figures and tables embedded in text - Your Paper Your Way

If you choose the Your Paper Your Way option when submitting your manuscript for the first time, please ensure the figures and the tables included in the single file are placed next to the relevant text in the manuscript, rather than at the bottom or the top of the file.

\section{Peer Review}

Archives uses a double-blind peer-review process. The blinded submission should be submitted in a word document and should begin with a title followed by the abstract, keywords, list of abbreviations, body of the text, references, figure legends, and any relevant suppliers' list.

\section{REVISED SUBMISSIONS}

Please note if you submitted your original manuscript following the Your Paper Your Way format you will now need to put the paper in the correct format by supplying individual files for the manuscript, tables, figures, etc. and any other items required for the publication of your article.

\section{Use of word processing software}

Regardless of the file format of the original submission, at revision you must provide us with an editable file of the entire article. Keep the layout of the text as simple as possible. Most formatting codes will be removed and replaced on processing the article. The electronic text should be prepared in a way very similar to that of conventional manuscripts (see also the Guide to Publishing with Elsevier: http://www.elsevier.com/guidepublication). See also the section on Electronic artwork.

To avoid unnecessary errors you are strongly advised to use the 'spell-check' and 'grammar-check' functions of your word processor.

\section{Subdivision}

Manuscript files should be structured as follows: (1) Title page, including Disclosure of interest and Acknowledgments, etc.; (2) Manuscript file including Abstract, Keywords, Abbreviations, Main text, References, Legends of figures and tables; (3) Table files; (4) Figure files; (5) Supplementary files; (6) Signed authorship/copyright form; (7) ICMJE forms.

\section{Manuscript Headings}

Original Article level 1 headings are: Methods, Results, Discussion, and Conclusions. Articles should include the level 2 subsection heading Study Limitations at the end of the Discussion section. Longer articles may need other level 2 and/or level 3 subsection headings to clarify their content, especially the Results and Discussion sections. Other types of articles such as Commentaries and Special Communications do not require this format.

\section{Title Page}


Include these elements in the title page in the following sequence, double-spaced: (1) Running head of no more than 40 character spaces (no abbreviations); (2) Title (no abbreviations); (3) Author(s) full name(s) and highest academic degree(s); (4) The name(s) of the institution(s), section(s), division(s), and department(s) where the study was performed and the institutional affiliation(s) of the author(s) at the time of the study. An asterisk after an author's name and a footnote may indicate a change in affiliation; (5) Acknowledgment of any presentation of this material, to whom, when, and where; (6) Acknowledgment of financial support, including grant numbers and any other needed acknowledgments. Explanations of any conflicts of interest; (7) Name, address, business telephone number, and e- mail address of corresponding author; and (8) Clinical trial registration number, if applicable. Please note that clinical trial registration will now be required as of January 1, 2016. The grace period will end January 1, 2017 when registration will be mandatory.

\section{Abstract}

For articles reporting original data (Original Articles, Brief Reports) and Review Articles (including MetaAnalyses), a structured abstract is required (see the Instructions for Structured Abstracts). For other manuscripts (e.g., Commentaries, Editorials and Special Communications), include a conventional, unstructured abstract of no more than 250 words.

\section{Keywords}

All abstracts must include provide 3 to 5 Keywords identified ny the author. Keywords must be selected from the US National Library of Medicine's (NLM) Medical Subject Headings, which is available at http://www.nlm.nih.gov/mesh/MBrowser.html.

\section{Abbreviations}

Archives' editorial policy is to minimize the use of abbreviations. Fewer abbreviations make it easier for the multidisciplinary readership to follow the text. Authors should include a list of abbreviations in their manuscript file directly following the keywords (just above the introduction). Archives uses only standard abbreviations with Davis's and Dorland's as our guides. Abbreviations that are used only in tables, appendices, or figures are not included in the list and should be defined in the table, appendix, or figure legend. However, abbreviations that are in the list need not be re-defined in a table footnote or figure legend. All abbreviation lists must be alphabetized. All abbreviations must be defined upon first mention in the body of the manuscript. The abbreviations SD (standard deviation) and SE (standard error) require no definition in Archives.

\section{Main Manuscript}

\section{Introduction}

State the purpose of the article. Summarize the rationale for the study or observation. Give only pertinent references, and do not review the subject extensively. Do not include data or conclusions from the work being reported. Do not include a heading for this section.

\section{Methods}

Describe the selection of the observational or experimental subjects (patients or experimental animals, including controls) clearly. Discuss eligibility of experimental subjects. Give details about randomization. Describe the methods for any blinding of observations. Identify the methods, equipment and materials, and procedures in sufficient detail to allow others to reproduce the results.Reference established methods, including statistical methods (see below); provide very brief descriptions for methods that have been published but are not well known; describe new or substantially modified methods, give reasons for using them, and evaluate their limitations. Identify precisely all drugs and chemicals used, including generic name(s), dose(s), and route(s) of administration.

While there may be occasional exceptions, Archives is committed to the need for clinical trial reports to be accompanied by adequate periods of follow-up. A lack of sufficient follow-up may be detrimental to a paper's acceptance.

When reporting work with human subjects, indicate whether the procedures followed protocol and accord with the ethical standards of the responsible institutional review board, ethics committee or 
with the Helsinki Declaration of 1975, as revised in 2013, as appropriate for the country where the research took place. ${ }^{2}$

Do not use patients' names, initials, or hospital numbers, especially in any illustrative material. When reporting experiments on animals, indicate whether the procedures followed accord with the institution's committee on animal experimentation or with the National Research Council's guide on the care and use of laboratory animals. Archives may require authors to verify the above procedures.

Describe statistical methods in enough detail to enable knowledgeable readers with access to the original data to verify the reported results. When possible, quantify findings and present them with appropriate indicators of measurement error or uncertainty (eg, confidence intervals [Cls]). Avoid sole reliance on statistical hypothesis testing, such as $P$ values, which fails to convey important quantitative information.

Researchers should report and identify the specific statistical test used and the obtained statistical value. Researchers should supplement the results of any statistical value. Researchers should supplement the results of any statistical significance test with the use of effect size values or Cls. Measures of effect size or Cls should be routinely included in quantitative clinical trials reported in rehabilitation research. The statistical power values and the corresponding type II error probability should always be reported for statistically nonsignificant results.

The investigator should ensure that there is sufficient power to detect, as statistically significant, a clinically meaningful treatment effect of an a priori specified size ${ }^{4}$. References for study design and statistical methods should be to standard works (with pages stated) rather than to papers in which designs or methods were originally reported.

Specify any general use computer programs used. Avoid nontechnical uses of technical terms in statistics, such as "random" (which implies a randomizing device), "normal," "significant," "correlation," or "sample." Define statistical terms, abbreviations, and symbols.

When submitting manuscripts on randomized controlled trials (RCTs), authors must include the CONSORT (Consolidated Standards for Reporting Trials) flow diagram. See the Reporting Guidelines.

\section{Results}

When data are summarized in the Results section, specify the statistical methods used to analyze them. Describe the success of any blinding of observations. Report treatment complications. Give numbers of observations. Report losses to observation (ie, dropouts from a clinical trial). Present results in logical sequence in the text, tables, and illustrations. Archives aims to publish no more than 5 figures per manuscript so restrict tables and figures to those needed to explain arguments and to assess their support. Use graphs as an alternative to tables with many entries; do not duplicate data in graphs and tables. Do not repeat in the text all the data in the tables, illustrations, or both; emphasize or summarize only important observations.

While there may be occasional exceptions, Archives is committed to the need for clinical trial reports to be accompanied by adequate periods of follow-up. A lack of sufficient follow-up may be detrimental to a paper's acceptance.

\section{Discussion}

Emphasize the new and important aspects of the study and the conclusions that follow from them. Do not repeat in detail data or other material given in the introduction or the Results section. Include in the Discussion section the implications of the findings and their limitations, including implications for future research. Authors should address the issue of effect magnitude, in terms of both the statistics reported and the implications of the research. Relate the observations to other relevant studies.

\section{Study Limitations}

Include the subsection (Level 2 heading), "Study Limitations" to discuss the limitations of the study.

\section{Conclusions}

Link the conclusions with the study's goals but avoid unqualified statements not supported by the 
data. Avoid claiming priority and alluding to work that is incomplete. State new hypotheses when warranted, but clearly label them as such. Recommendations, when appropriate, may be included.

\section{Graphical abstract}

Although a graphical abstract is optional, its use is encouraged as it draws more attention to the online article. The graphical abstract should summarize the contents of the article in a concise, pictorial form designed to capture the attention of a wide readership. Graphical abstracts should be submitted as a separate file in the online submission system. Image size: Please provide an image with a minimum of $531 \times 1328$ pixels $(\mathrm{h} \times \mathrm{w})$ or proportionally more. The image should be readable at a size of $5 \times 13 \mathrm{~cm}$ using a regular screen resolution of $96 \mathrm{dpi}$. Preferred file types: TIFF, EPS, PDF or MS Office files. See http://www.elsevier.com/graphicalabstracts for examples.

Authors can make use of Elsevier's Illustration and Enhancement service to ensure the best presentation of their images and in accordance with all technical requirements: Illustration Service.

\section{Highlights}

Highlights are a short collection of bullet points that convey the core findings of the article. Highlights are optional and should be submitted in a separate editable file in the online submission system. Please use 'Highlights' in the file name and include 3 to 5 bullet points (maximum 85 characters, including spaces, per bullet point). See http://www.elsevier.com/highlights for examples.

\section{Acknowledgments}

One or more statements should specify: (1) contributions that do not justify authorship (ie, third-party statistical analysis, writing/editing); and (2) acknowledgments of technical help.

Persons who have contributed intellectually to the manuscript but whose contributions do not justify authorship must be named and their function or contribution described, e.g., "scientific adviser," "critical review of study proposal," "data collection," or "participation in clinical trial." Clerical, administrative, laboratory staff, and participants/subjects in the study should not be acknowledged unless they have contributed significantly to the research, writing, or intellectual quality of the article. Such persons must give permission to be named. Authors are responsible for obtaining written permission from persons acknowledged by name because readers may infer their endorsement of the data and conclusions.

\section{Units}

Metric units are required. Blood pressures in millimeters of mercury $(\mathrm{mmHg})$ and all hematologic and clinical chemistry measurements using the International System of Units (SI).

\section{Footnotes}

Footnotes other than for references are not allowed in the manuscript body.

\section{Artwork}

Preferred file formats are TIFF, EPS, JPEG, and PDF.

$300 \mathrm{dpi}$ is minimum resolution to achieve high quality images. Typical desired resolutions are $300 \mathrm{dpi}$ for black and white and color figures; 500 dpi for combination art (combined photo with line art); and $1000 \mathrm{dpi}$ for line art.

Figures should be numbered consecutively in the order they are first cited in the text. If a figure has been published, acknowledge the original source in the reference list and the figure legend, and submit written permission from the copyright holder to reproduce the material. Permission is required, irrespective of authorship or publisher, except for documents in the public domain.

Letters, numbers, and symbols should be clear and even throughout, and of sufficient size that when reduced for publication each item will still be legible. Titles and detailed explanations belong in the legends for figures, not on the figures themselves. 
Consistency in size within the article is strongly preferred. Any special instructions regarding sizing should be clearly noted. Photomicrographs must have internal scale markers. Symbols, arrows, or letters used in the photomicrographs should contrast with the background.

If photographs of persons are used, either the subjects must not be identifiable or the author must obtain and archive permission to publish the pictures and attest that permission has been granted in the cover letter that accompanies the manuscript submission.

The Editorial Board reserves the right to determine which figures are appropriate for publication. There is no charge for publication of black and white illustrations.

\section{Electronic artwork}

\section{General points}

- Make sure you use uniform lettering and sizing of your original artwork. Preferred fonts: Arial (or Helvetica), Times New Roman (or Times), Symbol, Courier. Number the illustrations according to their sequence in the text. Use a logical naming convention for your artwork files. Indicate per figure if it is a single, 1.5 or 2-column fitting image. For Word submissions only, you may still provide figures and their captions, and tables within a single file at the revision stage. Please note that individual figure files larger than $10 \mathrm{MB}$ must be provided in separate source files.A detailed guide on electronic artwork is available on our website:

http://www.elsevier.com/artworkinstructions.You are urged to visit this site; some excerpts from the detailed information are given here.FormatsRegardless of the application used, when your electronic artwork is finalized, please 'save as' or convert the images to one of the following formats (note the resolution requirements for line drawings, halftones, and line/halftone combinations given below): EPS (or PDF): Vector drawings. Embed the font or save the text as 'graphics'.TIFF (or JPG): Color or grayscale photographs (halftones): always use a minimum of 300 dpi.TIFF (or JPG):

Bitmapped line drawings: use a minimum of 1000 dpi.TIFF (or JPG): Combinations bitmapped line/halftone (color or grayscale): a minimum of $500 \mathrm{dpi}$ is required.Please do not: Supply files that are optimized for screen use (e.g., GIF, BMP, PICT, WPG); the resolution is too low. Supply files that are too low in resolution. Submit graphics that are disproportionately large for the content.

\section{Color artwork}

Color figures (minimum $300 \mathrm{dpi}$ ) will be published without charge when color reproduction is essential to understanding of the material presented.

\section{Figure legends}

A list of figure legends should be provided after the reference list, listing each figure in order by number. Legends/captions should not be embedded in the figure files themselves.

\section{Figure captions}

Ensure that each illustration has a caption. A caption should comprise a brief title (not on the figure itself) and a description of the illustration. Keep text in the illustrations themselves to a minimum but explain all symbols and abbreviations used.

\section{Tables}

Submit each table as a separate file. Accepted file formats are PDF and Word (Please do not upload Excel files). If needed, Excel files will be requested from the authors upon a final editorial decision of accept. Number tables consecutively in the order of their first citation in the text. Include a brief title for each table, include a short or abbreviated heading for each column. Place explanatory matter in footnotes, not in the title or column headings. Explain in footnotes all nonstandard abbreviations that are used in each table. For footnotes, use the following symbols, in this sequence: *, , $\ddagger, \S, \|, \uparrow, \#,{ }^{* *}$, ††, 㧊

Identify statistical measures of variations such as standard deviation and standard error of the mean. Do not use internal horizontal and vertical rules. Be sure that each table is cited in the text in order. Using too many tables in relation to the length of the text may produce typesetting difficulties. 
Data from another published or unpublished source may only be used with permission and must be acknowledged fully. It is the author's responsibility to obtain such permission.

\section{Supplementary data}

Archives accepts electronic supplementary material to support and enhance your scientific research. Supplementary files offer the author additional possibilities to publish supporting applications, highresolution images, background datasets, sound clips, and more. Supplementary files supplied will be published online alongside the electronic version of your article in Elsevier Web products, including ScienceDirect: http://www.sciencedirect.com.

\section{Suppliers}

Before the References section, provide a Suppliers list with contact information (names and complete mailing addresses) for manufacturers of devices and other non-drug products used directly in a study (ie, do not provide such information for products not directly used in your research but mentioned in studies you cite). Identify equipment and/or materials in text, tables, and legends by superscript lower case letters. List suppliers consecutively in the order they are mentioned in the text.

Manufacturer names and locations should not be listed in the text where the product is introduced. Do not list Suppliers in the References list. Do not list drug manufacturers in the Suppliers list.

\section{References}

References in manuscripts accepted by Archives shall include only material that is retrievable through standard literature searches. Number references consecutively in the order in which they first appear in the text. Identify references in text, tables, and legends by superscript Arabic numerals. References cited only in tables or in legends to figures should be numbered in accordance with a sequence established by the first identification in the text of the particular table or figure.

Try to avoid using abstracts as references; "unpublished observations" and "personal communications" may not be used as references, although references to written, not oral, communications may be inserted (in parentheses) in the text. Avoid "personal communication" unless it provides essential information not available from a public source. In this case, cite the name of the person and date of communication in parentheses in the text. For scientific articles, authors should obtain written permission and confirmation of accuracy from the source of personal communication.

Include among the references those papers accepted but not yet published; designate the journal and add "In press." Authors must obtain written permission to cite such papers as well as verification that they have been accepted for publication. Editors will request from the author(s) a copy of the letter from the journal accepting the "in press" article if the manuscript in which it is cited is accepted by Archives. Information from manuscripts submitted but not yet accepted should be cited in the text as "(unpublished observations)" with written permission from the source.

The references must be verified by the author(s) against the original documents. List all authors and/or editors for each reference. Do not insert "et al."

Click here for examples of correct reference formats.

\section{Citations in the running text}

Number references consecutively in the order in which they first appear in the text. Identify references in text, tables, and legends by superscript Arabic numerals. References cited only in tables or in legends to figures should be numbered in accordance with a sequence established by the first identification in the text of the particular table or figure.

\section{Reference management software}

Most Elsevier journals have a standard template available in key reference management packages. This covers packages using the Citation Style Language, such as Mendeley ( http://www.mendeley.com/features/reference-manager) and also others like EndNote http://www.endnote.com/support/enstyles.asp) and Reference Manager ( http://refman.com/downloads/styles). Using plug-ins to word processing packages which are available 
from the above sites, authors only need to select the appropriate journal template when preparing their article and the list of references and citations to these will be formatted according to the journal style as described in this Guide. The process of including templates in these packages is constantly ongoing. If the journal you are looking for does not have a template available yet, please see the list of sample references and citations provided in this Guide to help you format these according to the journal style.

If you manage your research with Mendeley Desktop, you can easily install the reference style for this journal by clicking the link below:

http://open.mendeley.com/use-citation-style/archives-of-physical-medicine-and-rehabilitation

When preparing your manuscript, you will then be able to select this style using the Mendeley plug-ins for Microsoft Word or LibreOffice. For more information about the Citation Style Language, visit http://citationstyles.org.

\section{Reference formatting}

There are no strict requirements on reference formatting at submission. References can be in any style or format as long as the style is consistent. Where applicable, author(s) name(s), journal title/book title, chapter title/article title, year of publication, volume number/book chapter and the pagination must be present. Use of DOI is highly encouraged. The reference style used by the journal will be applied to the accepted article by Elsevier at the proof stage. Note that missing data will be highlighted at proof stage for the author to correct. If you do wish to format the references yourself they should be arranged according to the following examples:

\section{Reference style}

Text: Indicate references by (consecutive) superscript Arabic numerals in the order in which they appear in the text. The numerals are to be used outside periods and commas, inside colons and semicolons. For further detail and examples you are referred to the AMA Manual of Style, A Guide for Authors and Editors, Tenth Edition, ISBN 0-978-0-19-517633-9 (see http://www.amanualofstyle.com). List: Number the references in the list in the order in which they appear in the text. Click here for examples of correct reference formats.

Journal abbreviations in referencesThe titles of journals should be abbreviated according to the style used in MEDLINE. Consult List of Serials Indexed for Online Users,

which is available from the NLM at http://www.nlm.nih.gov/tsd/serials/lsiou.html. Submission checklist

Archives requires the completion and upload of a checklist with each manuscript. Please follow the instructions on the checklist, which can be downloaded here, to ensure all required manuscript elements are included with your submission.

For any further information please visit our customer support site at http://support.elsevier.com.

\section{Use of the Digital Object Identifier}

The Digital Object Identifier (DOI) may be used to cite and link to electronic documents. The DOI consists of a unique alpha-numeric character string which is assigned to a document by the publisher upon the initial electronic publication. The assigned DOI never changes. Therefore, it is an ideal medium for citing a document, particularly 'Articles in press' because they have not yet received their full bibliographic information. Example of a correctly given DOI (in URL format; here an article in the journal Physics Letters B):

http://dx.doi.org/10.1016/j.physletb.2010.09.059

When you use a DOI to create links to documents on the web, the DOls are guaranteed never to change.

\section{Proofs}


Page proofs will be sent from Elsevier by e-mail to the corresponding author (please be sure to notify the Editorial Office [ArchivesMail@archives.acrm.org] of any change to the corresponding author email address provided during the submission process). A link is provided in the e-mail for download of the PDF proof. The PDF proofs can be annotated; for this you will need to download Adobe Reader version 7 (or higher) available free at http://get.adobe.com/reader. The exact system requirements are given at the Adobe site: http://www.adobe.com/products/reader/tech-specs.html. Instructions on how to annotate PDF files will accompany the proofs (also given online). If you are unable to use the PDF annotations function, you may list the corrections (including replies to the Query Form) and return them to Elsevier in an e-mail. Please list your corrections quoting the page and line number from the proof. If, for any reason, this is not possible, then mark the corrections and any other comments (including replies to the Query Form) on a printout of your proof and return by fax, or scan the pages and e-mail. Please use this proof only for checking the typesetting, editing,

completeness and correctness of the text, tables and figures. Significant changes to the article as accepted for publication will only be considered at this stage with permission from the Archives' Editor. We will do everything possible to get your article published quickly and accurately - please return all corrections to Elsevier within 48 hours. It is important to ensure that all corrections are sent back to Elsevier in one communication: please check carefully before replying, as inclusion of any subsequent corrections cannot be guaranteed. Proofreading is solely the author's responsibility. Note that although we make every effort to contact authors, Elsevier may proceed with the publication of your article if no response is received.

\section{AudioSlides}

The journal encourages authors to create an AudioSlides presentation with their published article. AudioSlides are brief, webinar-style presentations that are shown next to the online article on ScienceDirect. This gives authors the opportunity to summarize their research in their own words and to help readers understand what the paper is about. More information and examples are available at

http://www.elsevier.com/audioslides. Authors of this journal will automatically receive an invitation e-mail to create an AudioSlides presentation after acceptance of their paper.

\section{Offprints}

The corresponding author, at no cost, will be provided with a personalized link providing 50 days free access to the final published version of the article on ScienceDirect. This link can also be used for sharing via email and social networks. For an extra charge, paper offprints can be ordered via the offprint order form which is sent once the article is accepted for publication. Both corresponding and co-authors may order offprints at any time via Elsevier's WebShop ( http://webshop.elsevier.com/myarticleservices/offprints). Authors requiring printed copies of multiple articles may use Elsevier WebShop's 'Create Your Own Book' service to collate multiple articles within a single cover ( http://webshop.elsevier.com/myarticleservices/booklets).

You can track your submitted article at http://www.elsevier.com/track-submission. You can track your accepted article at http://www.elsevier.com/trackarticle. You are also welcome to contact Customer Support via http://support.elsevier.com.

\section{References}

1. International Committee of Medical Journal Editors. Recommendations for the Conduct, Reporting, Editing, and Publication of Scholarly Work in Medical Journals. 2013. Available at: http://www.icmje.org. Accessed July June 169, 20140.

2. Committee on Publication Ethics. Flowcharts: Changes in Authorship. nd. Available at: http://www.publicationethics.org/resources/flowcharts. Accessed June 16, 2014.

3. 64th WMA General Assembly. WMA Declaration of Helsinki - Ethical Principles for Medical Research Involving Human Subjects. Available at: http://www.wma.net/en/30publications/10policies/b3/. Accessed June 16, 2014.

4. Ottenbacher KJ. Why rehabilitation research does not work (as well as we think it should). Arch Phys Med Rehabil 1995;76:606-9.

Updated November 5, 2015 
Copyright (C) 2015 Elsevier Inc. All rights reserved. | Privacy Policy | Terms \& Conditions | About Us | Help \& ContactThe content on this site is intended for health professionals.Advertisements on this site do not constitute a guarantee or endorsement by the journal, Association, or publisher of the quality or value of such product or of the claims made for it by its manufacturer.

\section{QUALIS}

\section{Periódicos}

\begin{tabular}{|l|l|l|c|}
\hline \multicolumn{2}{|l}{ TŚtulo } & \multicolumn{1}{c|}{ Área de Avaliação } & Classificação \\
\hline $0003-9993$ & $\begin{array}{l}\text { Archives of Physical Medicine } \\
\text { and Rehabilitation (Print) }\end{array}$ & BIOTECNOLOGIA & B1 \\
\hline $0003-9993$ & $\begin{array}{l}\text { Archives of Physical Medicine } \\
\text { and Rehabilitation (Print) }\end{array}$ & EDUCAÇÃO FÍSICA & A1 \\
\hline $0003-9993$ & $\begin{array}{l}\text { Archives of Physical Medicine } \\
\text { and Rehabilitation (Print) }\end{array}$ & ENGENHARIAS IV & B1 \\
\hline $0003-9993$ & $\begin{array}{l}\text { Archives of Physical Medicine } \\
\text { and Rehabilitation (Print) }\end{array}$ & MATERIAIS & B2 \\
\hline $0003-9993$ & $\begin{array}{l}\text { Archives of Physical Medicine } \\
\text { and Rehabilitation (Print) }\end{array}$ & MEDICINA II & B1 \\
\hline $0003-9993$ & $\begin{array}{l}\text { Archives of Physical Medicine } \\
\text { and Rehabilitation (Print) }\end{array}$ & NUTRIÇÃO & B1 \\
\hline $0003-9993$ & $\begin{array}{l}\text { Archives of Physical Medicine } \\
\text { and Rehabilitation (Print) }\end{array}$ & PSICOLOGIA & A2 \\
\hline
\end{tabular}




\section{ANEXO 7 - NORMAS DA REVISTA E QUALIS - MANUSCRITO 3}

Journal of Manipulative and Physiological Therapeutics - fator de impacto

JEURNAL DF MANIPULATIVE AND PHYSIDLEGIEAL THERAPEUTIES

Mobile

ه

RSS FeedsLogin | Register | Subscribe

Articles \& Issues Collections For Authors Journal Info Society Info Subscribe Help More Periodicals

All Content Search Advanced Search

\section{General information}

The Journal of Manipulative and Physiological Therapeutics (JMPT) is an international peer-reviewed journal dedicated to the advancement of the science of manipulative and physiological therapeutics and chiropractic health care principles and practice. Submissions must be original work, not previously published, and not currently under consideration for publication in another medium, including both paper and electronic formats. The JMPT does not publish articles containing material that has been reported at length elsewhere. The journal follows the standards as set forth in the Uniform Requirements for Manuscripts (www.icmje.org).

\section{JMPT MANUSCRIPT FORMS}

Title Page Form CopyrightConflict of Interest Case/Case Series

\section{MANUSCRIPT CATEGORIES}

Manuscripts should fit into one of the following categories (text word limit does not include abstract, tables, or reference word count):

\section{Experimental and observational investigations}

Reports of new research findings. These studies may include investigations into the improvement of health factors, causal aspects of disease, and the establishment of clinical efficacies of related diagnostic and therapeutic procedures. These types of studies may include: clinical trials, intervention studies, cohort studies, case-control studies, observational studies, cost-effectiveness analyses, epidemiologic evaluations, studies of diagnostic tests, etc. These reports should follow current and relevant guidelines (eg, CONSORT, MOOSE, QUOROM, STARD, TREND, etc.) (text word limit, approximately 4000 words)

\section{Systematic reviews and meta-analyses}


Assessments of current knowledge of a particular subject of interest that synthesize evidence relevant to welldefined questions about diagnosis, prognosis, or therapy with emphasis on better correlation, the demonstration of ambiguities, and the delineation of areas that may constitute hypotheses for further study. (text word limit, approximately 4000 words)

\section{Clinical guidelines}

Succinct, informative, summaries of official or consensus positions on issues related to health care delivery, clinical practice, or public policy. (text word limit, approximately 2000 words)

\section{Technical reports}

Reporting and evaluation of new or improved equipment, procedures, or the critical evaluation of old equipment or procedures that have not previously been critically evaluated. (text word limit, approximately 2000 words)

\section{Case series}

Case series are retrospective descriptions of the diagnosis and treatment of several cases of a similar condition. (text word limit, approximately 1500 words)

\section{Letters to the editor}

Communications that are directed specifically to the editor that add to the information base or clarify a deficiency in paper recently published in the JMPT (must be within the last 2 months) and include relevant references to substantiate comments. No unidentified letters are accepted for publication. All letters are subject to editing and abridgement. If a letter is accepted for publication, a blinded copy will be sent to the author of the article who will have an opportunity to provide a response and new information that will be considered for publication along with the letter. Direct communication between the writer of a letter and the author of an article should be avoided, because in the interest of scientific objectivity differences of opinion are best handled by a third party - the editor - who can serve as an arbitrator if there is a dispute, thus avoiding unnecessary irritations to either party. Also, if deficiencies exist in an article published in the JMPT, all readers (and the scientific community in general) have a right to be informed. For more information about letters to the editor, please read this editorial. (text word limit, 500 words maximum, reference limit 8).

\section{EDITORIAL POLICIES}

\section{Authorship}

All authors of papers submitted to JMPT must have an intellectual stake in the material presented for publication and must be able to answer for the content of the entire work. Authors must be able to certify participation in the work, vouch for its validity, acknowledge reviewing and approving the final version of the paper, acknowledge that the work has not been previously published elsewhere, and be able to produce raw data if requested by the editor. All authors are required to complete and submit an authorship copyright form.

As stated in the Uniform Requirements (www.icmje.org), credit for authorship requires all three of the following: 1) substantial contributions to conception and design, or acquisition of data, or analysis and interpretation of data; 2) drafting the article or revising it critically for important intellectual content; and 3) final approval of the version to be published. Authors should meet conditions 1, 2, and 3. Each author must sign a statement attesting that he or she fulfills the authorship criteria of the Uniform Requirements and is included on the copyright assignment form. Authors are required to designate their level of participation of authorship on the

authorship form. A change in authorship after submission must be signed by all authors and submitted to the editor prior to being considered.

\section{Contributorship}

For each author, how the author contributed to the manuscript should be included in the title page form. For example: concept development (provided idea for the research), design (planned the methods to generate the results), supervision (provided oversight, responsible for organization and implementation, writing of the manuscript), data collection/processing (responsible for experiments, 
patient management, organization, or reporting data), analysis/interpretation (responsible for statistical analysis, evaluation, and presentation of the results), literature search (performed the literature search), writing (responsible for writing a substantive part of the manuscript), critical review (revised manuscript for intellectual content, this does not relate to spelling and grammar checking), and other (list other specific novel contributions).

\section{Human subjects and animal studies}

Studies with human subjects or animals must go through approval from the appropriate ethics review board/committee, animal board, or IRB in advance. The JMPT endorses the ICMJE guidelines and the Declaration of Helsinki. All related conditions regarding the experimental use of human subjects and their informed consent apply. Studies using animals should follow the Animal Research: Reporting In Vivo Experiments (ARRIVE) guidelines. Information about review board approval should be included the Methods section of the paper. Manuscripts that report the results of experimental investigations with human subjects must include a statement that informed consent was obtained (in writing, from the subject or legal guardian) after the procedure(s) had been fully explained.

Evidence of board approval should be submitted at the initial time of submission. When applicable, a signed letter from the HIPAA compliance officer should be submitted. Fax (630) 839-1792 or email cjohnson@nuhs.edu.

\section{Clinical trial registration}

Clinical trials should be included in a clinical trial registry. The clinical trial registration number should be included in the methods section of the manuscript. Clinical trials should be registered in a public trials registry at or before the onset of patient enrollment as a condition of consideration for publication. This policy applies to clinical trials starting enrollment after July 1, 2005. For trials that began enrollment before this date, registration should be completed by September 13, 2005, before considering the trial for publication. The ICMJE (www.icmje.org) defines a clinical trial as a study that prospectively assigns human subjects to intervention or comparison groups to evaluate the cause-andeffect relationship between an intervention and a health outcome. Trial registration numbers and the URLs for the registry should be included in the title page form at the time of submission.

\section{Patient anonymity}

It is the authors' responsibility to maintain appropriate records as well as protect patients' identity. Ethical and legal considerations require careful attention to the protection of the patient's anonymity in case reports and other publications. Identifying information such as names, initials, actual case numbers, and specific dates must be avoided; identifying information about a patient's personal history and characteristics should be disguised. Anonymity should be maintained for case reports regardless of the patient providing permission to publish. Photographs or artistic likenesses of subjects, patients, or models are publishable only with their written consent or the consent of legal guardian; the signed consent form, giving any special conditions, must accompany manuscript.

\section{Case consent form}

Case series must be accompanied by completed and signed patient consent to publish form from each patient. The case consent forms should be uploaded at the time of initial manuscript submission. Authors should include a statement in the text, without divulging personal identifiers, that the patient(s) gave consent to have personal health information published.

\section{HIPAA compliance}

For more information about HIPAA as it relates to obtaining patient consent for publication, please refer to http://privacyruleandresearch.nih.gov/faq.asp or your country's legal guidelines.

\section{Conflict of interest}

Authors - Each author is required to complete a conflict of interest form (created by the ICMJE) and submit this form at the time of initial submission. Conflict of interest exists when an author has financial or other interests that may influence his or her actions in regard to the authors' work, manuscript development, or decisions. Conflicts of interest that exist, or that are perceived to exist, for individual authors in connection with the content of the paper shall be disclosed to the JMPT at the time of 
submission. In addition to the form, any concerns or additional conflict of interest issues may be included in the cover letter to the editor. Authors must also disclose to the editor in the cover letter the conflicts of interest of any other person or entity involved with the paper (eg, non-author, contributor, funding body) In recognition that it may be difficult to judge material from authors where conflict of interests are concerned, authors should be ready to answer requests from the editor regarding potential conflicts of interest. The editor makes the final determination concerning the extent of information included in the published paper.

It is expected that authors are truthful when declaring conflicts on their submission materials. An editor's role is not to be policeman, so the burden is upon the author to properly declare COI. If an author did not accurately and completely declare their interests upon submission, and it is discovered later, the editor will follow up with an ethics investigation. The results may include rejection or retraction of the paper, prevention of future submissions, and notification of ethical misconduct to the proper authorities.

Editorial staff and Peer Reviewers - It is expected that people involved with handling manuscripts for the journal will properly disclose their financial and professional interests that may be be viewed as potential conflicts of interest and recuse themselves from any actions in which their conflicts of interest will hamper their judgment or actions. Peer reviewers should inform the editor if they feel they are not able to properly peer review a manuscript and recuse themselves from reviewing that manuscript. Editorial staff should disclose information that readers may perceive might influence decisions in journal editing. Disclosure statements about potential conflicts of interest for the journal staff should be published regularly. Please refer to ICMJE website for more information on COI.

\section{Funding sources}

Sources of financial support of the study, such as grants, funding sources, equipment, and supplies, should be clearly stated in the title page form. The role of funding organizations, if any, in the conduct of the study should be described in the Methods section of the manuscript. If the study is funded directly by an NIH grant or other national funding, it is the corresponding author's responsibility to inform the editor and mark this information on the copyright form at the time of submission.

\section{Copyright of journal contents}

Materials published in the JMPT are covered by copyright. No content published by the JMPT (either in print or electronic) may be stored or presented in other locations such as on another private website, an organization's site, or displayed or reproduced by any other means, without the express permission of the copyright holder.

\section{Redundant or duplicate publication}

Manuscripts must be submitted to only one journal at a time and published in only one journal. The JMPT does not publish articles containing material that has been reported at length elsewhere. The corresponding author must include in the cover letter a statement to the editor about all submissions and previous materials that might be considered to be redundant or duplicate publication of similar work, including if the manuscript includes materials on which the authors have published a previous report or have submitted similar or related work to another publication. Copies of the related material may be requested by the editor in order to assist with the editorial decision of the paper.

If redundant or duplicate publication is attempted or occurs without proper disclosure to the editor, editorial action will be taken as follows. The results may include rejection or retraction of the paper, prevention of future submissions, and notification of ethical misconduct to the proper authorities. If it is confirmed that a paper is a duplicate or redundant publication and is discovered in the prepublication phase, the paper will be rejected, even if an accept notice has been distributed previously to the authors. If duplicate or redundant publication is confirmed after publication, the paper will be retracted and the appropriate boards/institutions notified.

\section{Non-compliance with author instructions}

Authors who do not comply with the items set forth in these instructions may have the submission returned, rejected, or brought to higher authorities, such as ethics, licensing, or institutional boards for further review at the editor's discretion. 


\section{EDITORIAL PROCESS}

\section{Pre-peer review, and internal review by editors}

To insure that only relevant and appropriate papers are sent to peer review, submitted manuscripts are pre-reviewed for relevance, appropriate submission format, and basic quality before sending out to peer review. Reasons for early rejection may include: the submission does not meet the requirements as stated in the instructions for authors, the work is of poor quality, and/or the topic is not relevant to the mission of journal.

The editorial staff reads each manuscript and then decides whether to send the paper to outside reviewers. If a submission is rejected without external review, the author will typically be notified electronically within 2 to 3 weeks of receipt. Over $80 \%$ of submitted papers are sent to external peer review, which is usually made up of at least 2 reviewers, but may be more.

\section{Review process}

The JMPT uses double-blind peer review methods (author and reviewer are blinded). The journal staff will do their best to support blinded review methods, however due to the special nature of the topics published, we cannot guarantee that reviewers or authors may be able to guess the identity of each other.

All manuscripts are subject to blind (without author or institutional identification) critical review by experts in the related field to assist the editor in determining appropriateness to JMPT objectives, originality, validity, importance of content, substantiation of conclusions, and possible need for improvement. Manuscripts are considered privileged communications and should not be retained or duplicated during the review process. Reviewers' comments may be returned with the manuscript if rejected or if strong recommendations for improvement are made. All reviewers remain anonymous.

\section{Rapid review}

Rapid review speeds up the process of peer review and publication. Priority will be given to large clinical trials and meta-analysis. Only manuscripts that are of very high quality that have findings likely to directly influence clinical practice immediately will be considered.

Authors who feel that their research warrants rapid review should email the editor and submit justification regarding the merits of the paper to substantiate its inclusion for rapid review. The editor will make the final decision regarding the suitability of a submission for rapid review and publication. If a paper is not deemed appropriate by the editor for rapid review, the manuscript may still be submitted through the regular submission process and timeline.

If a manuscript is accepted for rapid review, it will then be handled through an expedited peer review process for decision. The results may include acceptance, major revision, minor revision, or rejection. Inclusion in the rapid review process guarantees neither acceptance of the paper nor promise of rapid publication if accepted. Each decision and paper review will be done separately. All papers that are selected for rapid review will be processed through peer review.

The expedited review process will take approximately 15 business days. Authors will be notified about revision no later than 5 weeks after the manuscript is initially received. If revision is requested, authors of a rapid review submission should return a revised manuscript within 2 weeks of notification. At this time, a decision will be made for acceptance or rejection. If the manuscript is accepted, it will be scheduled immediately for in press publication.

\section{Criteria for editorial decisions}

The JMPT can publish only a portion of all papers submitted each year. Papers are selected based on quality and strength of the paper in regard to scientific merit and the potential impact on improving patient care.

\section{Revisions, rejections, and resubmissions}

Processing of a manuscript for peer review does not imply acceptance to publish, even though the paper may be found to be within JMPT editorial objectives. Submissions may receive one of five 
responses from the editor: 1) incomplete or not ready for submission, 2) major revision, 3) minor revision, 4) accept, or 5) reject. Aside from rejection for uncorrectable faults, a well-compiled manuscript may also be rejected because it adds little new information to work that was previously published in the literature or addresses a new topic that deserves more in-depth reporting. In these cases, the editor may provide the author of a rejected manuscript recommendations that may be helpful for submission elsewhere.

If the authors have been given the opportunity by the editor to make specific changes to a manuscript and return it for further consideration, this is considered a "revision." The manuscript will have the same manuscript number and may be sent out to the same or different reviewers, depending on the needs of the revision. A request for revision does not imply that the manuscript will be accepted. Manuscripts that are revised and returned may still be rejected.

If the authors have received a rejection decision but wish the editor to reconsider the decision, this is considered a "resubmission." A new file will be created, and the paper will receive a new manuscript number. The cover letter must explain that the paper is being resubmitted and substantiated with explanations for why the paper should be allowed to be resubmitted.

\section{Acceptance for publication}

Once a manuscript has been accepted, the authors should not distribute content relating to the article while it is being prepared for publication. It is permissible at this time to refer to this manuscript as "accepted for publication" in a forthcoming issue of JMPT; however, it is requested that no further details of the paper, or the research on which it may have been based, be given out in consideration that abridged or inexact versions of research or scholarly work can be misleading, or even hazardous where clinical procedures are involved.

Authors may use Elsevier's Author Gateway (http://authors.elsevier.com ) to track accepted articles and set up e-mail alerts to inform you of when an article's status has changed. Answers to questions arising after acceptance of an article, especially those relating to proofs, are provided after registration of an article for publication.

Accepted papers will be edited for clarity, journal style, and accuracy of information. The intention is to provide the highest quality version of the paper for final publication. Authors will have the opportunity to review the manuscript before final publication during the proof stage to make sure all corrections are accurate. The editor reserves the right to accept or deny any correction requests from authors prior to final publication.

\section{Proofs}

All manuscripts accepted for publication are subject to post acceptance editing; revision may be necessary to ensure clarity, completeness, conciseness, correct usage, and conformance to approved style. Almost all papers that are accepted require some editorial revision before publication. Authors will have the opportunity to review corrections/revisions made during the copy editing process during the reviewing of the proofs. Editors will work with authors to arrive at agreement when authors do not find the revisions acceptable, but the JMPT reserves the right to refrain from publishing a manuscript if discussion with the author fails to reach a solution that satisfies the editors. The journal reserves the right to deny requested changes that do not affect accuracy. Authors may be charged for changes to the proofs beyond those required to correct errors or to answer queries. Authors must carefully check and correct the proofs and reply within 24 to 48 hours of receipt and follow all instructions in the proof email.

\section{Publication scheduling of accepted papers and proofs}

Authors will be sent proofs by email. Authors who cannot examine email proofs by the deadline (48 hours of receipt) should email the editor to designate a colleague who will review proofs. All requests for changes within the proofs are reviewed and either approved or denied by the editor. Authors should email promptly for additional information requests from the journal personnel. Once proof changes have been submitted and approved by the editor, no further changes will be considered.

JMPT e-papers Starting with the January 2002 issue, the JMPT initiated an electronic paper section in the journal. Electronic papers have their abstract published in the print version of the journal, while the full-text version of the paper is included on the JMPT web site (www.jmptonline.org). While the editor 
will attempt to honor requests to publish or not publish a paper as an E-paper, the editor reserves the right to make a final decision as to whether a given paper will be published as an E-paper. It is important to note that electronic publication includes all the same rights and privileges as print publication, including inclusion in indexing agency databases.

\section{Funding sources and NIH funded studies}

Statements about funding sources and conflicts of interests should be included in the title page form. If there were no funding sources or identified conflicts of interest to declare, then this should be clearly stated.

The JMPT is compliant with the open access NIH publication policy and will deposit the final version of the published paper to PubMedCentral (PMC) within 12 months of final publication. It is the corresponding author's responsibility to inform the editor in both the cover letter and the copyright form that the study was directly funded by an NIH grant.

\section{Reprints and copies}

Authors of papers published in the JMPT are encouraged to make reprints available to interested members of the scientific, academic, and clinical communities so that the inherent knowledge may be more widely disseminated; a reprint order form will be provided with the proofs to facilitate ordering quantity reprints. One complimentary copy of the JMPT issue in which an author's work appears will be provided at no charge to the corresponding author. Additional copies, if desired, must be ordered at regular cost directly from the publisher. Authors are responsible for payment of reprints or additional copies.

\section{Reproductions}

The entire content of the JMPT is protected by copyright, and no part may be reproduced (outside of the fair use stipulation of Public Law 94-553) by any means without prior permission from the editor or publisher in writing. In particular, this policy applies to the reprinting of an original article in print or in electronic format, in another publication and the use of any illustrations or text to create a new work.

\section{Sponsored Access}

For those authors who wish to make their article open access, the JMPT offers authors the option to sponsor non-subscriber access to individual articles. The charge for article sponsorship is $\$ 3,000$. This charge is necessary to offset publishing costs - from managing article submission and peer review, to typesetting, tagging and indexing of articles, hosting articles on dedicated servers, supporting sales and marketing costs to ensure global dissemination via ScienceDirect, and permanently preserving the published journal article. The fee excludes taxes and other potential author fees such as color charges which are additional.

Authors may select this option after receiving notification that their article has been accepted for publication. This prevents a potential conflict of interest where a journal would have a financial incentive to accept an article.

Authors who have had their article accepted and who wish to sponsor their article to make it available to non-subscribers should complete and submit the order form. Note, the fee is waived with $\mathrm{NIH}$ funded articles.

\section{SUBMISSION INFORMATION}

\section{Manuscript preparation and submission}

All manuscripts must be submitted through the Journal of Manipulative and Physiological Therapeutics online submission and review Web site ( http://ees.elsevier.com/jmpt). Authors may send queries concerning the submission process, manuscript status, or journal procedures to the Editorial Office at cjohnson@nuhs.edu.

Once you have uploaded your submission files, the system automatically generates an electronic (PDF) proof for your review. All correspondence, including the Editor's decision and request for revisions, will be sent by e-mail to the corresponding author. 
Revised manuscripts should be accompanied by an additional Word file with responses to all editor requests and reviewers' comments. This file should contain an itemized list addressing each of the revision requests and demonstrate how these have been addressed in the manuscript. The preferred order of files is as follows: cover letter, response to reviews (revised manuscripts only), manuscript file(s), figure(s).

Authors who are unable to provide an electronic version or have other circumstances that prevent online submission must contact the Editorial Office prior to submission to discuss alternate options. The Publisher and Editors regret that they are not able to consider submissions that do not follow these procedures.

\section{Materials due at initial submission}

All materials associated with the manuscript are due at the time of initial submission. These include: cover letter, title page form, manuscript files, assignment of copyright forms for all authors, conflict of interest forms for all authors, and any permission forms (eg, patient consent to publish forms, permission to have name printed in acknowledgements, permission to reprint table or figure, permission to include person's picture, etc.). It is the corresponding author's responsibility to obtain these permissions and upload them to the website. In the event that the paper is rejected, the permissions and files associated with this manuscript will no longer be valid so that the authors may pursue publication elsewhere.

\section{File requirements}

Original source files, not PDF files, are required for submission. Files should be labeled with appropriate and descriptive file names (eg, SmithText.doc or Fig1.tif).

\section{Revision}

Manuscript revisions are expected within 30 days of request for revision. The corresponding author should contact the editor if there are any questions or more time is needed.

\section{Materials due during revision}

If revision has been requested, all comments, concerns, suggestions must be addressed and include whether the change is made or not. The corresponding author should upload a Word document with a list of itemized changes made in the manuscript addressing each of the revision requirements.

Changes made in the manuscript (insertions or corrected information) should be highlighted the within the text (either highlight or color font) to show reviewers and editor where the changes have been made.

\section{SUBMISSION COMPONENTS AND REQUIREMENTS}

Submission check listThe following items should be ready before submitting to the JMPT website:

1. Coverletter2. Titlepageform3.

BlindedmanuscriptWordfile(doesnotincludeauthornameorotheridentifyinginformation):

structured abstract body of manuscript referencestables

4. Figures(separateJPEGfilesnobiggerthan2MB)5. Signedassignmentofcopyrightformsforeachauthor6. Completedconflictofinterestformforeachauthor7.

Permissionstopublish,consentforms, permissionsforms, forhumanoranimalstudies, evidenceofboardappr oval.

\section{Cover letter}

The cover letter should explain why the paper should be published in the JMPT rather than elsewhere and if the submission is original and not currently under consideration for publication in another peerreviewed medium. The cover letter should include a statement of intent to submit to the JMPT. The cover letter may also include any special information regarding the submission that may be helpful in its consideration for publication. Authors may recommend reviewers for consideration and should 
include name and email of the suggested reviewers. If the study was funded by an NIH grant, this information should be included in the cover letter.

\section{Title page}

Please fill in title page form from the JMPT submission website.

\section{Blinded manuscript file}

\section{Manuscript format and style}

Manuscripts must be prepared in accordance with the Declaration of Vancouver "Uniform Requirements for Manuscripts Submitted to Biomedical Journals" (available from the JMPT Editorial Office or from www.icmje.org). The manuscript should be in double-spaced format. Do not break any words (hyphenate) at the end of any line and do not insert hard page breaks. The journal follows American Medical Association Manual of Style (10th ed. Oxford University Press, NY, 2007).

\section{Structured abstract}

The structured abstract should be no more than 250 words. The abstract should consist of 4 paragraphs, labeled: Objectives, Methods (include relevant information such as design, subjects/population, setting, statistical methods, etc), Results, and Conclusions.

\section{Manuscript organization}

The text of observational and experimental articles is usually divided into sections with the headings Introduction, Methods, Results, and Discussion. Longer articles may need subheadings within some sections to clarify or break up content. Other types of articles such as case reports, reviews, editorials, and commentaries may need other formats. Studies with designed that have guidelines should follow published guidelines. (eg, CONSORT, MOOSE, QUOROM, STARD, TREND, etc.) Any questions about format should be directed to the editor.

\section{Introduction}

Clearly state the purpose of the article. Summarize the rationale for the study or observation. Give only pertinent references and do not review the subject extensively; the introduction should serve only to introduce what was done and why it was done. State the specific purpose, research objective, or hypothesis tested by the study (typically found at the end of the introduction section).

\section{Methods}

The selection and description of participants, technical information, and statistics used should be reported in this section. Describe the selection of the observational or experimental subjects (patients or experimental animals, including controls). Papers of a specific study design should follow current and relevant guidelines (e.g., CONSORT, MOOSE, QUOROM, STARD, TREND, etc.) and include appropriate materials in the text. Identify the methods, apparatus (manufacturer's name and address in parentheses) and procedures in sufficient detail to allow others to reproduce the work for comparison of results. Give references to establish methods, provide references and brief descriptions for methods that have been published but may not be well known, describe new or substantially modified methods and give reasons for using them and evaluate their limitations.

When reporting experiments with human subjects, indicate the procedures used in accordance with the ethical standards of the Committee on Human Experimentation of the institution in which the research was conducted and/or were done in accordance with the Helsinki Declaration of 1975. Clearly indicate the ethics review board or IRB that approved the study. When reporting experiments on animals, indicate whether the institution's or the National Research Council's guide for the care and use of laboratory animals was followed. Do not use patient names, initials, or hospital numbers or in any manner give information by which the individuals can be identified. The author may be requested to provide the editor documentation from the ethics board and methods used to review the work.

The source(s) of support in the form of funds, grants, equipment, or other real goods should be clearly stated in the Methods section. 


\section{Statistics}

Describe the statistical methods in enough detail that would allow a knowledgeable reader with access to the original data to verify the results. Findings should include appropriate indicators of measurement error or uncertainty, such as confidence intervals.

Examples of statistical details that should be included in the methods section are: the eligibility of experimental subjects, details about randomization, methods for blinding, complications of treatment, numbers of observations, dropouts from a clinical trial, the statistical programs used. In the results section, state the statistical methods used to analyze the results. All statistical terms, abbreviations, and symbols should be defined.

Include numbers of observations and the statistical significance of the findings when appropriate. Detailed statistical analyses, mathematical derivations, and the like may sometimes be suitably presented in the form of one or more appendixes.

\section{Results}

Present your results in logical sequence within the text, tables, and figures. Do not repeat findings in multiple places (eg, do not include the same data in both text and tables). Emphasize or summarize only important observations, do not discuss findings in this section.

\section{Discussion}

The discussion should emphasize the important aspects of the study and include conclusions that follow from these observations. Do not repeat data presented in the Results section and do not include information or work that is not directly relevant to the study. State new hypotheses when indicated, but clearly label them as such. Statements that are unsupported, that generalize, or that over extrapolate the findings should not be included.

Conclusions that may be drawn from the study may be included in the discussion; however, they may be more appropriately presented in a separate section. The principal conclusions should be directly linked to the goals of the study. Unqualified statements and conclusions not supported by your data should not be included. Avoid claiming priority or referring to work that has not been completed or published. State new hypotheses when warranted but clearly label them as such. Recommendations (for further study, etc), when appropriate, may be included.

\section{Limitations subsection}

Place limitation subsection at the end of the Discussion section. List and discuss the limitations of the study, possible sources of bias, and any reasonable alternate explanations for the findings and interpretation for the study.

\section{Acknowledgments}

Acknowledge only those who have made substantive contributions to the study itself; this includes support personnel such as statistical or manuscript review consultants, but not subjects used in the study or clerical staff. Clearly state what each contributor has provided. Authors are responsible for obtaining the written permission (to be included at time of submission) that is required from persons, institutions, or businesses being acknowledged by name because readers may infer their endorsement of the data and conclusions.

\section{References}

Authors are responsible for accurate reference and citation information, especially accuracy of author names, journal titles, volume numbers, and page numbers. References should be numbered consecutively when they are first used in the text. Reference citation in the text should be in superscript format and after punctuation (eg, The quick fox jumped over the dog. ${ }^{1}$ ). References should be listed in numeric order (not alphabetically) following the text pages. The original citation number assigned to a reference should be reused each time the reference is cited in the text, regardless of its previous position in the text: do not assign it another number. References should not be included in abstracts. References that are only used in tables or figure legends should be numbered in the sequence established by the first use of the particular table or figure in the text. 
Only references that provide support for a particular statement in the text, tables, and/or figures should be used. Reference or referring to unpublished work should be avoided. Excessive use of references should be avoided. Each reference should only be listed in the reference section once.

Authors are responsible to verify references against the original document and not from reading the abstract alone. Care should be taken to accurately represent the original work and not misconstrue the original meaning of the paper.

\section{Unacceptable reference sources}

Using only the abstract, referring to "unpublished observations" and "personal communications" should be avoided. Unpublished references (submitted but not accepted) should not be listed as references. Manuscripts that are accepted but not yet published may be included in the references with the designation "in press." The author should obtain written permission to cite these papers and may be requested by the editor to provide documentation to verify the paper was accepted for publication. For the most part, sources of information and reference support for a bioscientific paper should be limited to journals (rather than books) because that knowledge is generally considered more recent and (in the case of refereed journals) more accurate.

\section{Reference style}

Reference style should be in accordance with that specified by the US National Library of Medicine. Specific examples of correct reference form for journal articles and other publications can be found at http://www.nlm.nih.gov/bsd/uniform_requirements.html.

The format for at typical journal article is as follows:

1. Lastnameofauthor(s)andtheirinitialsincapitalsseparatedbyaspacewithacommaseparatingeachauthor.( Listallauthors when 6 or fewer; when 7 or more, list only the first 6 and add et al.)

2. Titleofarticlewithfirstwordcapitalizedandallotherwordsinlowercase,exceptnamesofpersons, places, etc

3. Nameofjournal,abbreviatedaccordingtolndexMedicus http://www.nlm.nih.gov/tsd/serials/lji.html;yearofpublication (followed by a semicolon); volume number (followed by a colon); and inclusive pages of article (with redundant number dropped, ie, 105-10).

\section{Tables}

Tables should be placed at the end of the blinded manuscript file at the time of submission. If the paper is accepted, tables will be placed appropriately in the final publication. Tables should be numbered as they appear in the text (e.g., Table 1). Identify statistical measures of variation, such as standard deviation and standard error of mean. If data are used from another source, the author should acknowledge the original source in the text and include the written permission from the copyright holder to reproduce the material with the submission.

Using Arabic numerals, number each table consecutively (in the order in which they were listed in the text in parentheses) and supply a brief title to appear at the top of the table above a horizontal line; place any necessary explanatory matter in footnotes at the bottom of the table below a horizontal line

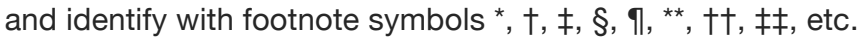

Do not submit tables as photographs. Avoid the use of too many tables in relation to length of the text, as this may produce difficulties in layout of the pages. Avoid the use of tables that do not fit in the 'portrait' layout. Table contents and number of tables may be subject to editing.

Type legends for tables above each table. Include expanded version of all acronyms and symbol meanings in the legend. Identify each legend with Arabic numerals in the same manner and sequence as they were indicated in the text in parentheses (eg, Table 1). Include in the manuscript text where the table should be placed. For example "call out" where the table should be located using (Table 1) in the text.

\section{Terminology}


Standard spelling and terminology should be used whenever possible. Avoid creating new terms or acronyms for entities that already exist. Technical terms that are used in statistics should not be used as non-technical terms, such as "random" (which implies a randomizing device), "normal," "significant" (which implies statistical significance), and "sample."

\section{Units of Measurement}

In most countries the International System of Units $(\mathrm{SI})$ is standard, or is becoming so, and bioscientific journals in general are in the process of requiring the reporting of data in these metric units. However, insofar as this practice is not yet universal, particularly in the United States, it is permissible for the time being to report data in the units in which calculations were originally made, followed by the opposite unit equivalents in parentheses; ie, English units (SI units) or SI units (English units). Nevertheless, researchers and authors considering submission of manuscripts to the JMPT should begin to adopt SI as their primary system of measurement as quickly as it is feasible.

\section{Abbreviations and symbols}

Use only standard abbreviations for units of measurement, statistical terms, biological references, journal names, etc. Avoid abbreviations in titles and abstracts. The full term should precede its abbreviation for the first use in the manuscript, unless it is a standard unit of measurement. For standard abbreviations, consult the following: 1) Uniform requirements for manuscripts submitted to biomedical journals (Ann Intern Med 1997;126:36-47); 2) American Medical Association manual of style. 10th ed. Baltimore: Williams \& Wilkins; 2007; 3) Scientific style and format, the CBE manual for authors, editors, and publishers. 6th ed. Cambridge (UK): Cambridge University Press; 1994.

\section{Figures}

Figures include images, charts, graphs, and lists of information (eg, inclusion criteria). Figures should not be included in the manuscript file. Instead, they should be uploaded separately. Illustrations (including lettering, numbering and/or symbols) must be of professional quality and of sufficient size so that when reduced for publication all details will be clearly readable. Rough sketches with freehand or typed lettering are not acceptable.

Include legends for figures after the reference section in the blinded manuscript file. Identify each figure with Arabic numerals in the same sequence as they appear in the text in parentheses (eg, Fig 1). Do not type legends in the image file. When symbols, arrows, numbers, or letters are used to identify parts of the illustrations, identify and explain each one clearly in the legend. Include in the manuscript text where the figure should be placed. For example "call out" where the figure should be located using (Figure 1) in the text.

Color versions of all figures are preferred. Hard copy will be printed in black and white and electronic version will include color at no extra cost to the author. All illustrations (including radiographs, diagnostic imaging) must be uploaded as at least 200 dpi resolution in JPEG format. The file should be $2 \mathrm{MB}$ or less in size. Figures should be submitted as separate JPEG files and not embedded in the manuscript or Word file. Each figure should be saved using the figure number in its file name (eg, Fig1) and uploaded as a separate file. Original data (eg, Excel file) for graphs or charts may be requested by the editor if the submitted figure is not clear or of poor quality for printing. Typically no more than eight figures are acceptable (eg, Fig 1A and Fig 1B are considered two figures).

If photographs of persons are used the submission must be accompanied by signed written permission to publish the photographs. If a figure has been previously published, acknowledge the original source and submit written permission from the copyright holder to reproduce the image. Permission is required, regardless of authorship or publisher, except for documents in the public domain, in this case the source of the image should be clearly labeled. Since JMPT articles appear in both the print and online versions of the journal, and wording of the letter should specify permission in all forms and media. Failure of the author to obtain electronic permission rights will result in the images not appearing in the paper or rejection. The acceptance of color illustrations is at the discretion of the editor. Costs of color printing for the hard copy publication will be incurred by the authors.

\section{Assignment of copyright and permissions}

At the time of initial submission, all manuscripts must be accompanied by a properly completed authorship form for all authors. Upon submission, authors will not disseminate of any part of the 
material contained in the manuscript without prior written approval from the editor. Nonobservance of this copyright stipulation may result in rejection of the submission for publication.

Assignment of copyright should be uploaded to the website in order to initiate manuscript processing for peer review. Multiple authors should submit separate versions of the form (all signatures should not be on the same form). Manuscripts will not be processed until all signatures have been received. The assignment of copyright form may be obtained on the JMPT submission website.

\section{Conflict of interest}

At the time of initial submission, all manuscripts must be accompanied by a properly completed conflict of interest form for all authors. The conflict of interest form may be obtained on the JMPT submission website or directly from the ICMJE, www.icmje.org.

\section{Permissions}

All permissions should be submitted at the time of initial manuscript submission. It is the corresponding author's responsibility to secure all permissions and provide these to the JMPT editorial office. Permissions include permission to state names or institutions in the acknowledgements, permissions from models who are identifiable in figures, and permissions from patients of case reports (when applicable), etc. Illustrations or content from other publications (print or electronic) must be submitted with written permission from the copyright holder (and author if required) and must be acknowledged in the manuscript, as delineated by the permission granting publisher. For animal or human subject studies, evidence of board approval should be submitted to the website at the initial time of submission. Please upload a jpeg or pdf scan of the approval/exemption letter to the website. Files should be no bigger than $1 \mathrm{MB}$ each.

\section{Supplemental Digital Files}

Supplemental digital files associated with your manuscript, such as video or data files, may be uploaded at the time of ubmission. For any questions regarding supplemental files, please contact the editor.

\section{Informative Articles}

Human subjects research Letters to the editorTrial registriesHIPAA

Conflict of interest Duplicate publication Rapid reviewReview processes Manuscript submissions Authorship

Copyright (C) 2015 Elsevier Inc. All rights reserved. | Privacy Policy | Terms \& Conditions | About Us | Help \& ContactThe content on this site is intended for health professionals.Advertisements on this site do not constitute a guarantee or endorsement by the journal, Association, or publisher of the quality or value of such product or of the claims made for it by its manufacturer.

\section{QUALIS}

\section{Periódicos}

\begin{tabular}{|c|c|c|c|}
\hline ISSN & Título & Área de Avaliação & Classificação \\
\hline $0161-4754$ & $\begin{array}{l}\text { Journal of Manipulative and } \\
\text { Physiological Therapeutics }\end{array}$ & CIÊNCIAS BIOLÓGICAS II & B3 \\
\hline $0161-4754$ & $\begin{array}{l}\text { Journal of Manipulative and } \\
\text { Physiological Therapeutics }\end{array}$ & EDUCAÇÃO FÍSICA & A2 \\
\hline $0161-4754$ & Journal of Manipulative and & ENGENHARIAS III & B1 \\
\hline
\end{tabular}


ISSN Título

Área de Avaliação

Classificação

\begin{tabular}{|l|l|l|l|}
\hline & Physiological Therapeutics & \\
\hline $0161-4754$ & $\begin{array}{l}\text { Journal of Manipulative and } \\
\text { Physiological Therapeutics }\end{array}$ & MEDICINA II & B2 \\
\hline $0161-4754$ & $\begin{array}{l}\text { Journal of Manipulative and } \\
\text { Physiological Therapeutics }\end{array}$ & SAÚDE COLETIVA & B1 \\
\hline
\end{tabular}


ANEXO 8 - COMPROVANTE DE SUBMISSÃO DO MANUSCRITO 3

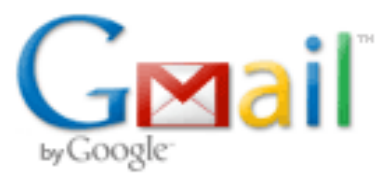

fellipe amatuzzi Teixeira <famatuzzi@gmail.com>

Submission Confirmation Journal of Manipulative and Physiological Therapeutics <cjohnson@nuhs.edu> 10 de novembro de 2015 01:14

Para: famatuzzi@gmail.com, famatuzzi@unb.br Dear FELLIPE,

Your submission entitled "DOES CERVICAL OSTEOPATHIC MANIPULATION TREATMENT PRODUCES IMMEDIATE AND TIME COURSE CARDIOVASCULAR EFFECTS IN HEALTHY INDIVIDUALS?" has been received by Journal of Manipulative and Physiological Therapeutics

You may check on the progress of your paper by logging on to the Elsevier Editorial System as an author. The URL is http://ees.elsevier.com/jmpt/.

Your username is: famatuzzi@gmail.com If you need to retrieve password details, please go to:

http://ees.elsevier.com/jmpt/automail_query.asp

Your manuscript will be given a reference number once an Editor has been assigned. Thank you for submitting your work to this journal. Kind regards, Elsevier Editorial System

Journal of Manipulative and Physiological Therapeutics 
fellipe amatuzzi Teixeira <famatuzzi@gmail.com>

\section{A manuscript number has been assigned: JMPT-D-15-00146}

Journal of Manipulative and Physiological

Therapeutics<cjohnson@nuhs.edu>

Para: famatuzzi@gmail.com, famatuzzi@unb.br
3 de dezembro de $201522: 35$

Ms. Ref. No.: JMPT-D-15-00146

Title: DOES CERVICAL OSTEOPATHIC MANIPULATION TREATMENT

PRODUCES IMMEDIATE AND TIME COURSE CARDIOVASCULAR EFFECTS IN HEALTHY INDIVIDUALS?

Journal of Manipulative and Physiological Therapeutics

Dear FELLIPE,

Your submission entitled "DOES CERVICAL OSTEOPATHIC MANIPULATION TREATMENT PRODUCES IMMEDIATE AND TIME COURSE

CARDIOVASCULAR EFFECTS IN HEALTHY INDIVIDUALS?" has been

assigned the following manuscript number: JMPT-D-15-00146.

You may check on the progress of your paper by logging on to the Elsevier

Editorial System as an author. The URL is http://ees.elsevier.com/jmpt/.

Your username is: famatuzzi@gmail.com

If you need to retrieve password details,

please go to: http://ees.elsevier.com/jmpt/automail_query.asp

Thank you for submitting your work to this journal.

Kind regards,

Claire Johnson, MSEd, DC

Editor-in-Chief

Journal of Manipulative and Physiological Therapeutics

cjohnson@nuhs.edu 